\title{
Kerbgrundkonzepte für die schwingfeste Auslegung von Aluminiumschweißverbindungen am Beispiel der naturharten Legierung AlMg4,5Mn (AW-5083) und der warmausgehärteten Legierung AlMgSi1 T6 (AW-6082 T6)
}

\author{
Vom Fachbereich Maschinenbau \\ an der Technische Universität Darmstadt \\ zur \\ Erlangung des Grades Doktor der Ingenieurwissenschaft (Dr.-Ing.) \\ genehmigte \\ Dissertation \\ vorgelegt von \\ Dipl.-Ing. Christoph Morgenstern \\ aus Frankfurt
}

Berichterstatter:

Mitberichterstatter:

Mitberichterstatter:

Tag der Einreichung:

Tag der mündlichen Prüfung:
Prof. Dr.-Ing. Holger Hanselka

Prof. Dr.-Ing. Timm Seeger

Prof. Dr.-Ing. Cetin Morris Sonsino

12. Januar 2006

17. Mai 2006

Darmstadt 2006

D 17 


\section{Eidesstattliche Erklärung}

Ich versichere an Eides statt, dass ich die vorliegende Arbeit, mit Ausnahme der genannten Hilfen, selbständig angefertigt habe.

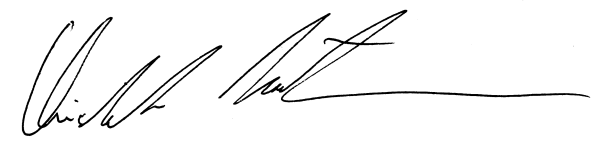

Christoph Morgenstern

Darmstadt, den 11. Januar 2006 


\section{Danksagung}

Die vorliegende Arbeit entstand während meiner Tätigkeit als wissenschaftlicher Mitarbeiter am Fraunhofer-Institut für Betriebsfestigkeit und Systemzuverlässigkeit LBF in Darmstadt. Herrn Prof. Dr.-Ing. Holger Hanselka danke ich sehr für die Unterstützung zur Durchführung dieser Arbeit am LBF.

Herrn Prof. Dr.-Ing. Cetin Morris Sonsino danke ich für stete Förderung, Betreuung und Unterstützung bei meiner Arbeit als wissenschaftlicher Mitarbeiter. Während zahlreicher Diskussionen und mit Hilfe vieler wertvoller Hinweise entstand die Basis für diese Arbeit.

Bei Herrn Prof. Dr.-Ing. Timm Seeger, dem emeritierten Leiter des Fachgebietes Werkstoffmechanik am Institut für Stahlbau und Werkstoffmechanik der TU Darmstadt, bedanke ich mich für die Übernahme des Koreferates und die damit verbundenen Mühen.

Ein besonderer Dank gilt allen Mitarbeitern des Fraunhofer-Instituts für Betriebsfestigkeit und Systemzuverlässigkeit LBF, die mich bei der Durchführung der Untersuchung unterstützt haben und mit zahlreichen Diskussionen und Hinweisen sowie tatkräftiger Hilfe bei den experimentellen Arbeiten zum Gelingen beigetragen haben. Besonders möchte ich dabei die Hilfe durch die Herren Klaus Amelung, Helmut Kohlbacher, Gerhard Hock, Kurt Bayer, Milan Bühn und Horst Matthäus hervorheben.

Für die Unterstützung bei der Modellierung der Schweißnahtgeometrie und Berechnung der Kerbfaktoren mit Hilfe der Finite-Elemente-Methode danke ich Herrn Dipl.-Ing. Francesco Sorbo, ehemals Universität Pisa jetzt GE Oil \& Gas Nuovo Pignone. Herrn Prof. Dr.-Ing. Friedrich Ostermann von der Universität Paderborn danke ich für die Hilfe im Zusammenhang mit der Auswertung und Interpretation der dehnungsgeregelten Versuche.

Ein wesentlicher Teil der Untersuchung war Gegenstand eines von der Forschungsvereinigung Schweißen und Schneiden e.V. des Deutschen Verbandes für Schweißtechnik (DVS) e.V., Düsseldorf, mit Förderung der Arbeitsgemeinschaft für industrielle Forschung (AiF), Köln, durchgeführten Forschungsprojektes „Ermittlung von Grundlagen für die praktische Anwendung örtlicher Konzepte zur Schwingfestigkeitsbewertung geschweißter Aluminiumbauteile". Fachlich betreut wurde das Projekt vom Fachausschuss 9 "Berechnung und Konstruktion". Für die Förderung und Unterstützung sei gedankt. Ein weiterer Dank gilt den Mitgliedern des projektbegleitenden Betreuerkreises der Industrie unter Leitung von Herrn Dr.-Ing. Manfred Kaßner, Alstom GmbH, Salzgitter, die mit fachlichem Rat zur Seite standen. Ebenfalls sei dem Bundesministerium für Bildung und Forschung für die finanzielle Unterstützung des Projektes "Grundlagen für den Leichtbau energiesparender Nutzfahrzeuge auf Basis neuartiger Schweißverbindungen und Auslegungsverfahren für Aluminiumkonstruktionen", sowie dem projektbegleitenden Ausschuss unter der Leitung von Dipl.-Ing. Manfred Streicher, MAN Nutzfahrzeuge, München, gedankt, dessen Ergebnisse und Anregungen zum Teil in diese Arbeit einflossen.

Herrn Dr.-Ing. Jacek Grzesiuk, ehemals IMAB Clausthal, jetzt Robert Bosch GmbH, Schwieberdingen, danke ich dafür, dass er mir die Ergebnisse zu den HY- Nähten aus seiner Dissertation „Einfluss der Nahtvorbereitung und Nahtausführung auf die Schwingfestigkeit hochwertiger Aluminiumkonstruktionen" für diese Arbeit zur Verfügung stellte.

Ebenfalls sei ein Dank ausgesprochen an Herrn Dipl.-Ing. Jürgen Kotowski und Herrn Dr.-Ing. Thomas Nitschke-Pagel vom Institut für Schweißtechnik (ifs) der TU Braunschweig für die Durchührung einer großen Zahl an Schwingfestigkeitsversuchen mit Aluminiumschweißverbindungen, sowie Härtemessungen. 


\section{Summary}

For an application of aluminium welded joints in the manufacturing of passenger cars, commercial vehicles, plant constructions and railway carriages, the objective was to evaluate existing design concepts for a fatigue life assessment under constant and variable amplitude loadings.

Fatigue tests were carried out with $5 \mathrm{~mm}$ thick aluminium welded joints of the artificially aged wrought aluminium alloy AW- 6082 T6 (AIMgSi1 T6) with different notch sizes ( $\mathrm{K}_{\mathrm{t}, \mathrm{a}}=1.3$ to 7.5). The experimental results were compared with former test results by the naturally aged wrought aluminium alloy AW-5083 (AlMg4.5Mn) and further alloys.

For a comprehensive material characterisation strain controlled fatigue tests with the parent metal, the weld metal and the heat-affected zone with unnotched flat specimens for determining the elastic-plastic material behaviour were carried out. The cyclic material behaviour under fully reversed and under pulsating loadings with unnotched and crack-like notched flat specimens of the three material conditions were obtained from stress controlled fatigue test. In addition, the fracture mechanics values were derived from crack growth tests.

The microstructure was documented with metallographic analyses. Linear and two dimensional hardness distribution of the welded joints and etched sections were carried out. They show a milling texture of the parent metal and the cast-like microstructure of the weld metal and explain the different fatigue behaviour depending on the material conditions.

With a uniform evaluation from new and former results of fatigue strength tests with different notched flat specimens of parent metal, weld metal and heat-affected zone the substitute structural length $\rho^{\star}$ according to Radaj was derived. The submitted values were between $\rho^{*}=$ 0.22 to 0.54 and approximately match the values on the literature.

For the application of the micro-support concept the appropriated substitute structural length $\rho^{*}$ was used for the respective material in which the fatigue cracks are initiated at the weld. For the butt welded joints without internal gap it is the constant of the heat affected zone respectively the parent metal and for the butt welded joints with internal root gap it is the constant of the weld metal.

The application of the micro-support concept with the substitute structural length $\rho^{\star}$ according to Radaj derived form experimental fatigue strength tests resulted in correct fatigue life estimations under fully reversed and pulsating loadings. The effort for sourcing the experimental data for this concept is considerable high. Abstracting from the existing concept which depends on the material and microstructure a more universal concept was developed.

The local stress concept derived in this investigation using the fictitious notch radius of $r_{f}=1.0$ $\mathrm{mm}$ can be applied to aluminium welded joints from plates with thickness of $t>5 \mathrm{~mm}$ and complies with the demand for a simple uniform concept is suitable to use in modern fatigue design recommendations.

For the verification of the applicability of the local stress concept with the fictitious notch radius of $r_{f}=1.0 \mathrm{~mm}$ fatigue strength test with aluminium welded joints with thickness $t=5,8$ and $25 \mathrm{~mm}$ were carried out covering the application range of aluminium sheet metal. The weld geometries ranges from butt welds without root gap and little seam height and transversal stiffeners (low notch factor) to butt welds with strong seam height (single side welded butt welds (with intermediated notch factor) up to butt welds with internal root gap and $\mathrm{H}$-shape specimen with 
fillet welds (high notch factor) and covers a wide range of technical relevant notches and weld geometries. Three different aluminium alloys AW-5083 (AlMg4,5Mn), AW-6082 T6 (AlMgSi1 T6) and AW-6005A T6 (AlMgSi0,7 T6) in this concept verification investigation covers a large range in this parameter field.

With this investigation it could be showed that the local stress concept using the fictitious notch radius of $r_{f}=1.0 \mathrm{~mm}$ can be applied to aluminium welded joints from plates with thickness of $t=$ 5 to $25 \mathrm{~mm}$ independently from the alloy (AlMg4.5Mn or AlMgSi1 T6) and weld geometries (fully or partially penetrated butt welds, transversal stiffeners). For the better application of the concept additionally a proposal for IIW-Fatigue Design Recommendations was made. 


\section{Inhalt}

0 Zusammenfassung 3

$1 \quad$ Einleitung 5

2 Stand der Technik und Wissenschaft zur schwingfesten Auslegung von $\begin{array}{lr}\text { Schweißverbindungen aus Aluminium } & \mathbf{8}\end{array}$

$2.1 \quad$ Nennspannungskonzept 9

2.2 Strukturbeanspruchungskonzept 10

2.2.1 Strukturspannungskonzept nach IIW-Empfehlung - Grobbleche 12

2.2.2 Strukturspannungskonzept nach Dong 13

2.2.3 Strukturspannungskonzept nach Xiao und Yamada 15

2.2.4 Strukturspannungskonzept nach Fermer und Svensson - Feinbleche 17

2.2.5 Hot-Spot-Strukturspannungskonzept nach Dijkstra und de Back 18

2.2.6 Strukturdehnungskonzept nach Haibach 19

2.3 Kerbbeanspruchungskonzepte 19

2.3.1 Kerbdehnungskonzept 19

2.3.2 Kerbspannungskonzept nach Radaj 23

2.3.3 Bestimmung der Ersatzstrukturlänge nach Neuber 24

2.3.4 Bestimmung der Ersatzstrukturlänge nach Radaj 26

2.3.5 Kerbgrundkonzept mit fiktivem Ersatzradius nach Seeger 26

2.4 Werkstoffvolumenkonzept 28

2.5 Bruchmechanisches Konzept 29

2.5.1 Rißfortschrittskonzept 29

2.5.2 Spannungsintensitätskonzept 32

$2.6 \quad$ Schadensparameter 34

2.7 Lebensdauerabschätzung bei veränderlichen Betriebsbelastungen 35

2.8 Ansätze für die vorliegende Arbeit 39

$3 \quad$ Werkstoffe, Proben und Versuchsdurchführung 41

3.1 Werkstoffe und Proben 41

3.1.1 Probenform und Fertigung $\quad 42$

3.1.2 Härtemessungen 47

3.1.3 Gefügebetrachtungen 52

3.1.4 Geometrische Beschreibung der Schweißnähte und Berechnung der Formzahlen 58

3.2 Versuchstechniken 62

3.2.1 Versuchsdurchführung 62

3.2.2 Erfassung des Anrisses und Rissfortschrittverhaltens 64

$4 \quad$ Schwingfestigkeitsuntersuchungen $\quad 67$

4.1 Schwingfestigkeit von Grundwerkstoff, Schweißgut und Wärmeeinflusszone 67

4.1.1 Elastisch-plastisches Materialverhalten im ungekerbten Zustand unter $\begin{array}{ll}\text { Dehnungssteuerung } & 67\end{array}$

4.1.2 Schwingfestigkeit im ungekerbten und gekerbten Zustand unter Lastregelung 73

4.1.3 Berechnung der Ersatzstrukturlänge 78

4.1.4 Vergleich der ermittelten Ersatzstrukturlänge mit Angaben aus der Literatur 81

4.2 Rissfortschrittsverhalten - Ermittlung bruchmechanischer Kennwerte 82

4.3 Schwingfestigkeitsverhalten von Schweißverbindungen unter Laststeuerung 86 
$5 \quad$ Anwendung von Kerbspannungskonzepten zur Berechnung der Schwingfestigkeit von Schweißverbindungen

5.1 Konzept der Mikrostützwirkung

5.2 Konzept des fiktiven Ersatzradius 95

$6 \quad$ Vorschlag für die IIW-Richtlinie $\quad 104$

$7 \quad$ Schlussfolgerungen und Ausblick 112

$8 \quad$ Literatur 114

$9 \quad$ Abkürzungen und Formelzeichen $\quad 129$

Anhang A: Schweißprotokolle - Tabelle A1 - A7

Anhang B: Schwingfestigkeitsergebnisse - Tabelle B1 - B19

Anhang C: Rissfortschrittkurven - Bild C1 - C6 


\section{$0 \quad$ Zusammenfassung}

Für die Anwendung von Aluminiumschweißverbindungen im Personenwagen-, Nutzfahrzeug-, Anlagen und Waggonbau wurden vorhandene lokale Bemessungskonzepte für eine Lebensdauerabschätzung unter konstanten und variablen Amplituden unter verschiedenen Gesichtspunkten überprüft und weiterentwickelt. .

Dazu wurde zunächst das Schwingfestigkeitsverhalten von $5 \mathrm{~mm}$ dicken Aluminiumschweißverbindungen der warmausgehärteten Legierung AlMgSi1 T6 (AW-6082) verschiedener Kerbschärfen $\left(K_{t, a}=1,3\right.$ bis 7,5$)$ experimentell untersucht werden. Die Ergebnisse wurden mit bereits vorliegenden Versuchen an Verbindungen aus der naturharten Legierung AlMg4,5Mn (AW-5083) und weiterer Aluminiumlegierungen verglichen.

Für eine ausführliche Werkstoffcharakterisierung wurden dehnungsgeregelte Schwingfestigkeitsversuche am Grundwerkstoff, dem Schweißgut und der Wärmeeinflusszone zur Bestimmung des elastisch-plastischen Werkstoffverhaltens an ungekerbten Flachproben durchgeführt. Darüber hinaus wurde mit kraftgeregelten Schwingfestigkeitsversuchen mit ungekerbten und rissartig scharf gekerbten Flachproben unter wechselnder und schwellender Beanspruchung für die drei Werkstoffzustände das zyklische Verhalten bestimmt. Ergänzend wurden mit Rissfortschrittversuchen die bruchmechanischen Kennwerte ermittelt.

Begleitend wurden metallographische Untersuchungen zur Gefügedokumentation durchgeführt. Neben der flächigen und linearen Mikrohärtemessung an den Schweißverbindungen, wurden geätzte Schliffe zur Gefügedarstellung angefertigt. Diese zeigen beim Grundwerkstoff eine geordnete Kornausrichtung (Walztextur), während im Schweißgut ein gussähnliches Gefüge vorliegt. Dies erklärt das unterschiedliche Schwingfestigkeitsverhalten in Abhängigkeit vom Werkstoffzustand.

Aus den neuen und bereits vorhandenen Ergebnissen von Schwingfestigkeitsversuchen verschieden gekerbter Flachproben an Grundwerkstoff, Schweißgut und Wärmeeinflusszone wurde durch gemeinsame, einheitliche Auswertung die Ersatzstrukturlänge $\rho^{*}$ nach Radaj abgeleitet. Es ergaben sich Werte für $\rho^{*} z$ wischen 0,22 und 0,54. Die ermittelten Werte stimmen näherungsweise mit Angaben aus der Literatur überein.

Für die Anwendung des Mikrostützwirkungskonzeptes wurden die an der Versagensstellen der Schweißverbindung relevanten Kennwerte zugrunde gelegt. Dies sind für Verbindungen mit Wurzelspalt das Schweißgut und für Verbindungen ohne Wurzelspalt die Wärmeeinflusszone bzw. der Grundwerkstoff.

Die Anwendung des Konzeptes der Mikrostützwirkung mit den experimentell ermittelten und nach Radaj ausgewerteten Daten zur Ersatzstrukturlänge $\rho^{*}$ bei der Lebensdauerabschätzung unter konstanten Amplituden führt zu zutreffenden Ergebnissen. Jedoch ist der Aufwand zur Gewinnung der Eingangsdaten erheblich. Durch Abstraktion von werkstoff- und gefügespezifischen Kenngrößen konnte ein deutlich universelles örtliches Konzept entwickelt werden.

Das in dieser Arbeit für die Anwendung bei Aluminiumschweißverbindung ab einer Dicke von $t>$ $5 \mathrm{~mm}$ abgeleitete örtliche Konzept des fiktiven Ersatzradius mit $r_{f}=1,0 \mathrm{~mm}$ erfüllt diese 
Forderungen und ist darüber hinaus geeignet, mit entsprechender Aufbereitung Eingang in technische Regelwerke zu finden.

Für den Nachweis der Anwendbarkeit des Konzeptes des fiktiven Ersatzradius mit $r_{f}=1,0 \mathrm{~mm}$ wurden Schwingfestigkeitsversuche mit Schweißverbindungen in der Dicke $t=5,8$ und $25 \mathrm{~mm}$ durchgeführt, die den Anwendungsbereich der Aluminiumgrobbleche repräsentieren. Die in der Untersuchung mit einbezogenen Schweißnahtgeometrien deckten von Stumpfstößen ohne Wurzelspalt mit geringer Nahtüberhöhung bzw. Quersteifen (kleine Kerbformzahl) über Stumpfstoßverbindungen mit starker Nahtüberhöhung (einseitig geschweißte Stumpfstöße; mittlere Kerbformzahl) bis zu Stumpfstößen mit innerem Wurzelspalt und H-Proben mit Kehlnähten (sehr große Kerbformzahl) einen weiten Bereich praxisrelevanter Kerbfälle und Geometrien ab. Die Berücksichtigung von drei verschiedenen Aluminiumlegierungen (AlMg4,5Mn (AW-5083), AlMgSi1 T6 (AW-6082 T6) und AlMgSi0,7 T6 (AW-6005A T6)) bei der Konzeptüberprüfung deckt auch in diesem Bereich ein großes Feld möglicher Parameter ab. Mit dieser Untersuchung kann dem Anwender die Eignung des örtlichen Spannungskonzeptes mit einem fiktivem Ersatzradius von $r_{f}=1,0 \mathrm{~mm}$ für den Werkstoffdickenbereich $\mathrm{t} \geq 5$ bis $25 \mathrm{~mm}$ für die Festigkeitsbeurteilung von Aluminiumschweißverbindungen aufgezeigt werden. Darüber hinaus wurde zur besseren praktischen Handhabbarkeit in der industriellen Praxis ein Vorschlag für eine IIW-Empfehlung erarbeitet. 


\section{$1 \quad$ Einleitung}

Lokale Kerbspannungs- und Kerbspannungsintensitätskonzepte gewinnen neben dem verbreiteten globalen Nennspannungskonzept zunehmend an Bedeutung für die rechnerische Auslegung von Schweißkonstruktionen.

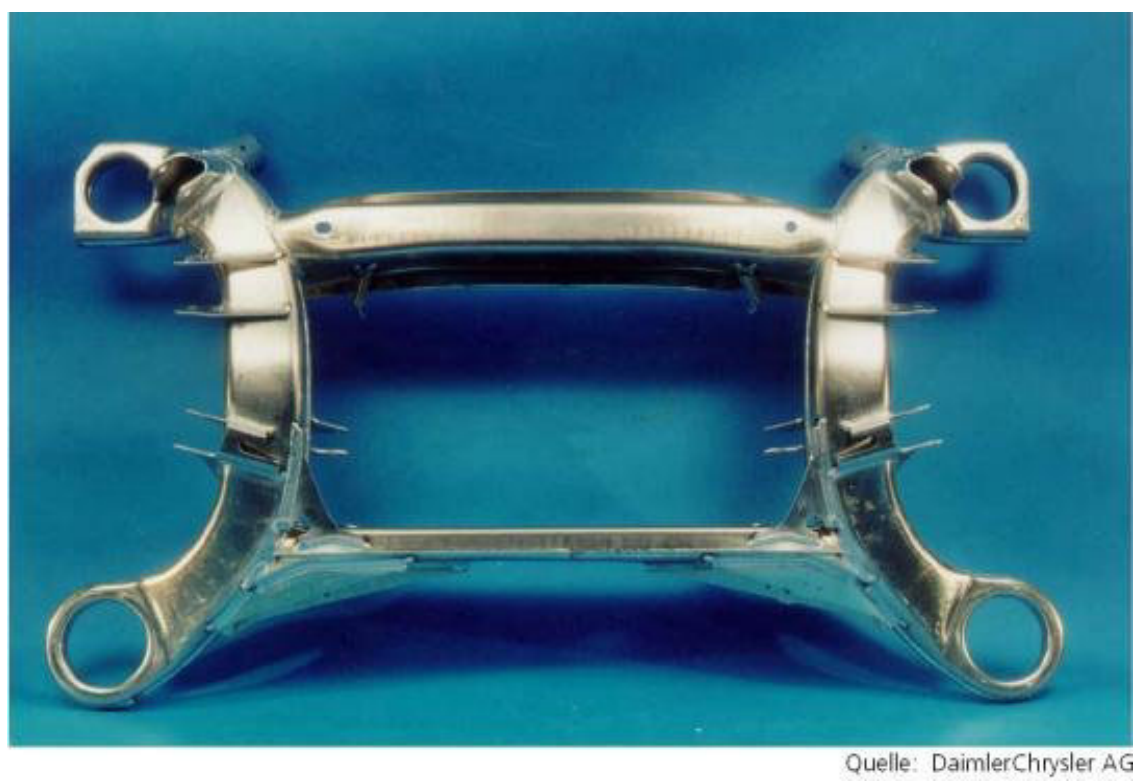

Bild 1.1: Geschweißter Aluminium-Hinterachsträger der Mercedes S-Klasse

Die Abschätzung der Schwingfestigkeit für den Bereich hoher Schwingspielzahlen $\left(N>10^{6}\right)$ von Nahtschweißverbindungen, ausgehend von den Kerbspannungen an der Nahtübergangs- und Nahtwurzelkerbe, (elastische und elastisch-plastische "Kerbgrundkonzept") wurde in den vergangenen Jahren für Stahlwerkstoffe vielfach untersucht. Das so genannte Kerbgrundkonzept wird inzwischen als ein regelwerkfähiges und industriell anwendbares Verfahren des rechnerischen Festigkeitsnachweises sowie der Konstruktions- und Fertigungsoptimierung empfohlen. Der Grundgedanke des Verfahrens [Rad84, Rad85-1, Rad85-2, Rad88-1, Rad90, Rad95, Rad95-2, Rad98-1, Rad98-2] besteht darin, die Nahtübergangskerben zur Erfassung der Mikrostützwirkung nach Neuber fiktiv zu runden und die sich ergebenden Kerbspannungen mit der lokalen Ermüdungsfestigkeit am Kerbgrund zu vergleichen. Diese Vorgehensweise soll in dieser Arbeit nicht nur auf die Anwendbarkeit auf Aluminiumschweißverbindungen im Bereich hoher Schwingspielzahlen überprüft, sondern auf den Bereich der Zeit- $\left(N \approx 5 * 10^{4}-10^{6}\right)$ und Kurzzeitschwingfestigkeit $\left(\mathrm{N}<5^{*} 10^{4}\right)$ ausgedehnt werden. Weiter soll der Legierungseinfluss untersucht werden. 


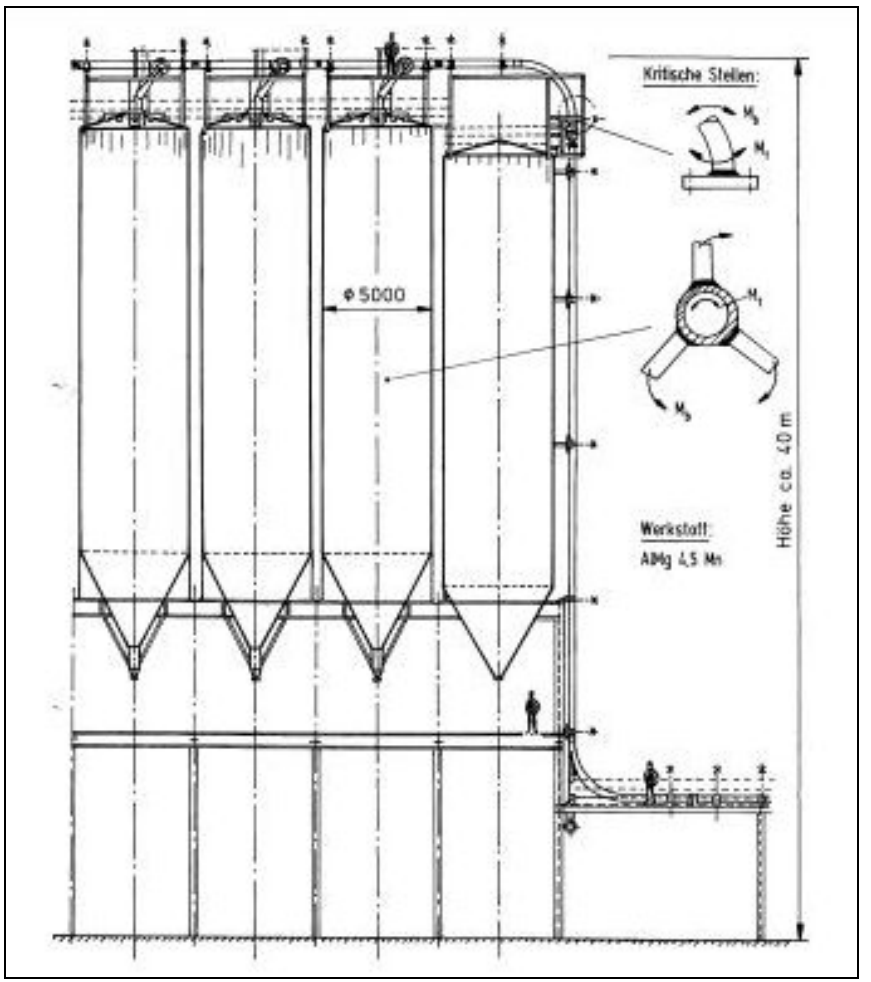

Bild 1.2: Silos aus Aluminium zur Aufbewahrung von Kunststoffgranulaten

Die Anwendung der lokalen Konzepte soll zunächst anhand der Lebensdauerberechnung unter konstanten Amplituden überprüft und weiterentwickelt werden.

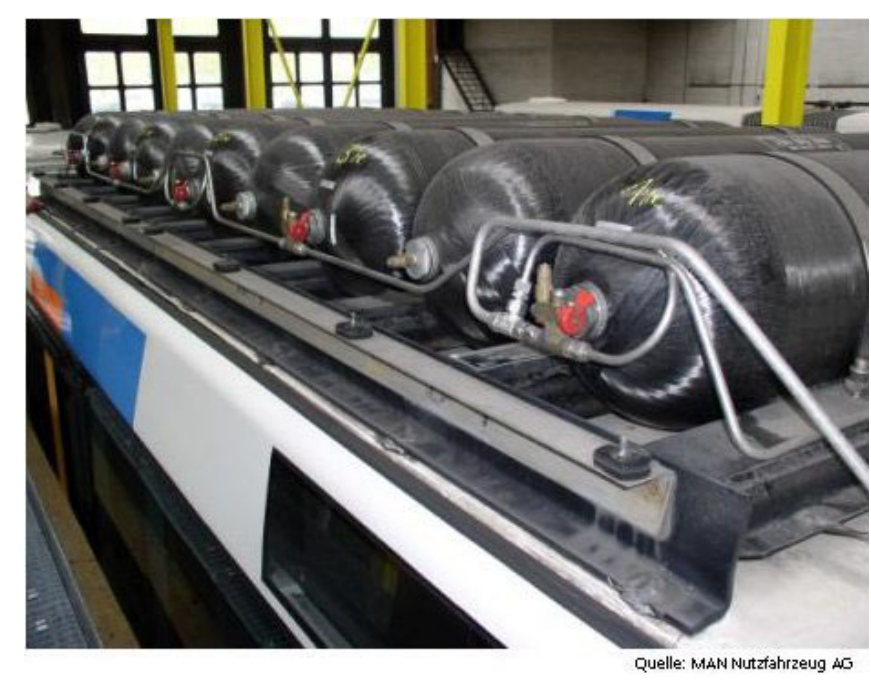

Bild 1.3: Halterung aus Aluminium von Druckbehältern auf einem Busdach

Bei den untersuchten Schweißverbindungen handelt es sich um Stumpfstöße ohne Wurzelspalt, welche eine relativ niedrige Formzahl aufweisen, und um Stumpfstöße mit Wurzelspalt, bei denen am Wurzelspalt eine hohe Spannungskonzentration vorliegt. Dadurch wird ein weiter Bereich von Spannungskonzentrationen untersucht. Im Hinblick auf eine Anwendung der Schweißverbindungen im Personenwagen-, Nutzfahrzeug-, Waggon- und Anlagenbau, Bild 1.1 bis 1.5 wurden Versuchskörper in den Dicken 5 und $25 \mathrm{~mm}$ gewählt. Ebenso wurde ein breites Anwendungsfeld durch die untersuchten Werkstoffe abgedeckt. 


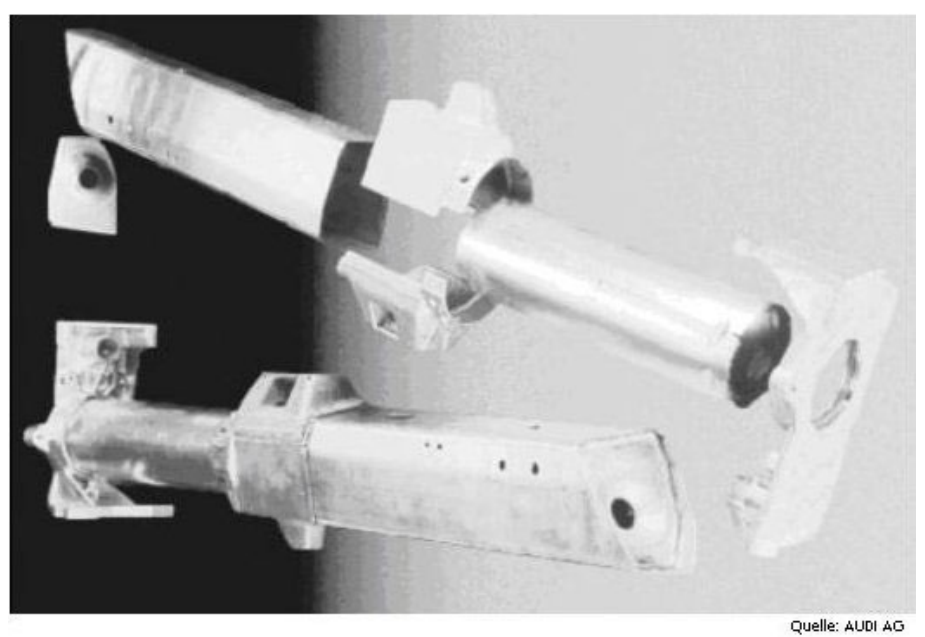

Bild 1.4: Komponenten eines Space-Frame-Längsträgers aus Aluminium

In Abhängigkeit von der Formzahl unterteilt sich die Lebensdauer in Entstehung eines technischen Anrisses mit definierter Tiefe ( z.B. $a=0,25 \mathrm{~mm}$ ) und Risswachstum. Die erforderlichen Werkstoffkenndaten der verwendeten Aluminiumlegierungen AlMgSi1 T6 (AW-6082) und AlMg4,5Mn (AW-5083) werden teilweise experimentell ermittelt oder durch Auswertung vorhandener Versuchsergebnisse aus vorangegangenen Untersuchungen gewonnen. Dabei soll nicht nur das Schwingfestigkeitsverhalten des Grundwerkstoffs, sondern auch des Schweißgutes und der Wärmeeinflusszone untersucht werden.

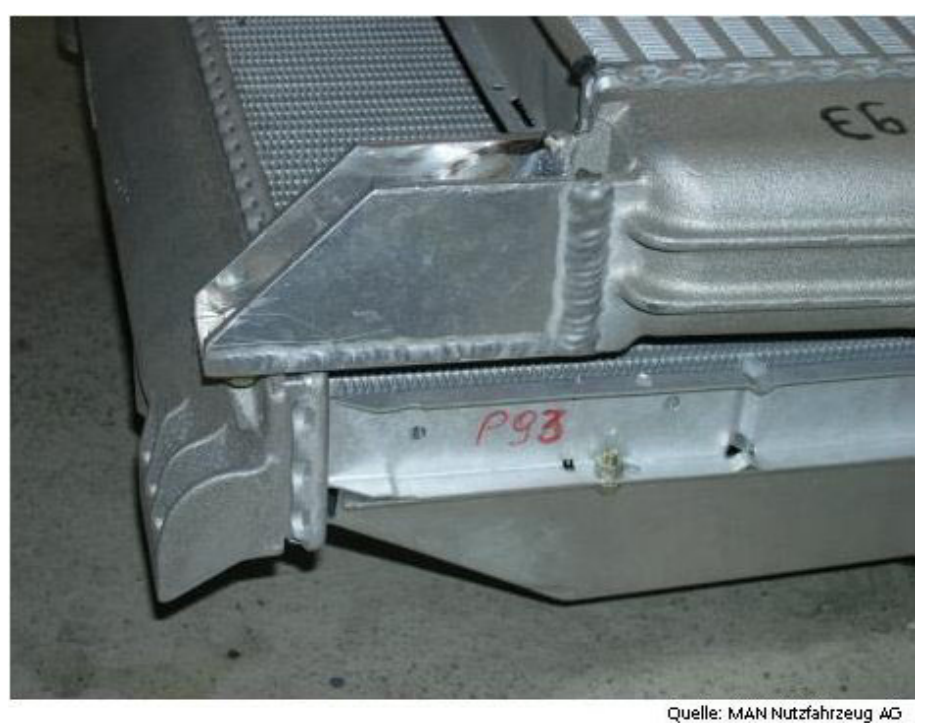

Bild 1.5: Geschweißte Halterung eines Kühlers aus Aluminium

Das Ziel der vorliegenden Arbeit ist es, ausgehend vom jetzigen Stand der Technik und Wissenschaft ein Bemessungsverfahren zur Lebensdauerabschätzung von Aluminiumschweißverbindungen zu entwickeln, welches nicht nur im Bereich hoher Schwingspielzahlen, sondern auch im Bereich der Zeitschwingfestigkeit gültig ist und möglichst unabhängig von der Legierung und dem lokalen Gefüge ist. 


\section{Stand der Technik und Wissenschaft zur schwingfesten Auslegung von Schweißverbindungen aus Aluminium}

Für die Festigkeitsbewertung von geschweißten Bauteilen sind verschiedene Nachweiskonzepte, Bild 2.1, entwickelt worden, die in Abhängigkeit vom gewählten Konzept eine unterschiedliche ortsbezogene Einschätzung der Beanspruchungssituation von Schweißverbindungen erlauben [Son05-3, Kas04].

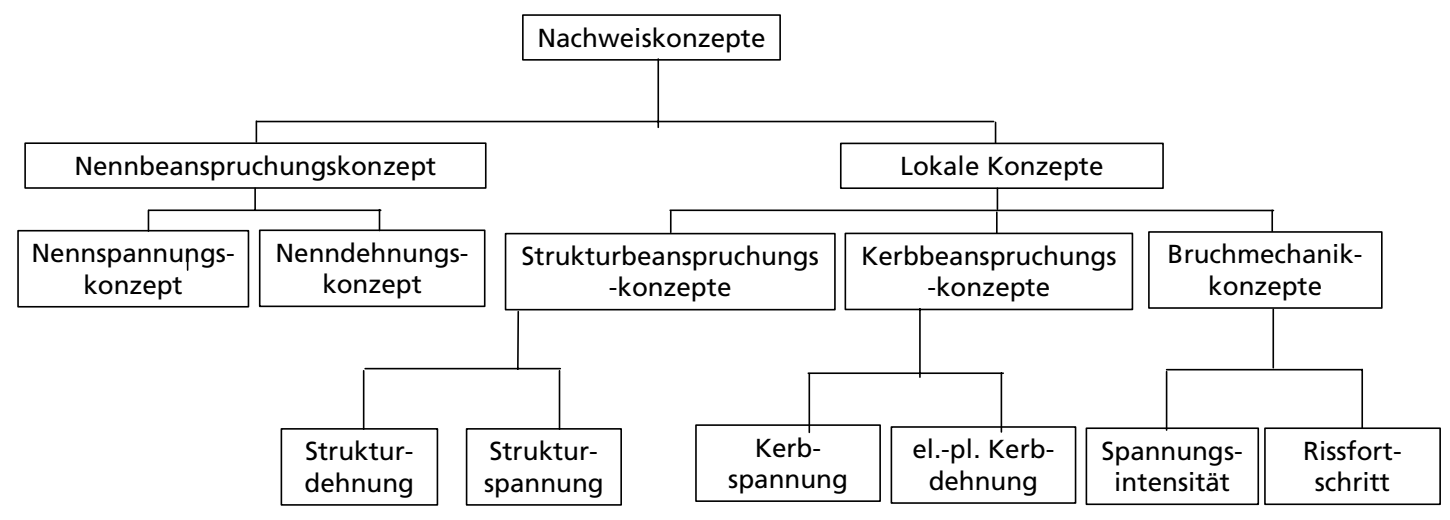

Bild 2.1: Nachweiskonzepte für die schwingfeste Auslegung geschweißter Bauteile

Aufgrund der eingeschränkten Anwendbarkeit des Nennspannungskonzeptes, das nach wie vor fester Bestandteil von verschiedenen Regelwerken z.B. [Eur92, Eur98, Bri90, Rad90, Hob03] ist, sind mit dem Struktur- und Kerbspannungskonzept [See96, Rad98-2, Rad00, Hob03] und z. T. mit dem Bruchmechanikkonzept [FKM02] Nachweisverfahren entstanden, die eine deutlich detailgenauere Festigkeitsanalyse von geschweißten Bauteilen ermöglichen. So läßt sich mit dem Strukturspannungskonzept die im Verbindungsbereich vorliegenden Bauteilgeometrie unmittelbar bei der Beanspruchungsermittelung erfassen. Dadurch kann eine wirtschaftlichere und zugleich sichere Bemessung von geschweißten, schwingbelasteten Bauteilen erfolgen. Darüber hinaus gestatten örtliche Konzepte mit den Möglichkeiten numerischer Berechnungsverfahren (z.B. Finite-Element.Methoden), Beanspruchungen in einem deutlich geringeren Abstand zur Versagensstelle (Struktur) oder direkt in der Versagensstelle (Kerbe) zu ermitteln, was mit dem Nennspannungskonzept nicht möglich ist.

Im Folgenden sollen die verschiedenen Konzepte mit ihren Grundprinzipien kurz dargestellt werden. Allerdings werden hier nur Konzepte für Nahtschweißverbindungen besprochen; für Konzepte für Punktschweißverbindungen sei auf die Literatur verwiesen [Rup92, Rad00, Wal03, See05]. 


\subsection{Nennspannungskonzept}

Für die Anwendung des Nennspannungskonzeptes in Regelwerken muss eine Nennspannung definiert und eine Kerbfallklasse festgelegt werden, der eine Wöhlerlinie zugeordnet ist, Bild 2.2.

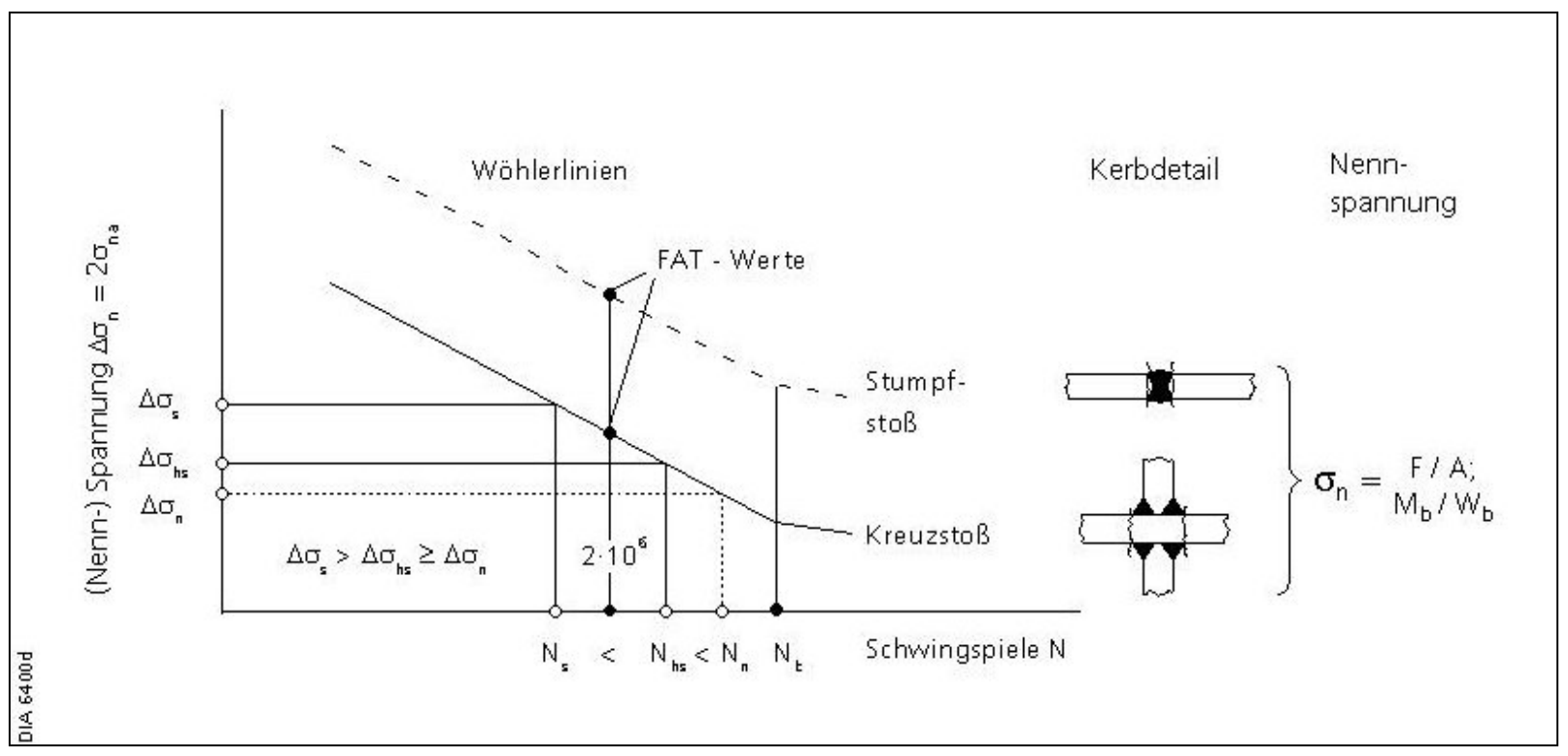

Bild 2.2: Beurteilung der Lebensdauer mit Nennspannungswöhlerlinien

Nachzuweisen ist, dass die Nennspannungen im tragenden Querschnitt an der kritischen Stelle kleiner als die zulässigen Nennspannungen (ertragbare Nennspannungen reduziert um einen Sicherheitsfaktor) sind. Die Ableitung der Bauteilwöhlerlinie setzt eine statistisch abgesicherte Nennspannungswöhlerlinie für den jeweiligen Anwendungsfall (Kerbfall) voraus.

In Abhängigkeit von der vorliegenden Wöhlerlinie bilden Anriss mit definierter Länge und Tiefe oder Bruch das Versagenskriterium.

Das Nennspannungskonzept zeichnet sich durch seinen geringen Aufwand bei einem Festigkeitsnachweis aus. Für Bauteile mit einfacher Geometrie, für die sich Nennspannungen und Kerbfallklassen definieren lassen, ist es die Grundlage der Dimensionierung und ist in Regelwerken verankert [Rad95, Hai89, Klo89, Bux92].

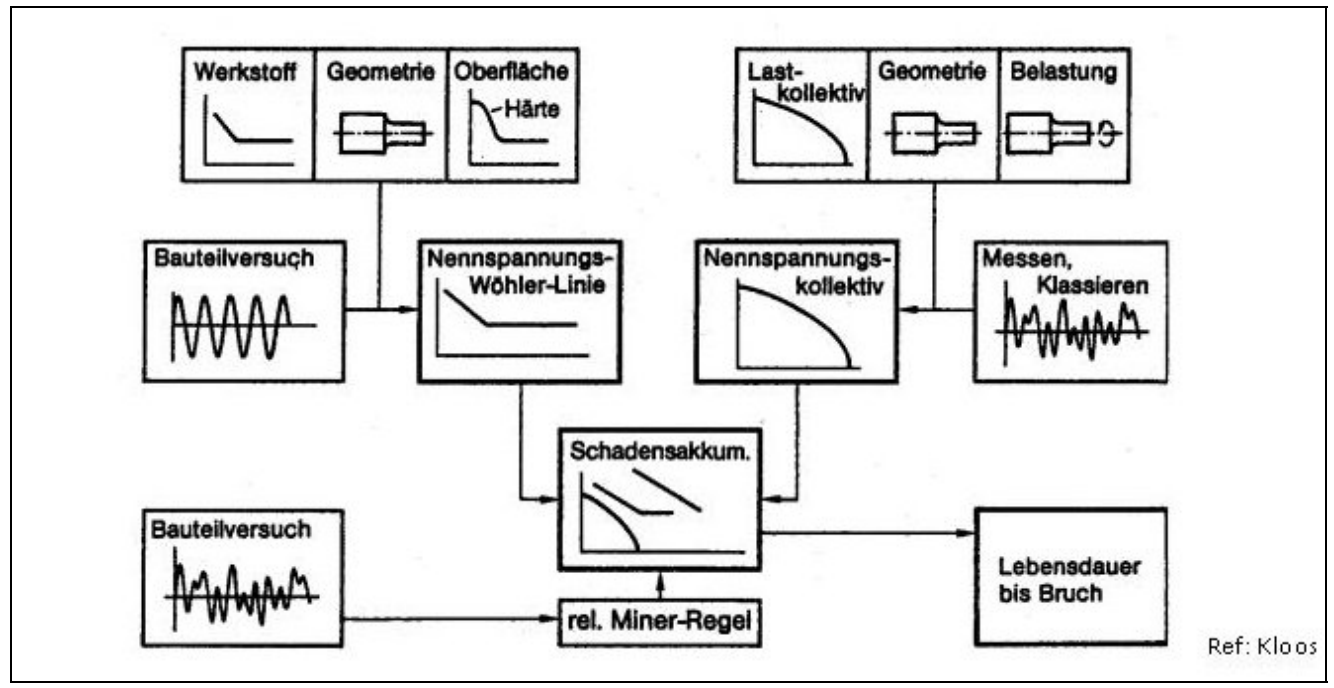

Bild 2.3: Nennspannungskonzept 
Zur Bewertung der Schwingfestigkeit von Schweißverbindungen wird die NennspannungsWöhlerlinie in Abhängigkeit von Werkstoff, Kerbfallklasse (Nahtgeometrie) und Qualitätsklasse (Fertigung) festgelegt. Die Nennspannung wird im allgemeinen für den Querschnitt, der an die Schweißnaht angrenzt, ermittelt. Den Kerbfall- und Qualitätsklassen sind Scharen vereinheitlichter Nennspannungs- Wöhlerlinien zugeordnet. Die zu beurteilende Schweißverbindung wird entsprechend der Form, Nahtart, Belastung und Fertigungsqualität den standardisierten Nennspannungs- Wöhlerlinien gegenübergestellt [Rad98-1, Rad98-2]. In Kombination mit der relativen Miner- Regel in zweckmäßig modifizierter Form ermöglicht das Nennspannungskonzept eine Tendenz- und Relativaussage zur Lebensdauer einer Schweißkonstruktion, Bild 2.3. Ohne experimentell abgesicherte Referenzergebnisse ist jedoch eine Bemessung nicht möglich.

Tabelle 2.1 zeigt beispielhaft zulässige Nennspannungs-Schwingbreiten nach der IIW-Richtlinie [Hob03] für Stahl und Aluminium. Die aufgeführten Werte gelten für $\mathrm{N}=2 * 10^{6}$ Schwingspiele und geben die Schwingfestigkeitsklasse (FAT) an.

\begin{tabular}{|c|c|c|c|c|}
\hline No. & Structural Detail & $\begin{array}{l}\text { Description } \\
\text { (St.= steel; } \mathrm{Al}=\text { aluminium) }\end{array}$ & $\begin{array}{l}\text { FAT } \\
\text { St. }\end{array}$ & $\begin{array}{l}\text { FAT } \\
\text { Al. }\end{array}$ \\
\hline 211 & & $\begin{array}{l}\text { Transverse loaded butt weld } \\
\text { (X-groove or V-groove) ground } \\
\text { flush to plate, } 100 \% \mathrm{NDT}\end{array}$ & 100 & 40 \\
\hline 212 & & $\begin{array}{l}\text { Transwerse butt weld made in shop } \\
\text { in flat position, } \\
\text { toe angle } \leq 30^{\circ} \text {, NDT }\end{array}$ & 90 & 36 \\
\hline 213 & & $\begin{array}{l}\text { Transverse butt weld not satisfying } \\
\text { conditions of } 212 \text {, NDT } \\
\text { A1.: Butt weld with toe angle }<50^{\circ} \\
\quad \text { Butt welds with toe angle }>50^{\circ}\end{array}$ & 80 & $\begin{array}{l}32 \\
25\end{array}$ \\
\hline
\end{tabular}

Tabelle 2.1: Kerbfallklassen (FAT-Werte) nach IIW-Richtlinie (beispielhaft)

\section{$2.2 \quad$ Strukturbeanspruchungskonzept}

Bei komplexen Geometrien, bei denen die Definition einer Nennspannung sowie die Zuordnung einer Kerbfallklasse mit Schwierigkeiten verbunden ist, kann mit den Strukturbeanspruchungskonzept eine genauere Festigkeitsbewertung erfolgen. Hierbei ist, je nach Konzept, die Kenntnis unterschiedlicher Beanspruchungsgrößen erforderlich.

Die ursprüngliche Form des Strukturbeanspruchungskonzepts ist die Strukturdehnungsvariante. Die mit einem DMS am Nahtfuss gemessene Dehnung bzw. die daraus abgeleitete Spannung dient dem Festigkeitsnachweis [Hai02], Bild 2.4. Mit der verbreiteten Anwendung von Dehnmessstreifen und später dem Aufkommen der Finite-Element-Methode wurde die Strukturspannungsvariante bedeutsam, die für Rohrknoten in der Bau- und Offshore-Technik, verbunden mit der Hot-Spot-Spannung, zu einem kodifizierten Nachweisverfahren entwickelt wurde [Hob05, Rad98-2]. Die Übertragung von Rohrknoten auf die plattenartigen Strukturen im Schiffbau und weiterer Anwendungsgebiete erfolgte vornehmlich im Rahmen des IIW [Nie92, Nie95]. 


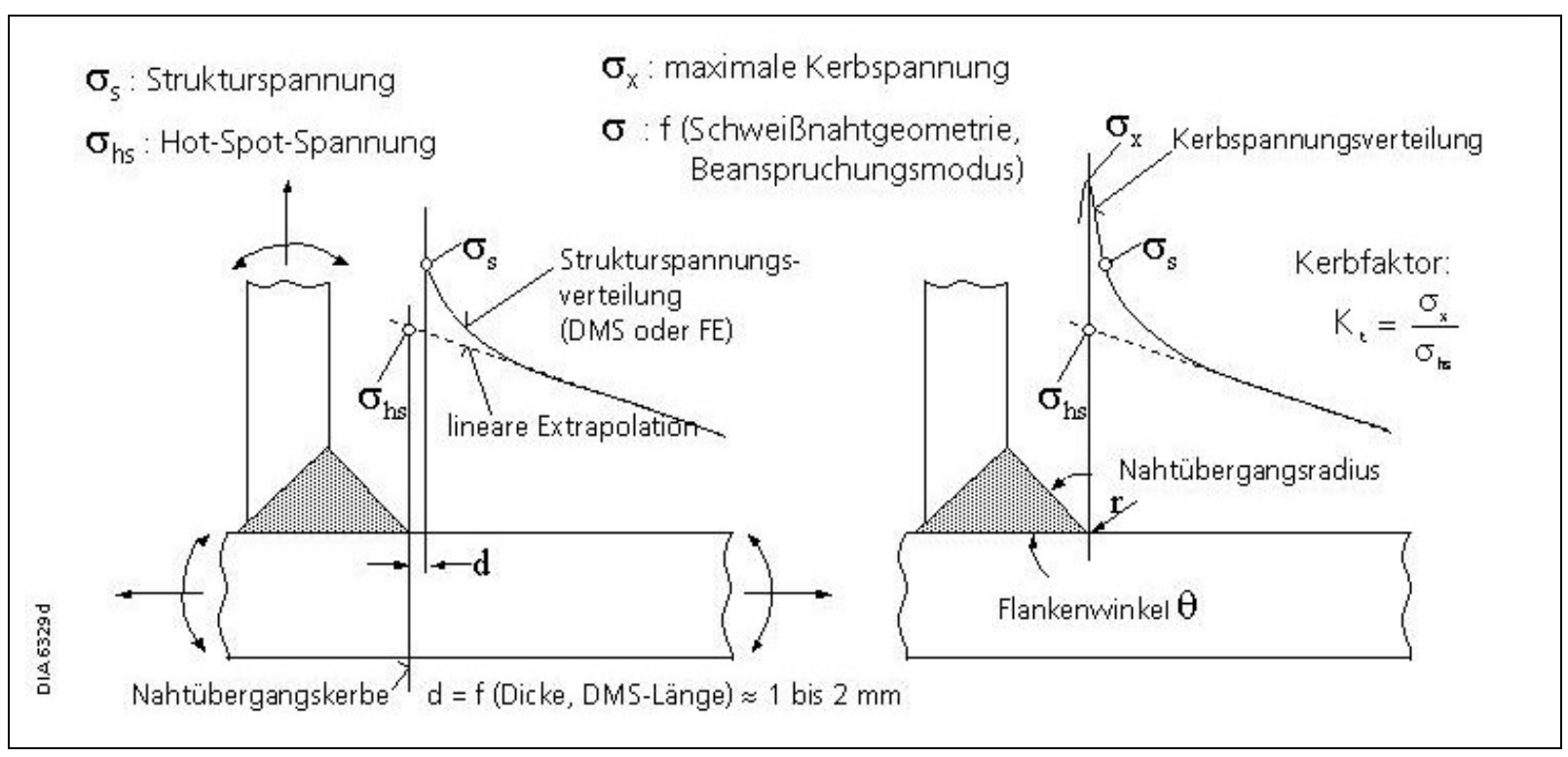

Bild 2.4: Definition der Spannungen für das Strukturspannungskonzept

Die Größe der Strukturbeanspruchung hängt grundsätzlich vor allem von der Form und Anordnung der zu verbindenden Bauteile ab, wobei sich dieser Struktureffekt insbesondere auf die Beanspruchungen quer zur Naht auswirkt. Somit dient das Strukturbeanspruchungskonzept vor allem zur Festigkeitsbewertung der in dieser Richtung auftretenden Beanspruchungen.

Von den Strukturbeanspruchungskonzepten existieren zahlreiche Varianten, von denen hier sowohl Varianten des Strukturspannungs- als auch des Strukturdehnungskonzeptes kurz dargestellt werden sollen:

- Das Strukturspannungskonzept für Grobblechanwendungen, wie es zur Zeit in der IIWRichtlinie kodifiziert ist [Hob05]

- Die Konzepte nach Dong sowie nach Xiao und Yamada, welche mit einer Innenlinearisierung zur Bestimmung der Strukturspannung aus FE-Modellen arbeiten [Don01, Don02, Xia04]

- Die Strukturspannungsvariante für Feinblechverbindungen des Fahrzeugbaus (Bauteildicken < $3 \mathrm{~mm}$ ), die von Spannungen in Schalenelementen ausgeht [Fer01]

- Die Hot-Spot-Variante für Rohrknoten aus Grobblechverbindungen, bei der aus einer meistens linearen Extrapolation des Spannungsverlaufes außerhalb der Schweißnaht nach einer vorgegebenen Richtlinie eine (fiktive) Hot-Spot-Spannung $\sigma_{\mathrm{hs}}$ ermittelt wird [Rad98-2, Hob05].

- Das Strukturdehnungskonzept nach Haibach, das die in einem definierten, von der Blechdicke abhängigen Abstand zur Nahtübergangskerbe eine reelle Strukturdehnung festlegt. Aus dieser Dehnung lässt sich die Strukturspannung $\sigma_{s}$ Bild 2.4, berechnen. Dieses Konzept lässt sich sowohl für Grob- als auch für Feinbleche anwenden [Hai89].

Das Strukturspannungskonzept ist für die industrielle Nutzung von besonderer Bedeutung, da es im Vergleich zum Kerbspannungskonzept einen deutlich geringeren Modellierungsaufwand erfordert. So lassen sich die maßgebenden Strukturspannungen beispielsweise durch FiniteElement-Berechnungen mit einem verhältnismäßig grob vernetzten Schalenmodell ermitteln. 
Vorrangig wird in der industriellen Praxis auf die Hot-Spot-Variante zurückgegriffen, die bereits Bestandteil moderner Regelwerke ist [Eur92, Eur98, Hob03, FKM02].

\subsubsection{Strukturspannungskonzept nach IIW-Empfehlung - Grobbleche}

Generell wird zwischen Schweißverbindungen an Rohrknoten (Siehe Abschnitt 2.2.5) und in anderen Konstruktionen unterschieden. Die folgenden Ausführungen beziehen sich auf letztere, wobei plattenartige Strukturen im Vordergrund der Anwendungen stehen.

Die 'klassische' Methode der Strukturspannungsermittlung besteht in der linearen oder quadratischen Extrapolation von Messwerten über zwei oder drei Punkte zum Nahtübergang, Bild 2.4, für deren Lage in [Hob05] Empfehlungen gegeben werden. Die Oberflächenextrapolation der Spannungen lässt sich entsprechend auf Finite-Elemente-Analysen anwenden, Bild 2.5a. Alternativ bietet sich bei Schalen- bzw. Plattenstrukturen die sog. Innenlinearisierung an, die bei einer Volumenmodellierung die Anordnung von drei oder mehr Elementen in Dickenrichtung erfordert, Bild 2.5b. Systematische Reihenuntersuchungen haben gezeigt, dass detaillierte Regeln zur FiniteElemente-Modellierung und zur Spannungsauswertung erforderlich sind, um große Streuungen und Unsicherheiten in den Ergebnissen zu vermeiden [Nie95, Fri02, Nie04].

a. Außenlinearisierung an der Oberfläche

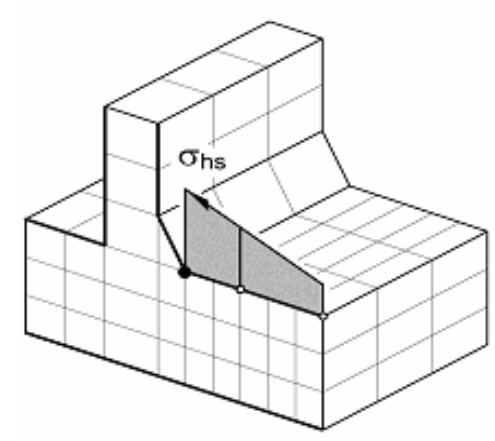

b. Innenlinearisierung

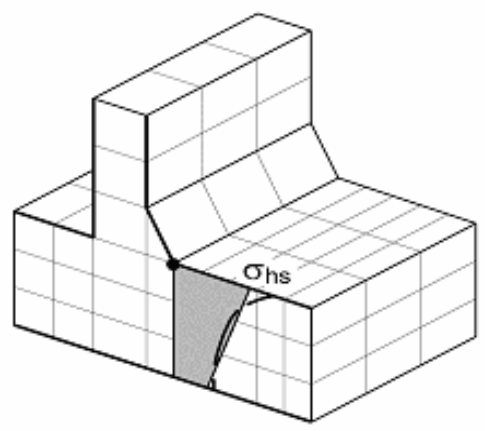

c. Innenlinearisierung im Abstand $\delta$

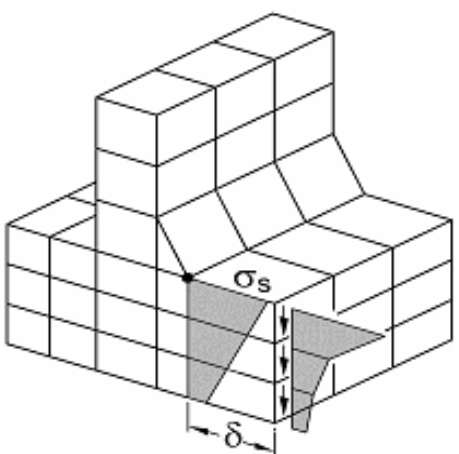

DIA 7639d

Bild 2.5: Lineare Extrapolation von Oberflächenspannungen und Innenlinearisierung in einem FE-Modell

Die anzusetzenden Entwurfswöhlerlinien wurden auf der Basis einer umfangreichen Auswertung von Schwingfestigkeitsversuchen [Mad02] festgelegt. Nach [Nie04] wird im Normalfall für Schweißverbindungen an Stahl die Schwingfestigkeitsklasse FAT $100 \quad(=$ Schwingfestigkeitskennwert in MPa bei $2 \cdot 10^{6}$ Schwingspielen) empfohlen. Ausnahmen bilden kraftübertragende Kehlnähte (wegen der höheren lokalen Spannungskonzentration am Nahtübergang) sowie längere Steifen an Plattenkanten (mit I $>100$ mm), für die FAT 90 gilt, Bild 2.6 [Mad02]. Für Aluminium könnten dementsprechend die Klassen FAT 40 und FAT 36 angenommen werden. 


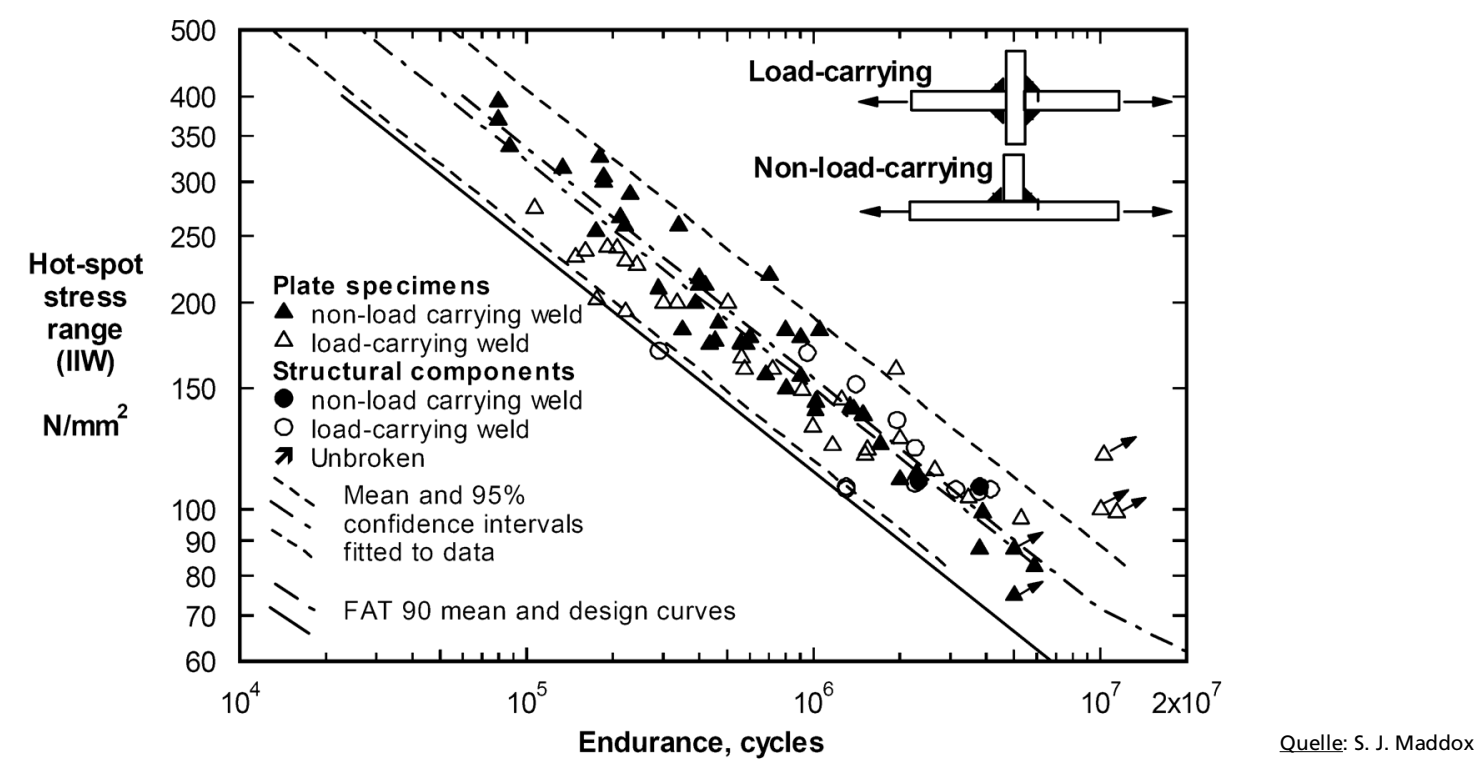

DIA 7642d

Bild 2.6: Ertragbare Strukturspannungen für kraft- und nicht kraftübertragende Kehlnähte

Bei Schweißverbindungen an Plattendicken von über $25 \mathrm{~mm}$ ist der die Schwingfestigkeit mindernde Dickeneinfluss zu berücksichtigen:

$\Delta \sigma(\mathrm{t}>25 \mathrm{~mm})=\Delta \sigma\left(\mathrm{t}_{0}=25 \mathrm{~mm}\right)\left(\mathrm{t}_{0} / \mathrm{t}\right)^{\mathrm{n}}$.

Der Exponent variiert zwischen $\mathrm{n}=0,1$ für Nahtübergänge an Plattenkanten, $\mathrm{n}=0,2$ für Stumpfstöße und $n=0,3$ für sonstige Verbindungen [Nie04].

Ein besonderes Problem stellen fertigungsbedingte Vorverformungen dar, die z. B. in Form von Kantenversatz und Winkelschrumpfung auftreten. Da die Basis der Entwurfswöhlerlinien gemessene Strukturspannungen sind, welche die Auswirkungen eventueller Vorverformungen der Versuchsproben bereits beinhalten, sind im Anwendungsfall deren Auswirkungen in den Strukturspannungen explizit zu berücksichtigen. Beim Nennspannungskonzept sind sie dagegen bis zu einer bestimmten Höhe implizit in den Wöhlerlinien enthalten.

Im Normalfall werden allerdings die Spannungen mit 'perfekten' FE-Modellen ermittelt, welche keine Vorverformungen enthalten. Die Auswirkungen der Vorverformungen müssen in der Strukturspannung vor allem bei Stumpf- und Kreuzstößen (wegen des möglichen Versatzes) sowie bei einseitigen, quer zur Beanspruchung liegenden Kehlnähten (wegen der möglichen Winkelschrumpfung) in plattenartigen Strukturen berücksichtigt werden. Liegen keine detaillierten Werte vor, empfiehlt der Internationale Schweißverband [Hob05], die Axialspannung an den Verbindungen mit gegebenen Faktoren zu multiplizieren, welche die Auswirkung von Kantenversätzen in Höhe von 5-15\% der Plattendicke beinhalten.

\subsubsection{Strukturspannungskonzept nach Dong}

Das Konzept der Innenlinearisierung über der Plattendicke wurde von Dong aufgegriffen und dahin gehend erweitert, dass insbesondere der Einfluss des Spannungsgradienten in Richtung des erwarteten Rissfortschritts auf bruch-mechanischer Basis berücksichtigt wird [Don01, Don02]. Die Innenlinearisierung über die Dicke einer Platte mit einseitiger Schweißnaht ist in Bild 2.7a dargestellt. In bestimmten Fällen wird eine Linearisierung bis zu einer Tiefe $t_{1}<t$ empfohlen, Bild $2.7 \mathrm{~b}$, beispielsweise bei Anrissen von der Plattenkante, wobei $t_{1}$ die Endrisslänge darstellt. Bei 
beidseitigen Schweißverbindungen unter symmetrischer Belastung wird eine Linearisierung über die halbe Plattendicke vorgeschlagen $\left(t_{1}=t / 2\right)$, wodurch sich eine veränderte Strukturspannungsdefinition gegenüber dem herkömmlichen Konzept ergibt, Bild 2.7c. Prinzipiell erfolgt die Linearisierung nach Dong nur über eine monoton fallende Spannungsverteilung.

a. Innenlinearisierung bei einseitiger Naht

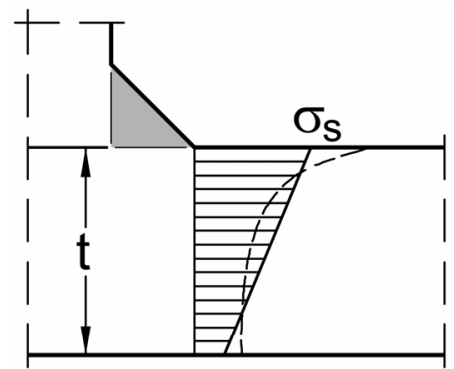

b. Innenlinearisierung bei definierter Tiefe

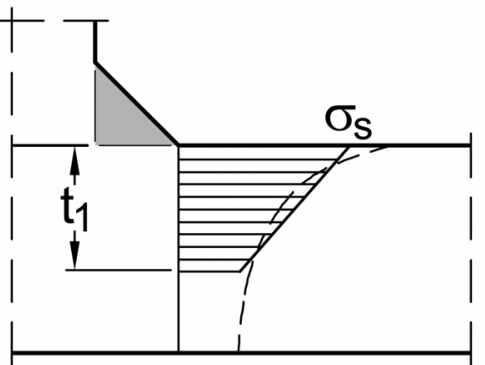

c. Innenlinearisierung bei beidseitiger Naht

DIA 7643d

\section{Bild 2.7: Definition der Strukturspannung nach Dong für verschiedene Fälle}

Für die Ermittlung der Strukturspannung aus FE-Netzen schlägt Dong spezielle Prozeduren vor, die als relativ netzunempfindlich angesehen werden. Da Elementspannungen stark von der Netzfeinheit abhängen und in der Nähe des Nahtübergangs von der Spannungssingularität beeinflusst werden, sollen diese in einem Abstand $\delta$ vom Nahtübergang entfernt ausgewertet werden, Bild 2.7c. Über Gleichgewichtsbedingungen können dann aus den Normal- und Schubspannungen der Membran- und Biegeanteil und damit die lineare Spannungsverteilung in dem Schnitt am Nahtübergang ermittelt werden. Vernachlässigt werden dabei die Spannungen an den anderen Elementseiten, was Ungenauigkeiten bei hoher Spannungskonzentration verursacht [Fri04]. Bei einer Teillinearisierung bis zur Tiefe $t_{1}$ müssen zusätzlich die Spannungsanteile an der Unterseite des Bereiches $\delta \times \mathrm{t}_{1}$ in die Gleichgewichtsbedingungen einbezogen werden. Alternativ schlägt Dong vor, die Strukturspannungen aus den inneren Knotenkräften in dem Schnitt am Nahtübergang zu ermitteln, da diese generell das Gleichgewicht erfüllen. Dieses Vorgehen empfiehlt sich besonders bei Schalenmodellen, bei denen natürlich eine Teillinearisierung in Dickenrichtung ausgeschlossen ist.

Zur Ermittlung der ertragbaren Schwingspielzahlen wird eine "Master-Wöhlerlinie" mit einem speziellen Strukturspannungsparameter $\Delta \mathrm{S}_{\mathrm{s}}$ verwendet, der sich aus der Strukturspannungsschwingbreite $\Delta \sigma_{s}$ wie folgt ergibt:

$\Delta S_{s}=\Delta \sigma_{s} \cdot\left(\frac{t}{t_{0}}\right)^{\frac{m-2}{m}} \cdot \mid(r)^{-\frac{1}{m}}$

mit der Plattendicke $t$, der Bezugsdicke $t_{0}=1 \mathrm{~mm}$ (in Anlehnung an [Don02] erweitert), dem Exponent $m$ der Paris-Gleichung zur Beschreibung des Rissfortschritts (mit $m=3,6$ nach Dong) und einem Integralwert I $(r)$, der vom Verhältnis $r=\sigma_{b} /\left(\sigma_{m}+\sigma_{b}\right)$ der Biegespannung zur gesamten Strukturspannung sowie von Randbedingungen während des Rissfortschritts abhängt (last- oder verformungsgesteuert). Die "Master-Wöhlerlinie", Bild 2.8, ist durch Auswertung von einer 
Vielzahl von Versuchen ermittelt worden, wobei jeweils die Strukturspannung entsprechend Bild 2.7 sowie der Strukturspannungsparameter nach Gl. 2.1 ermittelt wurde. Die Streuung der Spannung beträgt $T_{s s}=1: 2,6$ und ist für Schweißverbindungen als groß anzusehen. Darüber hinaus wurden Vorverformungen nicht explizit behandelt, $d$. h. sie beeinflussen die "MasterWöhlerlinie" in dem Maße, wie sie bei den Versuchen vorgelegen haben.

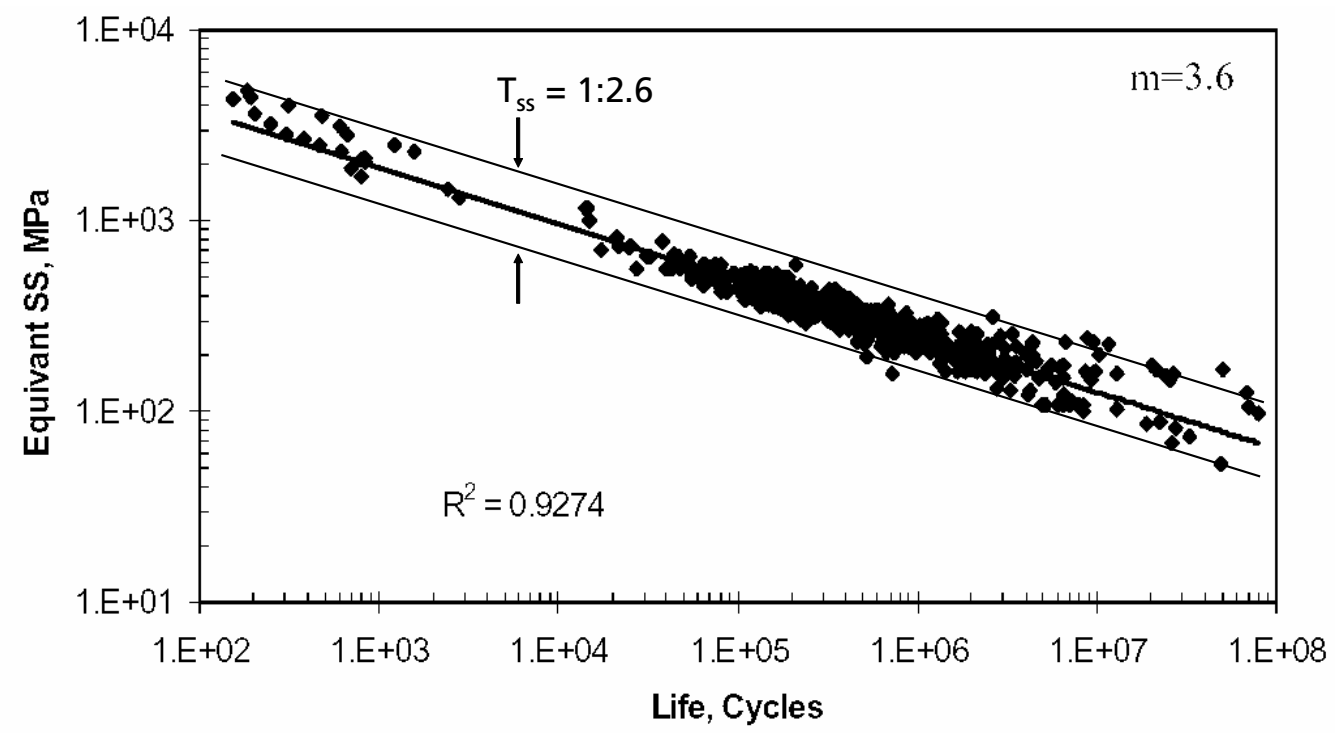

Quelle: P. Dong et al

DIA 7644d

Bild 2.8: Master-Wöhlerlinie nach Dong als Funktion des Struktursp. Parameters $\Delta \mathbf{S}_{\mathrm{s}}$

Bei der Bewertung dieses Konzepts ist zurzeit noch Vorsicht geboten. Die Bilinearisierung (oder Teillinearisierung) der Strukturspannungen setzt die Festlegung einer ermüdungswirksamen Basislänge voraus, über der linearisiert wird. Eine solche lässt sich jedoch nicht einheitlich festlegen, sondern nur fallweise in Anpassung an vorliegende Versuchsergebnisse.

\subsubsection{Strukturspannungskonzept nach Xiao und Yamada}

Vor dem Hintergrund der immer leistungsfähigeren Soft- und Hardware ist ein neuartiges Strukturspannungskonzept vorgeschlagen worden [Xia04], welches den berechneten Spannungswert an einem Punkt, der sich in einer Tiefe von $1 \mathrm{~mm}$ unterhalb des Nahtübergangs in Richtung des erwarteten Rissfortschritts befindet, als relevanten Parameter für die Schwingfestigkeit annimmt, vorerst beschränkt auf nicht-tragende Kehlnähte an beidseitigen Quer- und Längssteifen. 


\section{a. Entlang der Oberfläche}

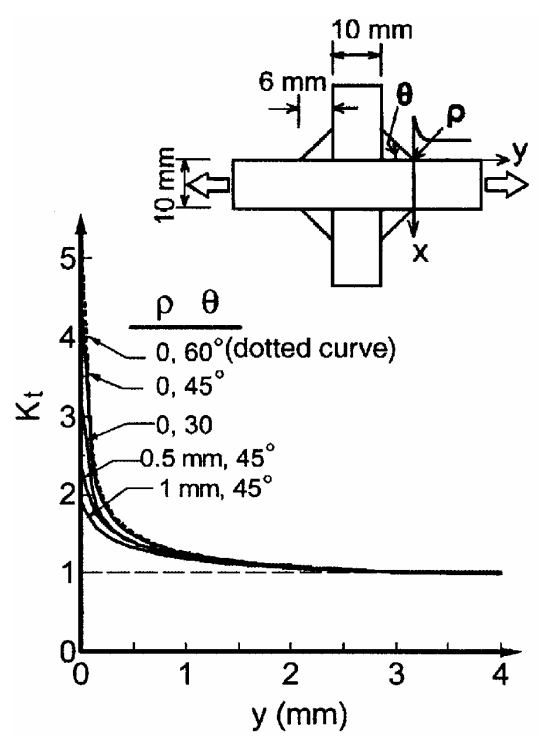

b. In Dickenrichtung

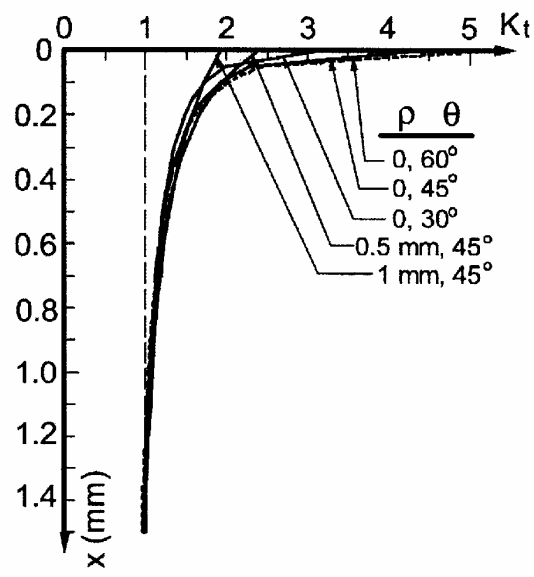

Quelle: Z.-G. Xiao, K. Yamada

DIA 7645d

Bild 2.9: Spannungsverteilung nach Xiao und Yamada

Die Wahl dieses Punktes wird anhand eines Referenzdetails begründet, einer $10 \mathrm{~mm}$ dicken Platte mit beidseitigen Quersteifen, Bild 2.9. FE-Berechnungen haben gezeigt, dass die lokale Spannung am Nahtübergang des Referenzdetails viel schneller in Tiefenrichtung abfällt als an der Oberfläche. Während an der Oberfläche die lokale Spannungserhöhung in einer Entfernung von ca. 2,5 mm abgeklungen ist, wird in Tiefenrichtung die Nennspannung schon bei ca. $1 \mathrm{~mm}$ erreicht, und zwar unabhängig von der örtlichen Ausbildung des Nahtübergangs, Bild 2.9b. Außerdem wird gezeigt, dass die 1-mm-Spannung insbesondere die frühe Rissfortschrittsphase gut repräsentiert. Bei FE-Analysen wird eine Netzteilung benötigt, welche die 1-mm-Spannung mit genügender Genauigkeit bestimmt. Als maximale Elementkantenlänge wird $1 \mathrm{~mm}$ genannt.

Das Konzept wurde auf verschiedene Verbindungstypen übertragen, deren Geometrien zum Referenzdetail ähnlich sind, d. h. Längs- und Quersteifen auf durchlaufenden Platten aufweisen. Die in Versuchen ermittelten Lebensdauern, aufgetragen in Abhängigkeit der jeweils berechneten 1-mm-Spannung, bilden ein relativ schmales Streuband, dessen Untergrenze der Entwurfswöhlerlinie entsprechend FAT 100 entspricht, Bild 2.10. Außerdem wird gezeigt, dass mit der 1-mm-Spannung der Dickeneinfluss besser berücksichtigt wird als mit dem herkömmlichen oder mit Dong's Strukturspannungskonzept. 


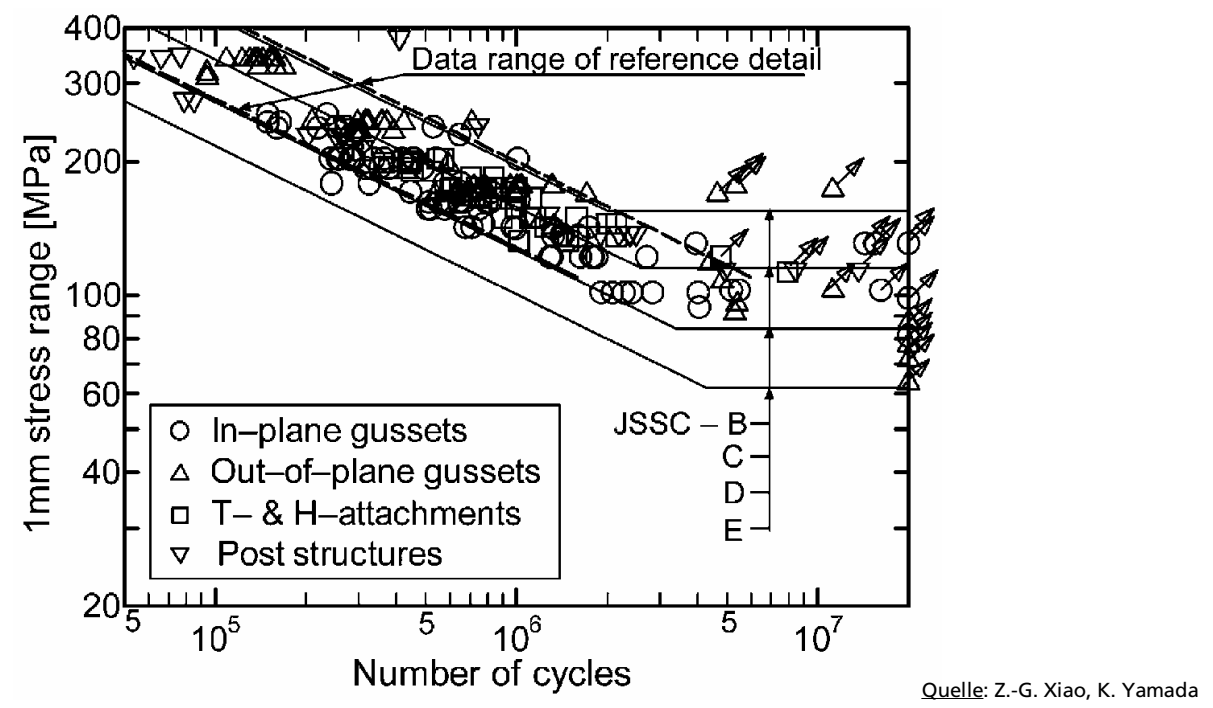

DIA 7646d

Bild 2.10: Ertragbare Lebensdauern verschiedener Strukturdetails in Abhängigkeit von der $1 \mathrm{~mm}$ unterhalb des Nahtübergangs ermittelten Längsspannung (Werkstoff: Stahl)

Die Überprüfung der Anwendbarkeit des Konzeptes auf andere Verbindungstypen (z. B. Kreuzstöße mit kraftübertragenden Kehlnähten oder einseitige Längs- und Quersteifen) und Lastfälle (z. B. mit überwiegend Biegung) steht noch aus.

\subsubsection{Strukturspannungskonzept nach Fermer und Svensson - Feinbleche}

Dieses dem Automobilbau zuzuordnende Verfahren für Feinblechverbindungen [Fer01] mit $\mathrm{t} \leq 3 \mathrm{~mm}$ geht von einem Schalenmodell aus, Bild 2.11. Die Struktur wird mit Schalenelementen vernetzt, wobei die Schweißnahtelemente die doppelte Dicke des dünneren Blechs haben sollen. In Nahtrichtung soll die Elementlänge ca. $5 \mathrm{~mm}$ betragen. Der Mittelwert der Spannungen in den an der Schweißnaht angrenzenden Knoten wird als Strukturspannung bewertet. Aus Versuchen an verschiedenen Proben mit unterschiedlichen Blechdicken liegen für Aluminium und Stahl jeweils zwei Wöhlerlinien zu Bemessung vor. Die sogenannte "flexible" Wöhlerlinie gilt, wenn überwiegend Plattenbiegung vorliegt, während die "steife" Wöhlerlinie für Axialbelastung (Membranspannungszustände) herangezogen wird. Eine Anwendung wurde bereits in [Bür01] gezeigt.

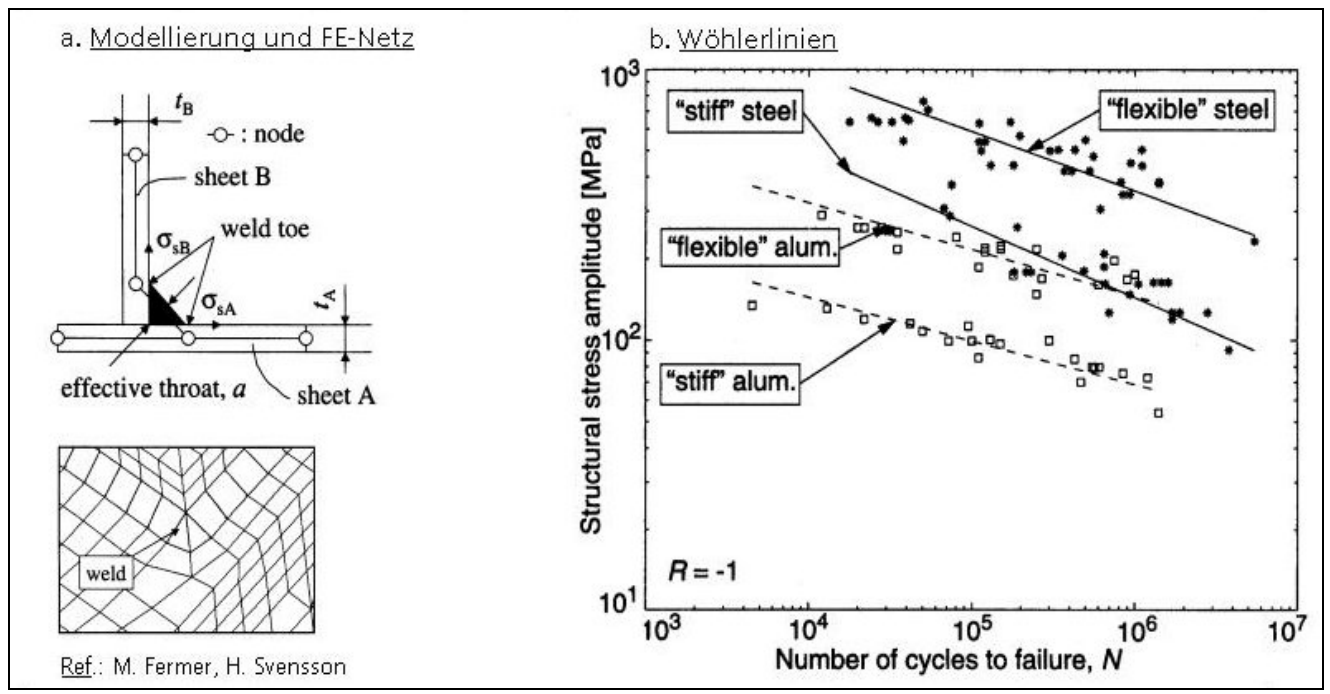

Bild 2.11: Strukturspannungskonzept für geschweißte Feinbleche 


\subsubsection{Hot-Spot-Strukturspannungskonzept nach Dijkstra und de Back}

Das Hot-Spot-Strukturspannungskonzept nach Dijkstra und de Back bezieht sich auf geschweißte Rohrknoten der Offshoretechnik, Bild 2.4. Der Begriff "Hot Spot" steht mit der lokalen Temperaturerhöhung infolge plastischer Formänderung bei der Risseinleitung in Zusammenhang. Die Ermüdungsfestigkeit von geschweißten Rohrknoten lässt sich nur auf Basis der Strukturspannungen realistisch bewerten, weil neben den Kerbspannungen durch die lokale Biegung der Rohrschalen unterschiedliche Spannungserhöhungen entstehen, welche in den Nennspannungen unberücksichtigt bleiben. Das Konzept basiert auf der Bestimmung der Strukturspannung am Risseinleitungsort, welche mit der ertragbaren Nennspannung einer geometrisch ähnlichen Schweißprobe verglichen wird, um die Ermüdungsfestigkeit des Rohrknotens zu bewerten [Rad98-2].

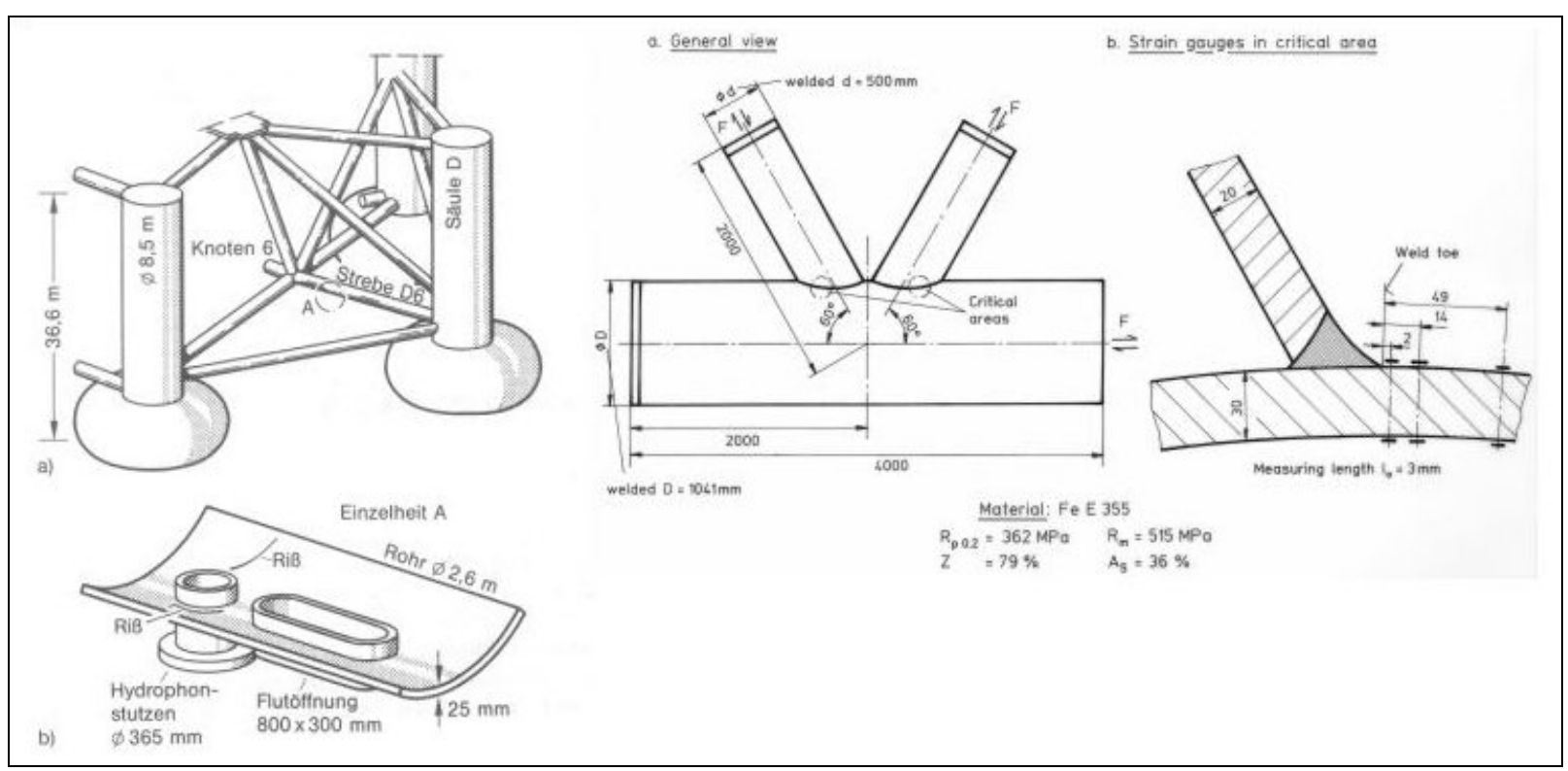

Bild 2.12: Hot-Spot-Konzept bei Rohrknoten an Off-Shore-Konstruktionen 


\subsubsection{Strukturdehnungskonzept nach Haibach}

Nach Haibach [Hai89, Hai68, Rad98-2] kennzeichnet die mit einem Dehnungsmessstreifen in geringem Abstand vom Nahtübergang gemessene Dehnung die Langzeitermüdungsfestigkeit von Schweißverbindungen unabhängig von der Nahtart und der Belastungsart, vorausgesetzt der Riss wird am Nahtübergang eingeleitet, Bild 2.13.

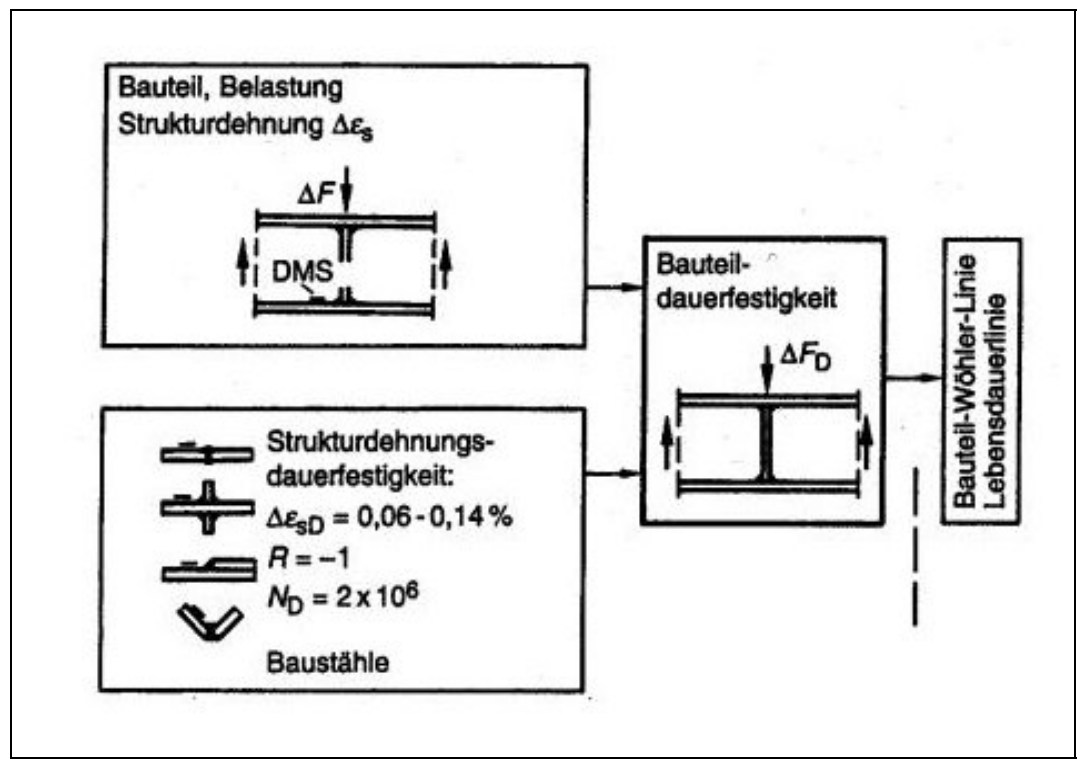

Bild 2.13: Strukturdehnungskonzept nach Haibach

Der Abstand des Dehnungsmessstreifens von der Nahtübergangskerbe ist dabei so festgelegt, dass die gemessene Strukturspannung als "lokale Nennspannung" zu betrachten ist, deren ertragbarer Wert aus Wöhlerversuchen mit herkömmlichen Schweißnahtproben abgeleitet wird. Die Empfehlung von Haibach, Dehnungsmessstreifen (DMS) mit einer Länge von $3 \mathrm{~mm}$ in einer Entfernung von 1,5 bis 2,0 mm (Mitte des DMS) von der Nahtübergangskerbe zu verwenden, gilt für etwa $10 \mathrm{~mm}$ dicke Platten; für Plattendicken kleiner als $5 \mathrm{~mm}$ werden DMS mit einer Länge von 1,5 mm bei einem Abstand von 0,75 bis 1,0 mm empfohlen, Bild 2.4.

\subsection{Kerbbeanspruchungskonzepte}

\subsubsection{Kerbdehnungskonzept}

Das Kerbdehnungskonzept (auch Kerbgrund- und örtliches Konzept genannt) zur Abschätzung von Schwingfestigkeit und Lebensdauer bis zu einem technischen Anriss geht von den an der höchstbeanspruchten Stelle des Bauteils, Bild 2.4, auftretenden elastisch-plastischen Dehnungsamplituden aus und stellt sie der dehnungsgeregelt aufgenommenen Wöhlerlinie des Werkstoffs gegenüber. Der Grundgedanke des Verfahrens ist, dass sich der Werkstoff hinsichtlich seines Ermüdungsverhaltens am höchstbeanspruchten Element im Kerbgrund genauso verhält wie eine dort gedachte oder tatsächlich entnommene ungekerbte Probe im dehnungsgeregelten Versuch, Bild 2.14. 


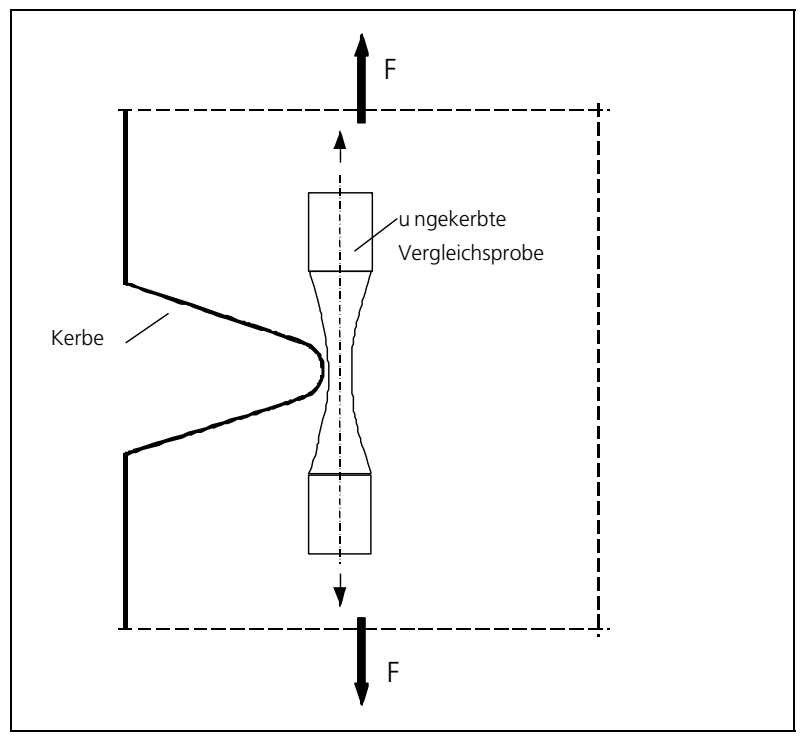

Bild 2.14: Ungekerbte Probe zur Simulation des Verformungsverhaltens

Der Vorteil gegenüber dem Nennspannungskonzept ist, dass die für das Versagen verantwortliche Beanspruchung verwendet wird und die für die Berechnung der Lebensdauer benötigten experimentellen Schwingfestigkeitskennwerte unabhängig vom konkreten Bauteil sind. Einflüsse, welche die Lebensdauer des Bauteils bestimmen wie z.B. Eigenspannungen, können mit dem örtlichen Konzept beschrieben werden.

Im Gegensatz zum Strukturspannungskonzept ist das örtliche Konzept auch dann anwendbar, wenn die versagenskritische Stelle verdeckt liegt und durch die Extrapolation der Strukturspannung nicht erreicht wird, Bild 2.15, [Nie92].

a. Geeignete Fälle für das Strukturspannungskonzept

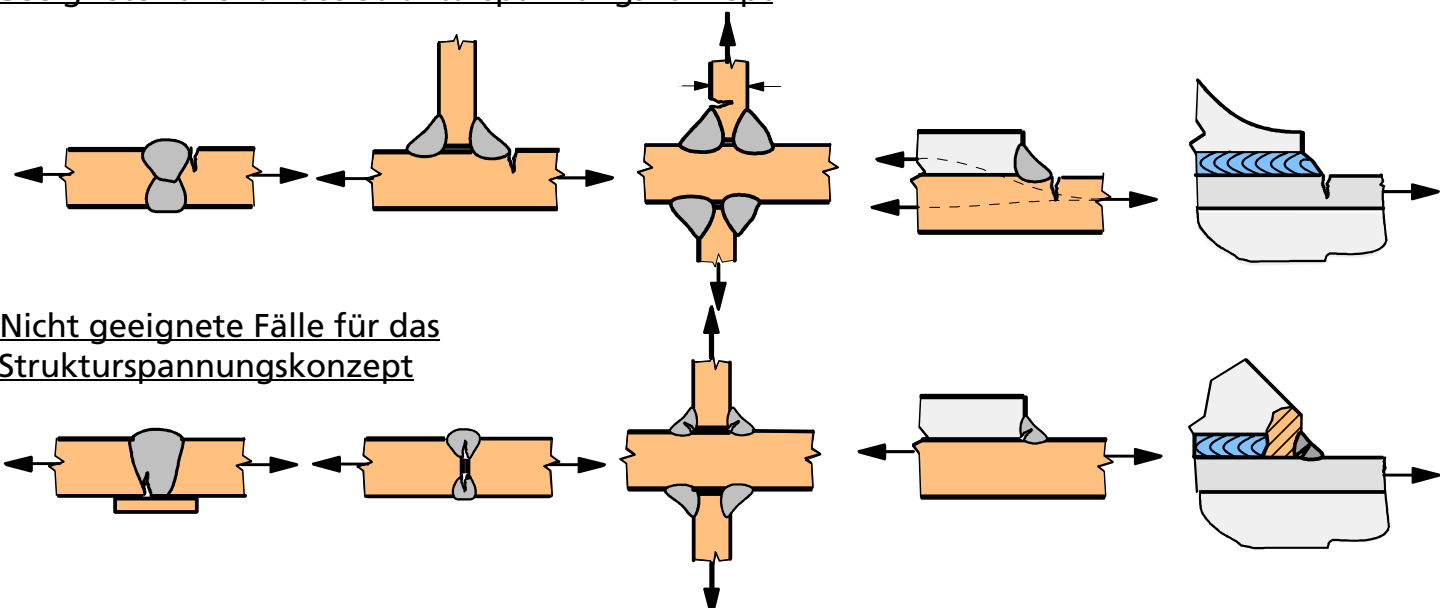

这

Quelle: E. Niemi

Bild 2.15: Anwendungsgrenzen des Strukturspannungskonzeptes

Als Eingangsdaten werden die zyklische Spannungs-Dehnungs-Kurve des Werkstoffs und die dehnungsgesteuert aufgenommene Anrisswöhlerlinie der ungekerbten Probe benötigt. Da die Berechnung nur bis zum Versagen durch Anriss erfolgt, ist eine Rissfortschrittsberechnung anzuschließen, um das Versagen durch Bruch zu erfassen, Bild 2.16, [Son93, Rad95, See96, Soc77, Dow82, Dow87, Bro74, See84]. 


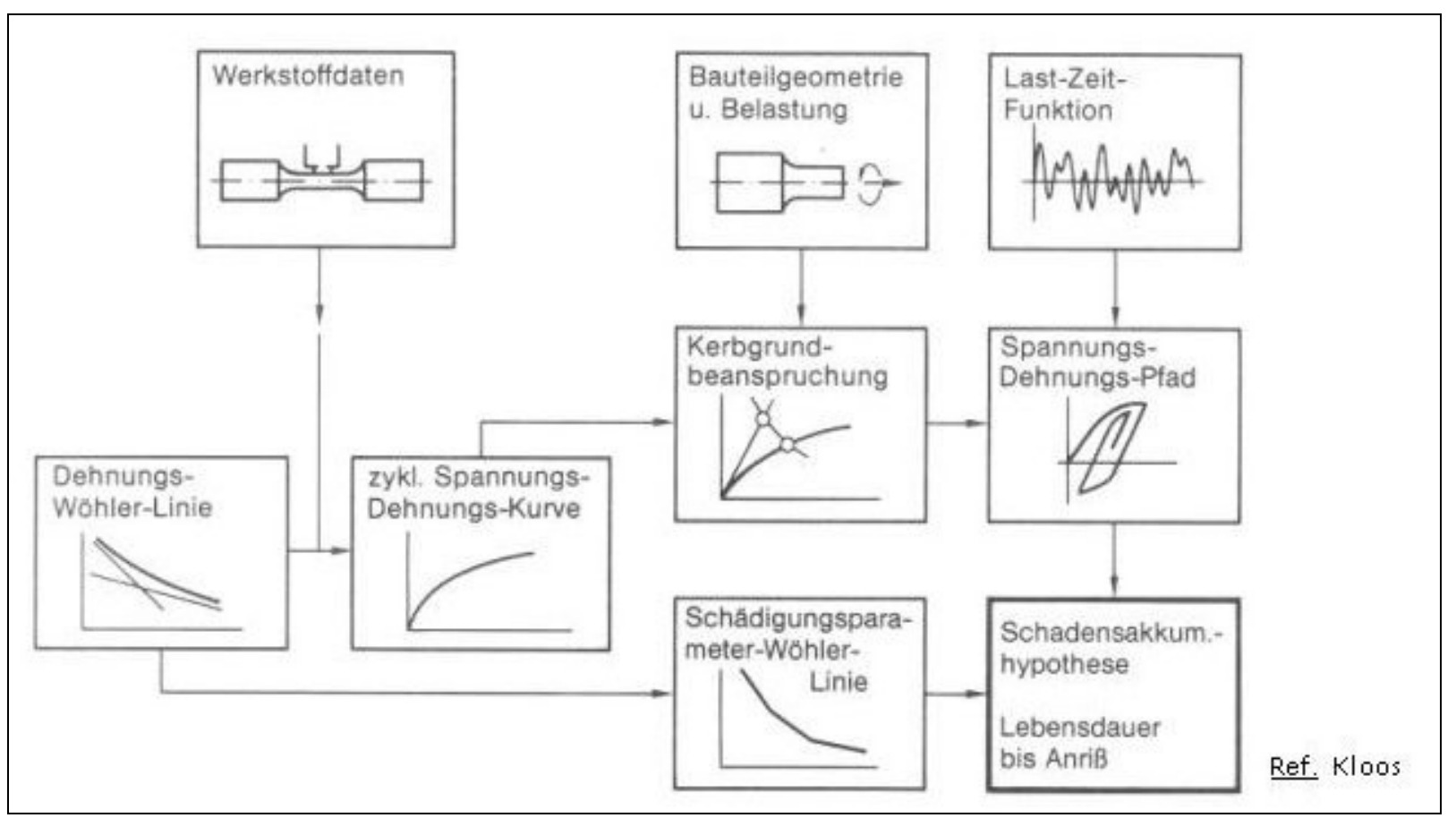

Bild 2.16: Ablaufschema Kerbdehnungskonzept

\subsubsection{Beschreibung des elastisch-plastischen Materialverhaltens}

Das Spannungs-Dehnungsverhalten, welches an ungekerbten Proben im dehnungsgeregelten Schwingfestigkeitsversuch ermittelt wird, Abschnitt 4.1.1, wird durch die Verfestigungskoeffizienten $K, K^{\prime}$ und die Verfestigungsexponenten $n, n^{\prime}$ entsprechend der Ramberg- Osgood- Beziehung berechnet [Ram43]:

$\varepsilon=\frac{\sigma}{\mathrm{E}}+\left(\frac{\sigma}{\mathrm{K}}\right)^{\frac{1}{n}}$

$\varepsilon_{a}=\frac{\sigma_{a}}{E}+\left(\frac{\sigma_{a}}{K^{\prime}}\right)^{\frac{1}{n^{\prime}}}$

Nach Coffin, Manson und Morrow lassen sich die Werte der elastischen und der plastischen Dehnungsamplitude der Anrisswöhlerlinie im doppeltlogarithmischen Maßstab durch eine Gerade beschreiben [Cof54, Man65, Mor65]:

$$
\varepsilon_{a, e l}=\frac{\sigma_{f}^{\prime}}{E} \cdot\left(2 \cdot N_{A}\right)^{b} \quad \text { für den elastischen Anteil mit }
$$

$$
\sigma_{f}^{\prime} \quad \text { zyklischer Spannungskoeffizient und }
$$

b Zyklischer Spannungsexponent sowie

$$
\varepsilon_{\mathrm{a}, \mathrm{pl}}=\varepsilon_{\mathrm{f}}^{\prime} \cdot\left(2 \mathrm{~N}_{\mathrm{A}}\right)^{\mathrm{c}} \quad \text { für den plastischen Anteil mit }
$$

$\varepsilon_{f}^{\prime} \quad$ zyklischer Dehnungskoeffizient und

c zyklischer Dehnungsexponent. 
Die Gesamtdehnungsamplitude ergibt sich zu:

$$
\varepsilon_{\mathrm{a}, \mathrm{ges}}=\frac{\sigma_{f}^{\prime}}{E} \cdot\left(2 \cdot \mathrm{N}_{\mathrm{A}}\right)^{\mathrm{b}}+\varepsilon_{f}^{\prime} \cdot\left(2 \cdot \mathrm{N}_{\mathrm{A}}\right)^{\mathrm{c}} \text {. }
$$

\subsubsection{Berechnung der örtlichen Beanspruchung und der Anrißlebensdauer}

Die Berechnung der Anrißlebensdauer $\mathrm{N}_{\mathrm{A}}$ basiert auf der Kenntnis des dehnungskontrollierten Schwingfestigkeitsverhaltens der ungekerbten Probe (Gl. 2.7). Für die Bestimmung der örtlichen Beanspruchung an der Versagensstelle wird die Neuber-Regel

$$
\mathrm{K}_{\mathrm{t}} \cdot \sqrt{\Delta \sigma_{\mathrm{n}} \cdot \Delta \varepsilon_{\mathrm{n}}}=\sqrt{\Delta \sigma \cdot \Delta \varepsilon}
$$

angewandt, wobei $\Delta \sigma_{n}$ und $\Delta \varepsilon_{n}$ die Nennspannungs- bzw. Nenndehnungsschwingbreiten und $\Delta \sigma$ und $\Delta \varepsilon$ die örtliche Beanspruchung darstellen. Unter Beachtung des "fatigue notch size effect" leitet Peterson aus der theoretischen Formzahl $K_{t}$ die Kerbwirkungszahl $K_{f}$ ab [Pet59]:

$$
K_{f}=1+\frac{K_{t}(\rho)-1}{1+\frac{\rho^{*}}{\rho}}
$$

$\rho$ bezeichnet den Kerbradius, $\rho^{*}$ entspricht der von Neuber/Radaj eingeführten Ersatzstrukturlänge, die in Abschnitt 2.3.2.1 erläutert und in Abschnitt..2.3.3 ermittelt wird ${ }^{1}$.

Ausgehend von der Beobachtung, dass der Nahtübergangsradius über der Schweißnahtbreite schwankt und sich die Anrisse an kleinen Defekten bilden, ist eine kritische Abschätzung der örtlichen Beanspruchung erforderlich. An den so genannten Fehlstellen (Schweißporen, Mikrolunker) liegt eine wesentlich höhere örtliche Spannung, als sie durch den Kerbradius gegeben ist, vor. Daher wird von Lawrence [Law97, Che79] der Kerbradius $\rho$ in Gl. 2.9 durch die Konstante $\rho^{*}$ ersetzt und der maximale Kerbfaktor $K_{f, \text { max }}$ eingeführt:

$$
K_{f, \max }=1+\frac{K_{t}\left(\rho^{\star}\right)-1}{2}
$$

Die örtliche Beanspruchung lässt sich somit, wie folgt, ableiten

$$
\mathrm{K}_{\mathrm{f}, \max } \cdot \sqrt{\Delta \sigma_{\mathrm{n}} \cdot \Delta \varepsilon_{\mathrm{n}}}=\sqrt{\Delta \sigma \cdot \Delta \varepsilon}
$$

Der Werkstoff verhält sich während des ersten Schwingspiels entsprechend der zügigen $\sigma-\varepsilon-$ Kurve

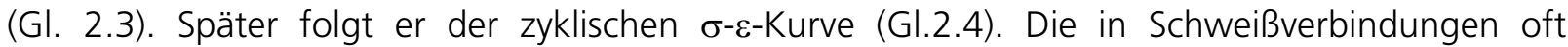
vorhandenen Eigenspannungen können für die Berechnung der örtlichen Beanspruchung in die Erstbelastungskurve einbezogen werden.

In Anwesenheit einer Mittelspannung $\sigma_{m}$ wird der zyklische Spannungskoeffizient $\sigma_{f}^{\prime}$ in (GI. 2.7) um den Betrag von $\sigma_{\mathrm{m}}$ reduziert:

\footnotetext{
1 Beim Mikrostützwirkungskonzept werden die Radien mit $\rho$ bzw. $\rho^{\star}$ bezeichnet; hingegen erfolgt beim Konzept des fiktiven Ersatzradius die Bezeichnung der Radien mit $r$ bzw. $r_{f}$.
} 


$$
\varepsilon_{a, g e s}=\frac{\sigma_{f}^{\prime}-\sigma_{m}}{E} \cdot\left(2 \cdot N_{A}\right)^{b}+\varepsilon_{f}^{\prime} \cdot\left(2 \cdot N_{A}\right)^{c} .
$$

\subsubsection{Kerbspannungskonzept nach Radaj}

Das Kerbspannungskonzept nach Radaj [Rad95-2] beschränkt sich zunächst auf die Ermittlung der sogenannten Dauerfestigkeit von Schweißverbindungen und bietet aber die Möglichkeit für die Erweiterung auf den Zeitfestigkeitsbereich [Wer01]. Es basiert auf der Abschätzung der Mikrostützwirkung an scharfen Übergangskerben des Schweißstoßes nach dem Ansatz von Neuber. Die scharfen Kerben sind fiktiv zu runden, um die ermüdungswirksame einachsige Kerbspannung zu erhalten, welche durch Bezug auf die Nennspannung die Kerbwirkungszahl $\mathrm{K}_{\mathrm{f}}$ des Schweißstoßes ergibt.

$$
K_{f}=\frac{\sigma_{a, n}\left(K_{t}=1 ; \text { Grundwerkstoff o. Schweißgut }\right)}{\sigma_{a}(\text { Schweißprobe })}
$$

Das Konzept ist auf beliebige reale Krümmungsradien anwendbar, wird jedoch häufig als WorstCase-Betrachtung genutzt, indem von dem kleinstmöglichen Krümmungsradius $\rho=0$ ausgegangen wird. Eine fiktive Kerbrundung an Schweißstößen aus Baustahl gibt Radaj mit 1,0 $\mathrm{mm}$ an.

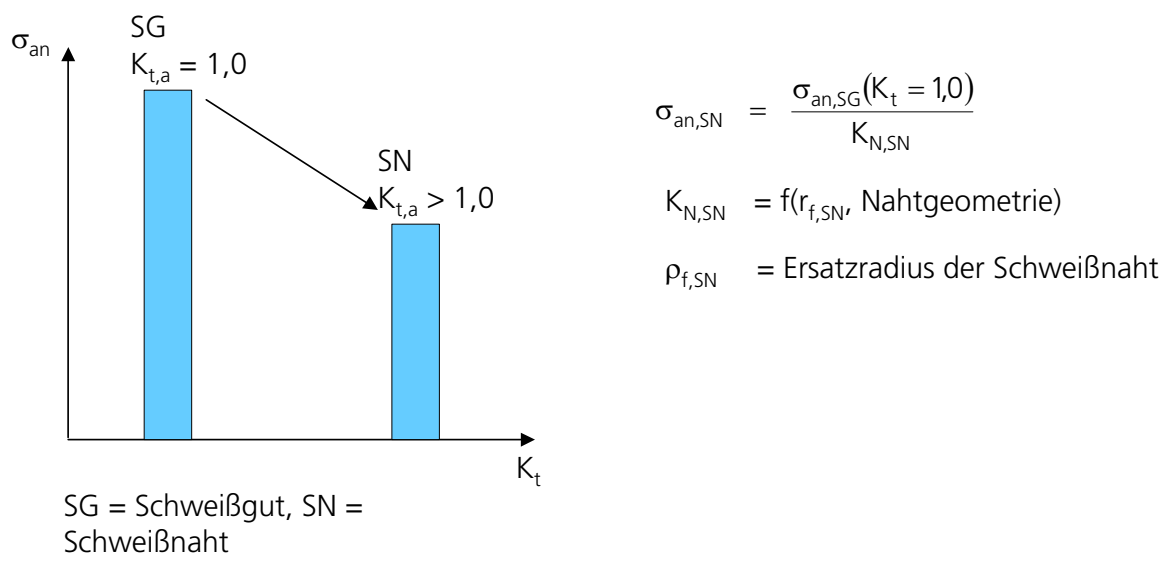

Bild 2.17: Konzept der Mikrostützwirkung an Schweißverbindungen

\subsubsection{Mikrostützwirkung}

Bei Kerben mit sehr kleinen Krümmungsradien treten bei der Berechnung nach der Elastizitätstheorie Spannungssingularitäten auf, d.h. die Spannung nimmt den Wert Unendlich an. Dies liegt darin begründet, dass eine endliche Kraft durch eine sehr kleine Fläche dividiert wird. Die Voraussetzung der Elastizitätslehre, die Strukturlosigkeit des Werkstoffes, ist bei starken Oberflächenkrümmungen nicht zutreffend [Neu68-1]. Um die wahren Spannungsverhältnisse an diesen Stellen besser zu beschreiben, muss auch die Werkstoffstruktur berücksichtigt werden. 
Innerhalb bestimmter kleiner Stoffbereiche bestehen infolge der Struktur unterschiedlich starke Verfestigungen und dadurch größere Verformungswiderstände. Diese Stoffbereiche stützen sich blockweise auf ihre Umgebung ab. Neuber bezeichnet diesen Effekt als Mikrostützwirkung, Bild 2.18, [Neu85, Neu68-1, Neu68-2, Neu61, Neu57] und führt die Ersatzstrukturlänge $\rho^{\star}$ ein.

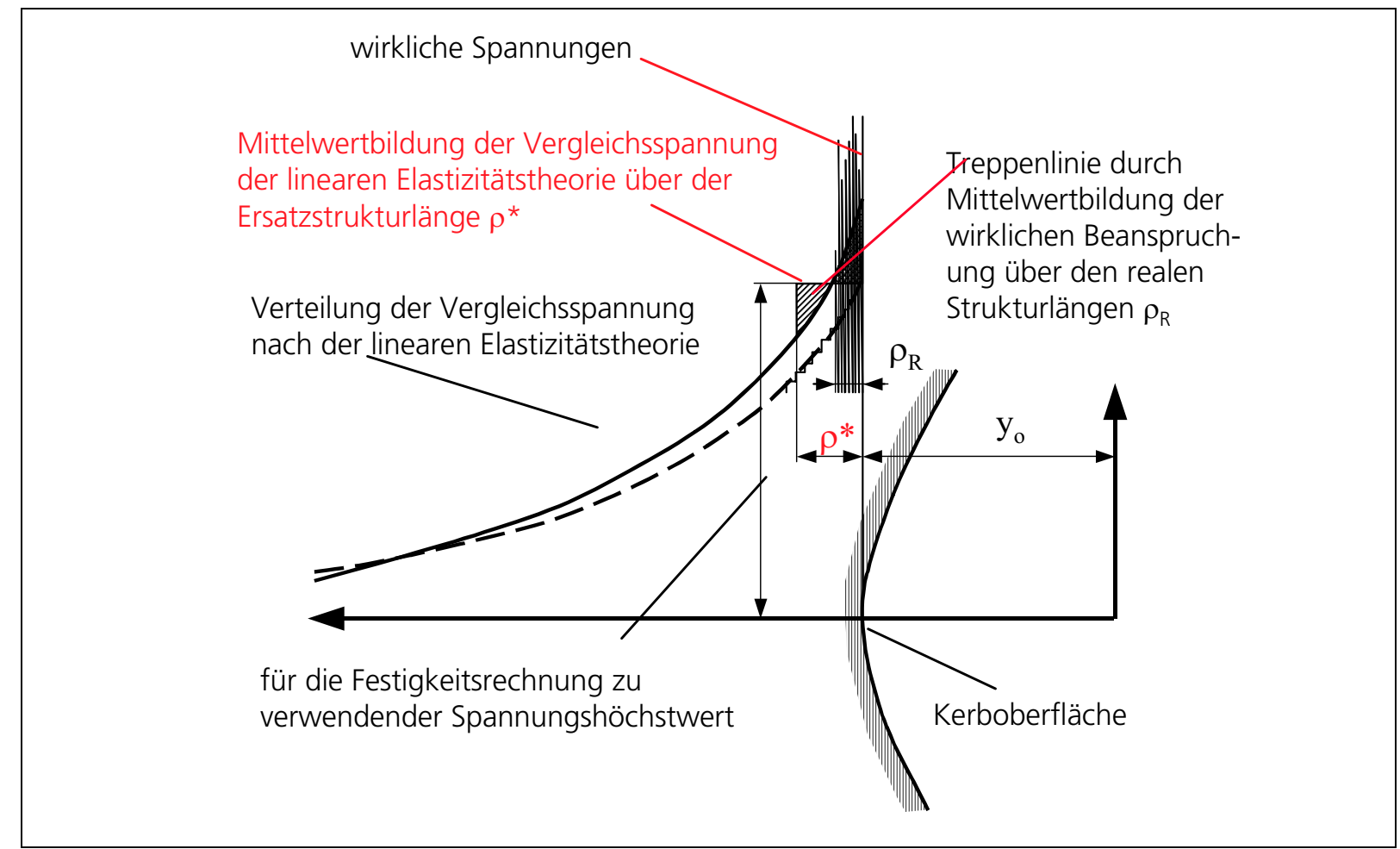

Bild 2.18: Schema der Mittelwertbildung nach Neuber

Die Ersatzstrukturlänge $\rho^{*}$ ist eine in erster Linie von der Struktur und Zusammensetzung des Werkstoffes abhängige Stoffkonstante mit der Dimension einer Länge. Sie dient der Berechnung der maßgeblichen Spannung im Kerbgrund nach der Theorie der Mikrostützwirkung. Dazu wird der Mittelwert der Spannung über der Ersatzstrukturlänge gebildet, wobei nicht die größte Normalspannung herangezogen wird, sondern die Vergleichsspannung, die sich aus der anzuwendenden Festigkeitshypothese ergibt. Somit geht die Mehrachsigkeit der örtlichen Beanspruchung in die Berechnung ein [Neu58].

\subsubsection{Bestimmung der Ersatzstrukturlänge nach Neuber}

Die ursprünglich von Neuber entwickelte Theorie der Mikrostützwirkung sollte nur auf den Bereich der so genannte Dauerfestigkeit angewendet werden und bezog auch keine Schweißverbindungen mit ein.

Die verschiedenen Verfahren zur Bestimmung der Ersatzstrukturlänge $\rho^{*}$ stützen sich auf die Grundlage, dass der Kerbfaktor $K_{N}$, der nach Neuber den Effekt der Mikrostützwirkung berücksichtigt, der Kerbwirkungszahl $K_{f}$ entspricht. Neuber betrachtet die Ersatzstrukturlänge als eine Werkstoff- und Festigkeitsabhängige Größe $\left(\rho^{*}=\rho^{*}\left(R_{\mathrm{p} 0,2}\right.\right.$; Werkstoff $\left.)\right)$. Eine Zuordnung zu Werkstoff und Streckgrenze wurde von Neuber durch experimentelle Untersuchungen durchgeführt, siehe auch Abschnitt 4.1.4 und Bild 4.22. 
$K_{N}=K_{t}\left(\rho_{r}=\rho_{f}\right)=K_{f}=\frac{\sigma_{a, n}\left(K_{t}=1,0\right)}{\sigma_{a, n}\left(K_{t}>1,0\right)}$

Weiter kommt der von Neuber eingeführte fiktive Krümmungsradius $\rho_{\mathrm{f}}$ zur Anwendung, so dass die Ergebnisse und Formeln der klassischen Elastizitätstheorie, insbesondere die Formzahlformeln, ihre Gültigkeit behalten [Neu58]:

$\rho_{f}=\rho_{\mathrm{r}}+s \cdot \rho^{*}$

Der Faktor der Mikrostützwirkung s ist abhängig von der Festigkeitshypothese, von der Art der Belastung und von der Probenform. Für die vorliegende Aluminiumlegierung AlMgSi1 T6 (AW6082 T6) kann die Hypothese der Gestaltänderungsenergie als zutreffende Festigkeitshypothese gewählt werden, da bei zähem Material und konstanten Hauptspannungsrichtungen die Vergleichsspannung nach dieser Hypothese gültig ist [Wel76, Son85, Mag87]. Der Faktor der Mikrostützwirkung s beträgt in diesem Fall 2,5 [Neu68-1, Rad88-1], Tabelle 2.2.

\begin{tabular}{|l|c|c|c|}
\hline & \multicolumn{2}{|c|}{$\begin{array}{c}\text { Zug und Biegung } \\
\text { Rundstäbe mit } \\
\text { Umlaufkerbe }\end{array}$} & $\begin{array}{c}\text { Schub und } \\
\text { Torsion }\end{array}$ \\
\hline Festigkeitshypothese & Flachstäbe & 2 & 1 \\
\hline Schubalspannungshypothese & 2 & $\frac{2-v}{1-v}$ & 1 \\
\hline $\begin{array}{l}\text { Oktaederschubspannungshypothese und Hypothese der } \\
\text { Gestaltänderungsenergie }\end{array}$ & $\frac{5}{2}$ & $\frac{5-2 \cdot v+2 \cdot v^{2}}{2-2 \cdot v+2 \cdot v^{2}}$ & 1 \\
\hline Dehnungshypothese & $2+v$ & $\frac{2-v}{1-v}$ & 1 \\
\hline$v$ Querkontraktionszahl & & & \multicolumn{2}{|c|}{ nach Neuber } \\
\hline
\end{tabular}

Tabelle 2.2: Faktor s der Mikrostützwirkung in Abhängigkeit der Schadenshypothese

Zur Berücksichtigung des Effektes der Mikrostützwirkung führt Neuber [Neu68-1] die Formel

$K_{N}=K_{f}=K_{t} \cdot \sqrt{\frac{\rho_{r}}{\rho_{f}}}$

GI. 2.16

ein. Diese ist aus der Formel für die Formzahl einer scharfen Kerbe durch vereinfachung abgeleitet. Mit Hilfe der Gleichungen Gl. 2.15 und Gl. 2.16 ergibt sich daraus die Ersatzstrukturlänge zu [Wer99]

$\rho^{*}=\frac{\rho_{\mathrm{r}}}{\mathrm{S}} \cdot\left[\left(\frac{\mathrm{K}_{\mathrm{t}}}{\mathrm{K}_{\mathrm{f}}}\right)^{2}-1\right]$. 


\subsubsection{Bestimmung der Ersatzstrukturlänge nach Radaj}

Radaj erweitert das Konzept der Mikrostützwirkung von Neuber auf das Gebiet der Schweißnähte, aber auch nur im Bereich der so genannten Dauerfestigkeit.

Neuber weist darauf hin, dass bei starker Krümmung der Kerbfaktor $K_{N}$ mit Hilfe der Gleichung für die Formzahl berechnet werden kann, in dem anstelle des realen Krümmungsradius der fiktive Krümmungsradius eingesetzt wird [Neu68-1]. Radaj legt die Gleichung für die Formzahl für das quer zur Stabachse liegende Langloch (elliptische Innenkerbe) zugrunde [Rad95, Neu85].

$$
K_{t}=1+2 \cdot \sqrt{\frac{S}{2 \cdot \rho_{r}}}
$$

Es ergibt sich als Gleichung für den Kerbfaktor $\mathrm{K}_{\mathrm{N}}$

$$
K_{N}=1+\left(K_{t}-1\right) \cdot \sqrt{\frac{\rho_{r}}{\rho_{f}}}
$$

und mit Gl. 2.15 und Gl. 2.16 die Ersatzstrukturlänge:

$$
\rho^{\star}=\frac{\rho_{\mathrm{r}}}{\mathrm{S}} \cdot\left[\frac{\left(\mathrm{K}_{\mathrm{t}}-1\right)^{2}}{\left(K_{\mathrm{f}}-1\right)^{2}}-1\right] .
$$

Die Ersatzstrukturlänge wird anhand der Schwingfestigkeitsergebnissen von verschieden gekerbten Proben des Grundwerkstoffes und des Schweißgutes in Abschnitt 4.1.3 berechnet. Da die Formel nach Radaj eine Verallgemeinerung der Formel von Neuber für beliebige $K_{t}-$ und $\mathrm{K}_{\mathrm{f}}$-Werte darstellt, erfolgt die Auswertung in dem genannten Abschnitt nur nach Radaj. Für große Werte geht die Radaj-Formel in die Neuber-Formel über.

Basierend auf der Theorie der Mikrostützwirkung von Neuber [Neu58] ergibt sich der fiktive Kerbgrundradius aus der oben genannten Beziehung, Gl. 2.15. Für den ungünstigen Fall einer Kerbe, nämlich eines Risses wird $\rho_{\mathrm{r}}=0$. Somit vereinfacht sich die genannte Beziehung zu:

$$
\rho_{f}=\rho^{*} \cdot S
$$

Radaj hat für Stahlschweißverbindungen die Ersatzstrukturlänge $\rho^{\star}=0,4 \mathrm{~mm}$ und damit einen fiktiven Ersatzradius von $\rho_{f}=1,0 \mathrm{~mm}$ nach dem Mikrostützwirkungskonzept und mit der WorstCase-Betrachtung $\rho_{\mathrm{r}}=0 \mathrm{~mm}$ abgeleitet. Die Untersuchungen wurden mit Stahlguss St37 mit $R_{p 0,2}$ $=240 \mathrm{MPa}$ durchgeführt.

\subsubsection{Kerbgrundkonzept mit fiktivem Ersatzradius nach Seeger}

Die Ersatzstrukturlänge $\rho *$ ist im Falle einer Schweißverbindung vom jeweiligen Gefüge der Bruchausgangsstelle abhängig, d.h. vom Schweißgut oder von der Wärmeeinflusszone und außerdem von der örtlichen chemischen Zusammensetzung des Werkstoffes. Demzufolge erfordert die Anwendung dieses Konzeptes die genaue Kenntnis des Gefüges in der Bruchausgangsstelle und somit der zugehörigen Ersatzstrukturlänge [Mor04, Mor04-1]. Da die Ermittlung dieses Wertes mit einem großen Aufwand verbunden ist, ist dieses Konzept ingenieurmäßig nicht geeignet. 
Aufgrund von umfangreichen Untersuchungen an Baustählen wurde für Schweißverbindungen aus Stahl mit Blechdicken $t \geq 5 \mathrm{~mm}$ das Kerbgrundkonzept mit dem fiktiven Radius $r_{f}=1,0 \mathrm{~mm}$, welches werkstoffunabhängig ist, abgeleitet [Oli89, See96]. D.h., mit einer auf diesem Radius basierenden Modellierung ließen sich die Untersuchungsergebnisse von verschiedenen Schweißnahtgeometrien in ein enges Wöhlerlinienstreuband überführen, Bild 2.19. Auch stellt der Radius $r=1,0 \mathrm{~mm}$ einen groben Mittelwert für real gemessenen Kerbradien an Schweißverbindungen dar und wurde bereits bei Radaj eingeführt. Auf die Begründung mit der Mikrostützwirkung wird gänzlich verzichtet.

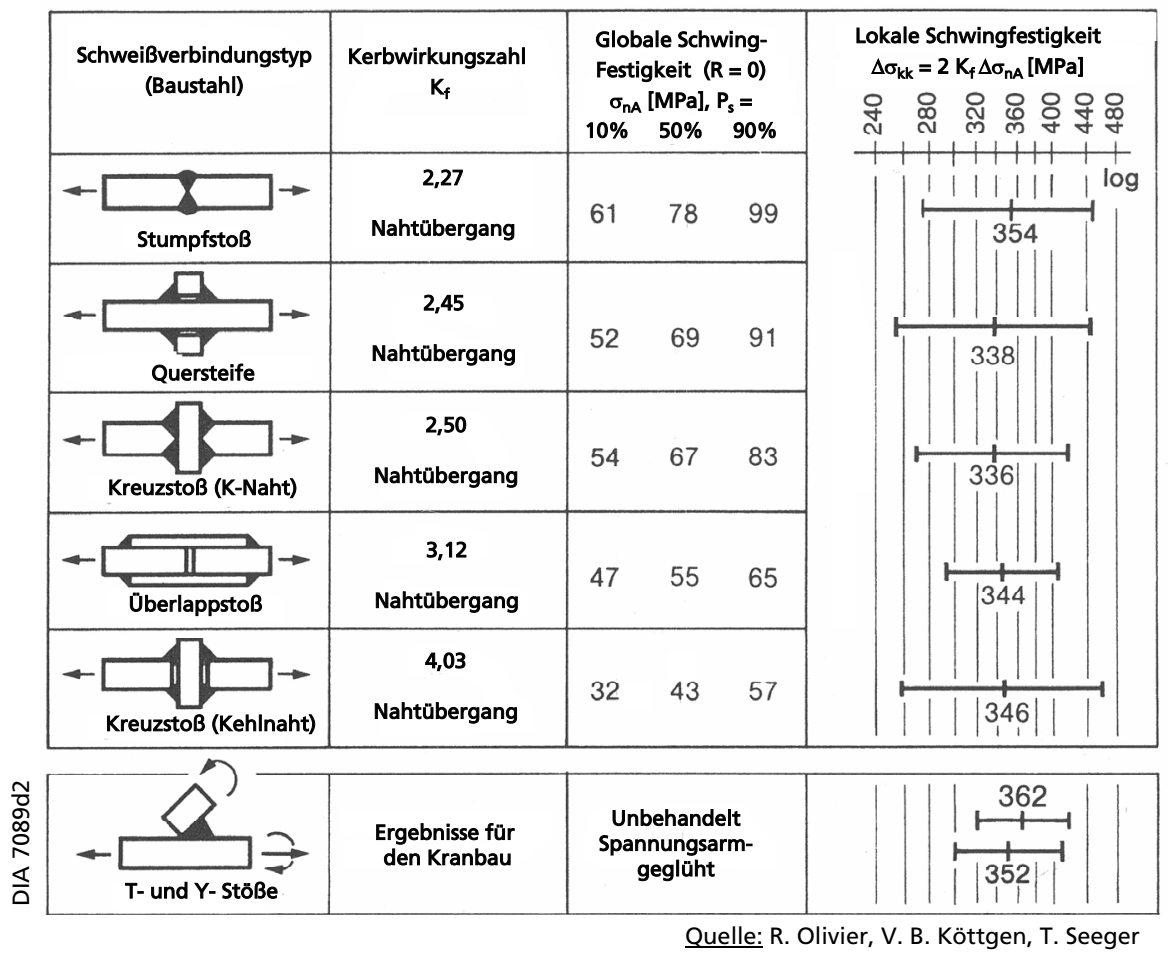

Bild 2.19: Ertragbare Spannungen beim Ersatzradius $r_{f}=1,0 \mathrm{~mm}$ für Baustahl

Für Stahl konnte zu Bemessungszwecken eine Masterwöhlerlinie ermittelt werden, die in [Oli94] als zulässige Kerbgrundspannung mit $\Delta \sigma=225 \mathrm{MPa}$ bei der Schwingspielzahl $\mathrm{N}=2 \cdot 10^{6}$ angegeben ist und damit der FAT-Klasse 225 entspricht. Der gleiche fiktive Radius ergibt sich aber auch für geschweißte Verbindungen aus Stahl nach der Theorie der Mikrostützwirkung aus der Beziehung (Gl. 2.21) mit dem Mehrachsigkeitsfaktor $s=2,5$ (Hypothese v. Mises) und der Ersatzstrukturlänge $\rho^{\star}=0,4 \mathrm{~mm}$.

Das Konzept des fiktiven Ersatzradius konnte auch schon bei punktgeschweißten und später laserstrahlgeschweißten Dünnblechverbindungen mit $\mathrm{t} \leq 3 \mathrm{~mm}$ bei Aluminium und Stahl angewendet werden, allerdings mit einem Ersatzradius von $r_{f}=0,05 \mathrm{~mm}$ [Eib03-2, Eib03, Eib01, Schl03, Zha02]. Die Übertragung auf dickwandige Aluminiumschweißverbindungen ist Thema dieser Arbeit.

In Gegensatz zum Konzept der Mikrostützwirkung nach Radaj wird bei dem Konzept des fiktiven Krümmungsradius dieser nicht werkstoffmechanisch abgeleitet, sondern aus Schwingfestigkeitsergebnissen mit unterschiedlich stark gekerbten Schweißverbindungen abgeleitet. Es wird der Radius gesucht, mit dem alle Kerben der Schweißverbindungen fiktiv 
gerundet werden können, und bei der Auftragung im lokalen Spannungssystem zu einer minimalen Streuung führt - Mittelwertstreuungskonzept. Mit dem gefundenen fiktiven Ersatzradius können in gewissen Werkstoffdickenbereichen alle Arten von Schweißverbindungen über einen Vergleich mit einer Master-Wöhlerlinie im lokalen Spannungssystem bewertet werden. Für Stahlschweißverbindungen findet sich dieses vorgehen bereits in Regelwerken [FKM03, Hob03]. Bei Aluminiumschweißverbindungen liegen auch erste Ansätze zur Anwendung vor [Eib03-2, Grz03].

\section{$2.4 \quad$ Werkstoffvolumenkonzept}

Das Werkstoffvolumenkonzept besagt, dass die Schwingfestigkeit bis zum Anriss um so größer ist, je kleiner das höchstbeanspruchte Werkstoffvolumen ist [Son93]. Für die Berechnung des höchstbeanspruchten Werkstoffvolumens wird auf Grund von Erfahrungen der Bereich, in dem mehr als $90 \%$ der maximalen Hauptspannung herrschen, als das höchstbeanspruchte Volumen $\mathrm{V}_{90 \%}$ definiert, Bild 2.20.

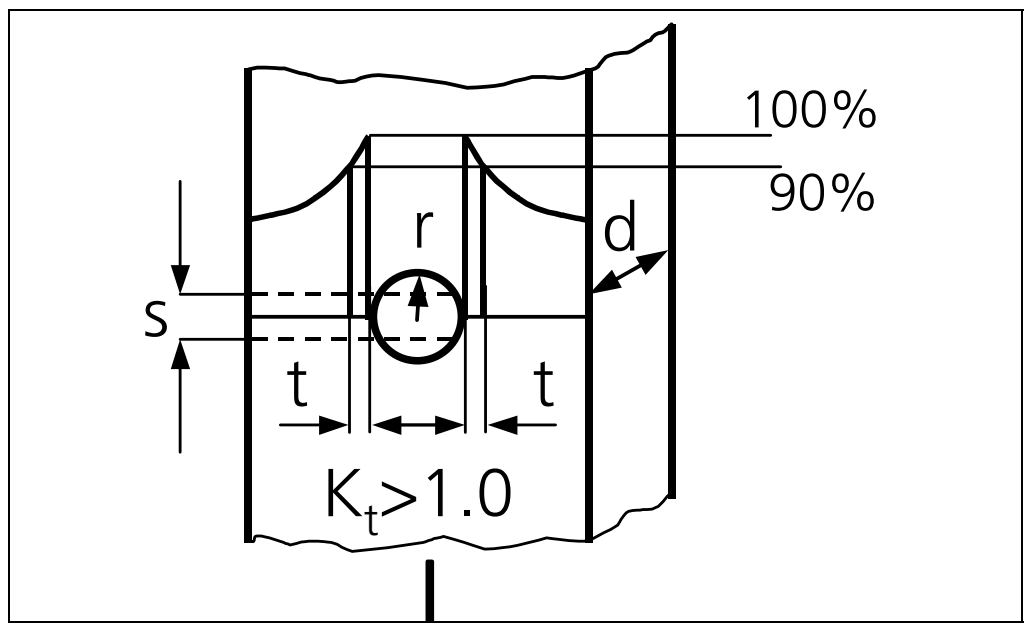

Bild 2.20: Definition des höchstbeanspruchten Werkstoffvolumens $V_{90}$

Dieses Volumen lässt sich bei komplexen Geometrien mit einer Finite-Elemente Berechnung anhand der ermittelten Dehnungs- bzw. Spannungsverteilung bestimmen. Bei relativ einfachen Geometrien kann dies je nach Belastungsart über das bezogene Spannungsgefälle der Hauptspannung

$\chi^{*}=\frac{\mathrm{d} \sigma_{1}}{\mathrm{dt}} \cdot \frac{1}{\sigma_{1, \max }}$,

dass ein Maß für die Stützwirkung ist, festgelegt werden. Aus der Tiefe, in der das Hauptspannungsmaximum um 10\% abfällt,

$\mathrm{t}_{10 \%}=\frac{0,10}{\chi^{*}}$

und den entsprechenden Abmessungen entlang der Oberfläche, an der die Spannung ebenfalls um $10 \%$ abnimmt, ist das jeweilige höchstbeanspruchte Volumen $V_{90 \%}$ berechenbar. 


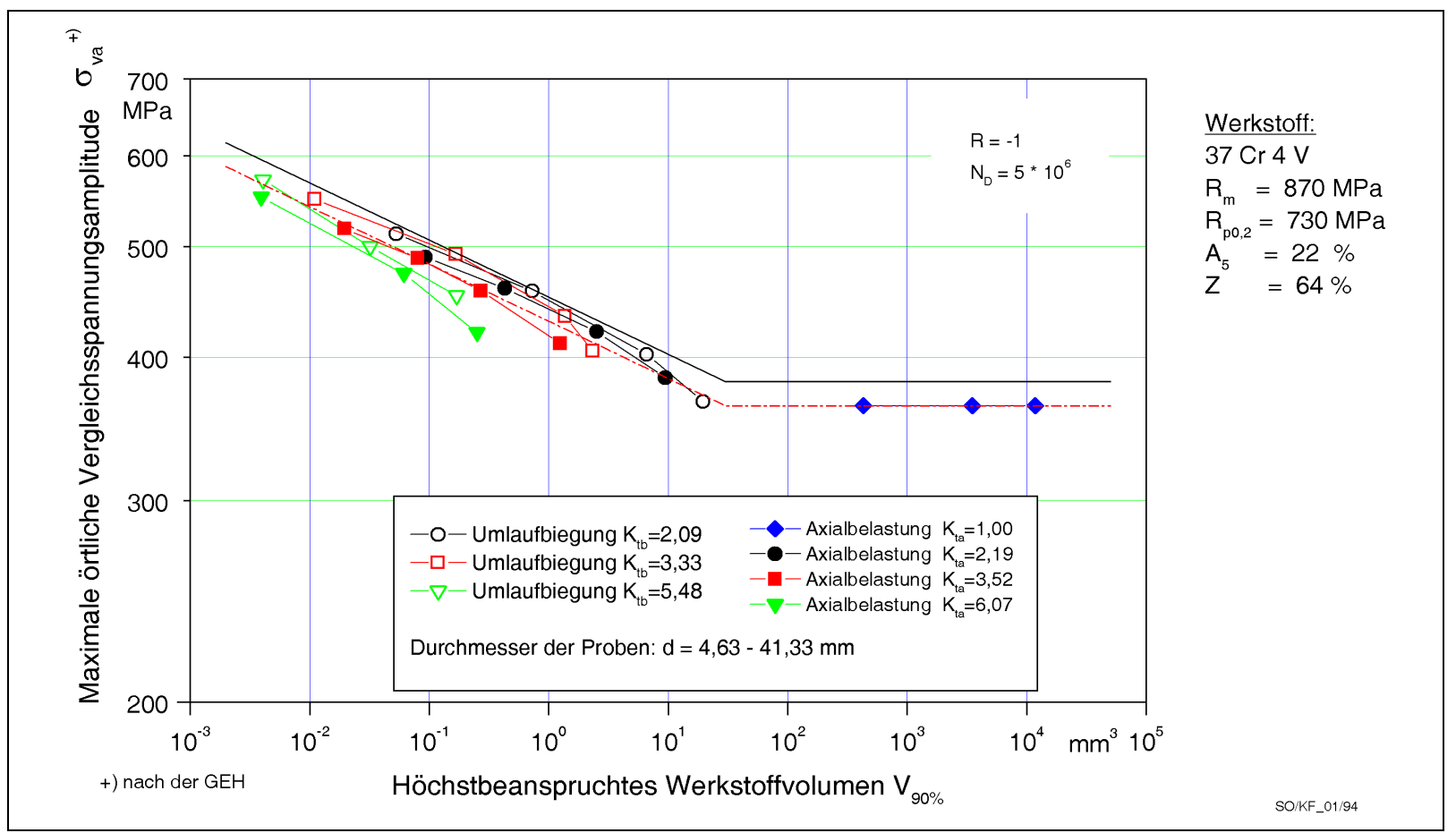

Bild 2.21: Werkstoffvolumenkonzept

In diesem Konzept werden die Einflüsse aus dem Größeneinfluss und dem Gradienteneinfluss an Kerbstellen gemeinsam berücksichtigt und können in einem mit FE-Methoden leicht zu berechnenden Wert abgebildet werden.

Über einen werkstoffspezifischen funktionalen Zusammenhang zwischen höchstbeanspruchten Werkstoffvolumen $V_{90}$ und schwingfest ertragbarer Spannung kann für einen konkreten Beanspruchungsfall die zulässige Spannung berechnet werden, Bild 2.21.

\subsection{Bruchmechanisches Konzept}

Das Bruchmechanikkonzept kann auf verschiedene Art und Weise angewendet werden [Hob05, Son05-3]. Es kann zur Beschreibung einer Spannungskonzentration an einer scharfen Kerbe, einem Wurzelspalt oder einer Unregelmäßigkeit durch eine Spannungsintensität und darüber hinaus auch für Rissfortschrittsberechnungen herangezogen werden.

Im Falle einer Festigkeitsbeurteilung anhand einer Spannungsintensität muss zur Bewertung eine Spannungsintensitätswöhlerlinie vorliegen, die nur durch entsprechende Versuche abgeleitet werden kann.

\subsubsection{Rißfortschrittskonzept}

Die Bruchmechanik erweist sich insbesondere bei sicherheitsrelevanten Schweißverbindungen als ein nützliches Werkzeug zur Bewertung von Unregelmäßigkeiten, sofern diese durch entsprechende Detektionsmethoden zutreffend erfasst werden, sowie zur Festlegung von Inspektionsintervallen bzw. Planung von Reparaturmaßnahmen anhand von Rissfortschrittsberechnungen.

Die Rissausbreitung lässt sich in Stadium I und II gliedern. In der Regel entstehen Ermüdungsrisse an der Werkstoffoberfläche. Allerdings können sich Risse bei starken Inhomogenitäten, wie z.B. grobe Einschlüsse, Lunker und gehärteten Oberflächenschichten auch unterhalb der Oberfläche 
bilden. Der für viele metallische Werkstoffe typische und bekannteste Vorgang ist die Rissbildung an Ermüdungsgleitbändern. Diese geht kontinuierlich in die erste Phase der Rissausbreitung Stadium I - über, in der sich der Riss längs den Gleitebenen von der Oberfläche ausgehend in das Probeninnere ausbreitet. Da nur die schubspannungsmäßig günstig orientierten Gleitsysteme Ermüdungsgleitbänder erzeugen, treten die Gleitbandrisse nahe der Ebene der größten Schubspannung auf. Nachdem ein bis mehrere Körner auf diese Weise durchquert sind, beginnt meist das Stadium II der Rissausbreitung. Der Riß folgt nun einer makroskopischen, nicht kristallographisch definierten Ebene, die senkrecht zur angelegten Spannung liegt. Ein charakteristisches Merkmal der Ermüdungsbruchfläche im Stadium II stellen die als Riefen senkrecht zur Rissausbreitung verlaufenden Schwingungsstreifen dar. Diese treten bei Rissgeschwindigkeiten größer als $10^{-5} \mathrm{~mm} / \mathrm{Schwingspiel}$ auf und werden bei Aluminiumlegierungen bevorzugt beobachtet [Gro71].

Bei der Untersuchung der Rissausbreitung wird allgemein die Risslänge a als Funktion der Schwingspielzahl N gemessen und die Rissfortschrittsgeschwindigkeit ermittelt. Die Korrelation der Rissgeschwindigkeit mit der Spannungsintensität K liegt der Vorstellung zugrunde, dass die Ausbreitung von Ermüdungsrissen im wesentlichen von zyklischen plastischen Deformationen an der Rissspitze bestimmt wird, die von der zyklischen Spannungsintensität $\Delta \mathrm{K}$ gesteuert werden. Die zyklische Spannungsintensität wird unter Verwendung des Geometriefaktors $F$ für die jeweilige Proben- und Rissgeometrie, wie folgt, beschrieben:

$\Delta \mathrm{K}=\Delta \sigma \cdot \sqrt{\pi \cdot a} \cdot \mathrm{F}$.

Bruch tritt ein, wenn die zyklische Spannungsintensität $\Delta K_{c}$ erreicht ist.

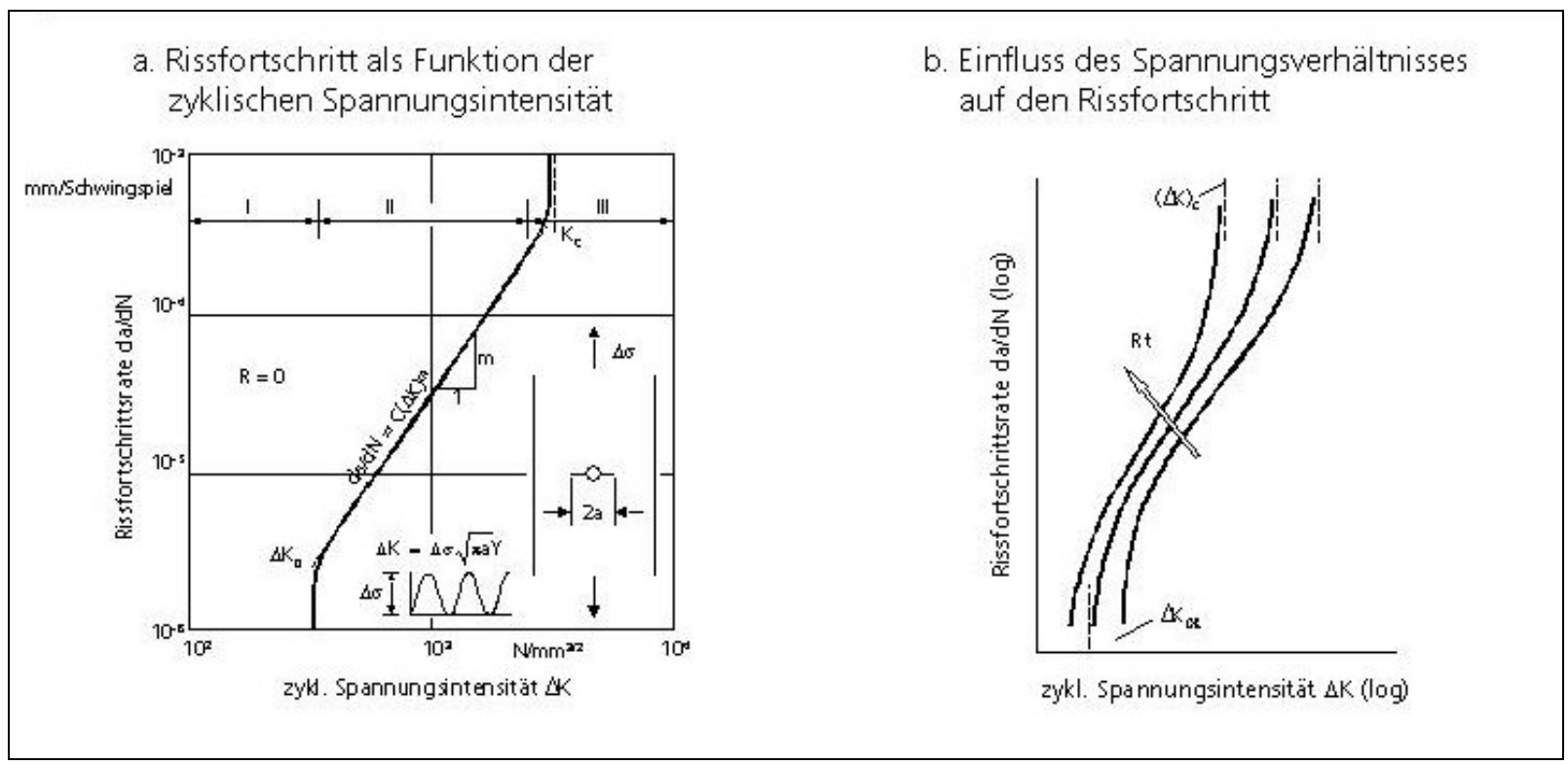

Bild 2.22: Bruchmechanisches Konzept

Bei der allgemein üblichen Auftragung von da/dN über $\Delta \mathrm{K}$ im doppeltlogarithmischen Koordinatensystem ergibt sich der in Bild 2.22 gezeigte S-förmige Kurvenverlauf. Im Bereich niedriger Spannungsintensität (Bereich I) nähert sich die Kurve dem Grenzwert $\Delta K_{0}$, dem Schwellenwert der Spannungsintensität, an. Ist die zyklische Spannungsintensität kleiner als $\Delta \mathrm{K}_{0}$ findet kein Risswachstum statt. Im Bereich II, in dem der überwiegende Teil der Rissfortschrittsuntersuchungen durchgeführt wird, stellt sich ein linearer Zusammenhang zwischen $\log (\mathrm{da} / \mathrm{dN})$ und $\log (\Delta \mathrm{K})$ ein, der durch die Paris-Gleichung beschrieben werden kann [Par61]: 
$\frac{\mathrm{da}}{\mathrm{dN}}=\mathrm{C} \cdot \Delta \mathrm{K}^{\mathrm{m}}$

Die Konstanten $\mathrm{C}$ und $\mathrm{m}$ sind werkstoffabhängig. An den Bereich $\|$ grenzt der instabile Restbruch, der nach dem Erreichen der kritischen Spannungsintensität $\mathrm{K}_{c}$ im Bereich III eintritt.

Der Einfluss des Spannungsverhältnisses R

$R=\frac{\sigma_{\min }}{\sigma_{\max }}=\frac{K_{\min }}{K_{\max }}$

auf die Rissfortschrittsrate ist in Bild Bild 2.22 schematisch eingetragen. Die Kurve verschiebt sich mit zunehmenden $\mathrm{R}$ nach links. Die Rissfortschrittsgeschwindigkeit nimmt in dieser Richtung zu. Entsprechend verändern sich $\Delta \mathrm{K}_{0}$ und $\Delta \mathrm{K}_{\mathrm{c}}$. Für den mittleren Bereich kann der Einfluss des Spannungsverhältnisses durch die Gleichung nach Forman beschrieben werden [For67]:

$$
\frac{d a}{d N}=\frac{C^{\prime}(\Delta K)^{m}}{(1-R) \cdot K_{c}-\Delta K}=\frac{C^{\prime}(\Delta K)^{m}}{(1-R) \cdot\left(K_{c}-K_{\max }\right)} .
$$

Zahlreiche Untersuchungen [Sch81, New84, Kem89, Vor91, Fin92, Sav94] zeigen, dass der Riss insbesondere unter Druckbeanspruchung schließt und erst ab einer definierten Spannung, der so genannten Rissöffnungsspannung, wieder öffnet, um weiter fortzuschreiten. Die Rissöffnungsspannung hängt dabei vom Spannungsverhältnis $R$ und der Höhe des Beanspruchungshorizontes $a b$. Der Rissfortschritt wird nicht mehr von $\Delta K$, in welche die Gesamtspannungsschwingbreite $\Delta \sigma$ eingeht, sondern vom Effektivwert $\Delta K_{\text {eff, gesteuert. Der }}$ große Einfluss des Rissschließens auf die Schwingfestigkeit bzw. die Lebensdauer unter variabler Amplitude wird ausführlich in [Elb70, Elb71] beschrieben.

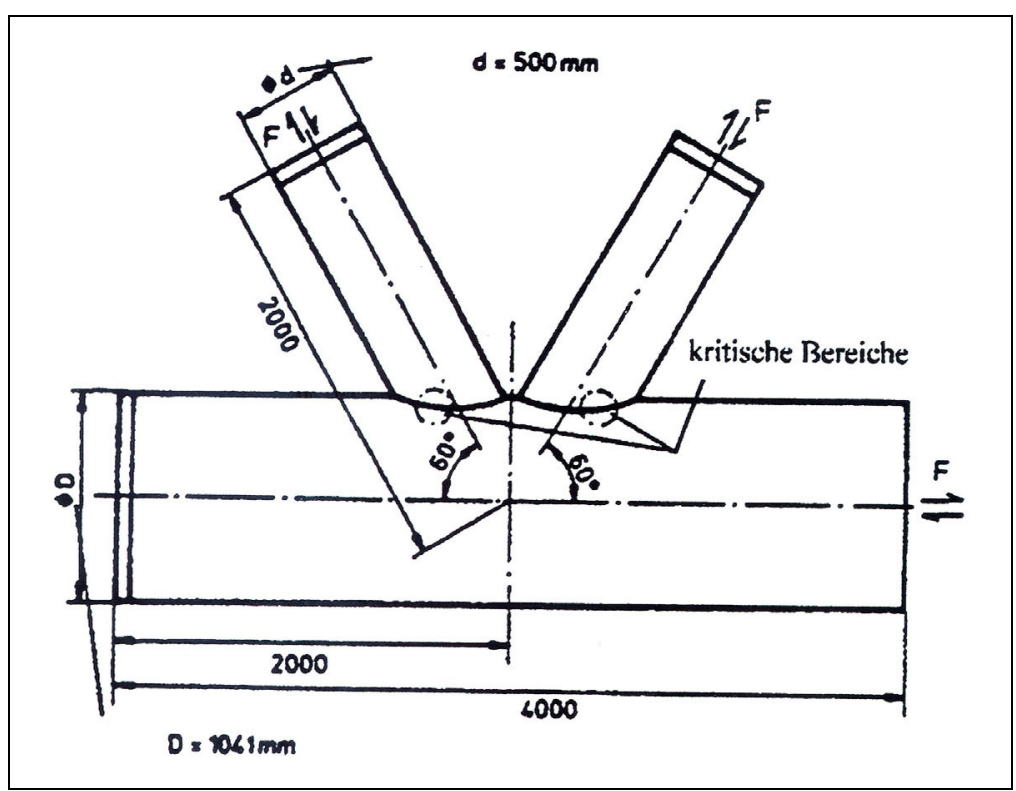

Bild 2.23: Rohrknoten mit anrisskritischen Bereichen in der Nahtübergangskerbe 
Die zyklische Spannungsintensität wird unter der Bedingung, dass die plastischen Zonen an der Rissspitze klein gegenüber der Risslänge ist, angewandt. Bei größerer plastischer Zone an der Rissfront sind die Voraussetzungen der linear-elastischen Bruchmechanik nicht mehr erfüllt. Anstelle der zyklischen Spannungsintensität $\Delta \mathrm{K}$ tritt das zyklischen J-Integral $\Delta \mathrm{J}$.

Beispiele für die Anwendung des Rissfortschrittskonzeptes finden sich zahlreich in der FKMRichtlinie zum bruchmechanischen Festigkeitsnachweis [FKM02]. Dabei wird insbesondere auf die Anwendung in der Qualitätskontrolle eingegangen, bei der bestimmte Risslängen, die sich bei regelmäßigen Inspektionen mit zerstörungsfreien Prüfverfahren detektieren lassen, angenommen werden, z.B. bei Rohrknoten an seegangsbelasteten Offshorekonstruktionen, Bild 2.23 . Die Abschätzung des Rissfortschrittes durch den Vergleich von Rechnung und Experiment kann die zur Bewertung der Restlebensdauer herangezogen werden, Bild 2.24.

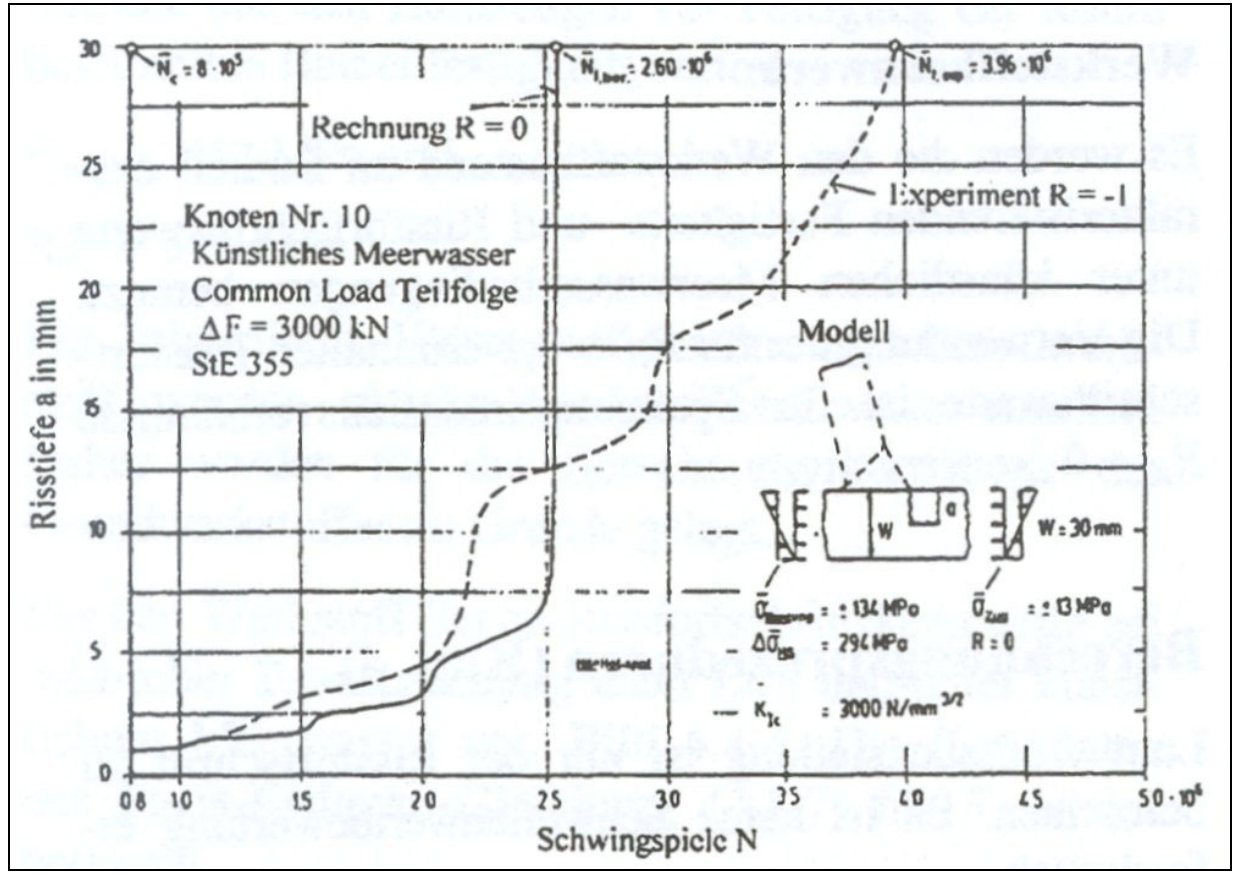

Bild 2.24: Vergleich des Rissfortschritt in Experiment und Rechnung

\subsubsection{Spannungsintensitätskonzept}

Der Spannungszustand mit Singularität an scharfen einspringenden Eckkerben (V-Kerben) lässt sich durch Kerbspannungsintensitätsfaktoren (notch stress intensity factors NSIFs) beschreiben [Liv04]. Dabei erscheint die rissartige Kerbe als Sonderfall mit gegen Null gehendem Kerböffnungswinkel. Diese Beschreibung gilt exakt bei verschwindendem Kerbrundungsradius, aber auch noch näherungsweise bei kleinem Radius, und zwar in kleinem Abstand vom Kerbgrund. Der Übergang zur Kehlnaht stellt sich als eine Kerbe mit etwa $135^{\circ}$ Öffnungswinkel dar, mit sehr kleinem oder gar verschwindendem Rundungsradius, Bild 2.25. Das lokale Spannungsfeld am Nahtübergang lässt sich daher durch die Angabe des NSIF eindeutig beschreiben. Er hängt ab von der Strukturspannung am Übergang (Membran- und Biegeanteil), von den Lastableitungsbedingungen und von der Plattendicke (Größeneffekt). Es wird zwischen den Grundbeanspruchungsmoden I, II und III unterschieden, die sich überlagern. 


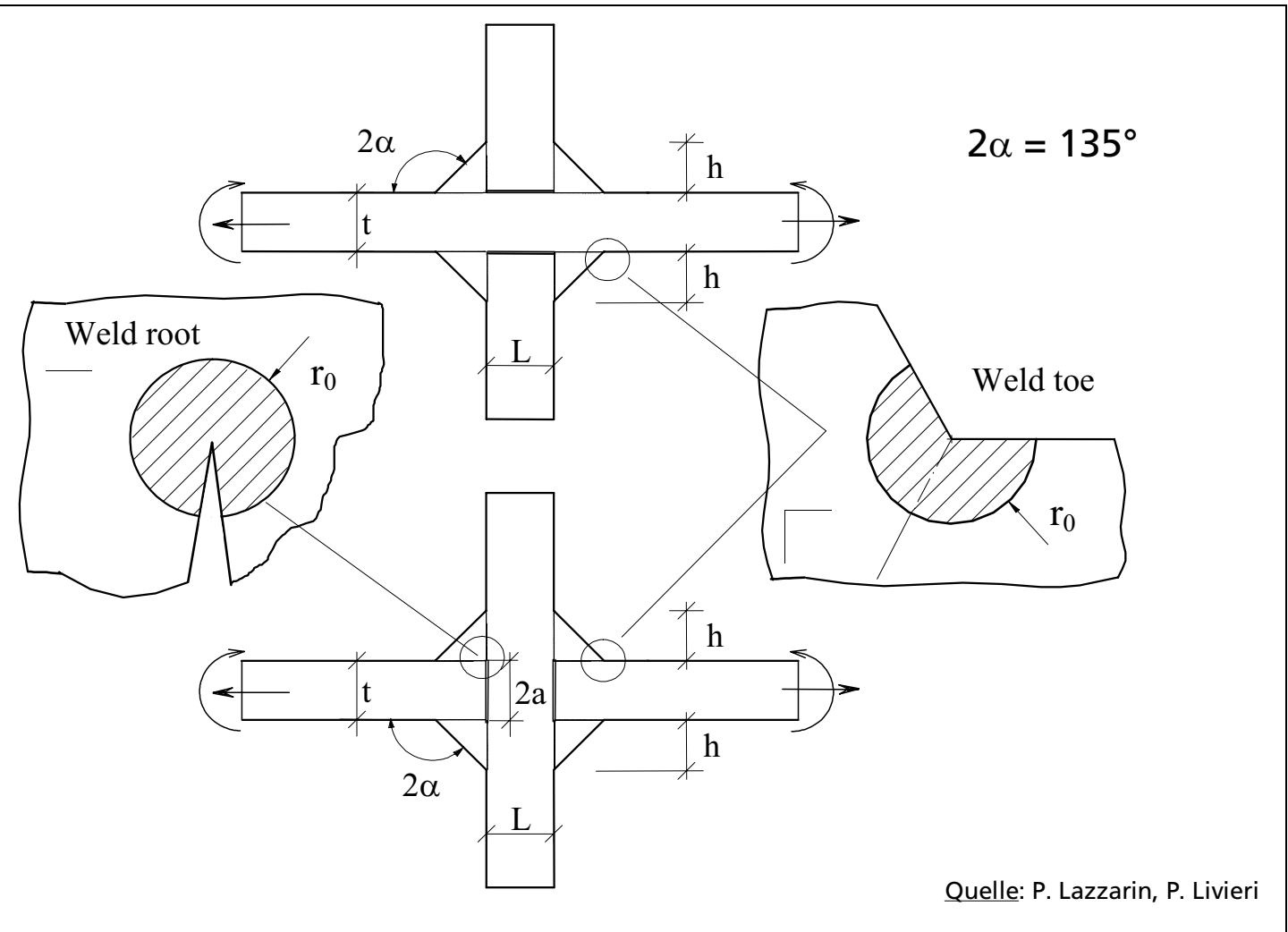

Bild 2.25: Veranschaulichung der Sektorflächen

In der gängigen Kreuzstoß- oder Überlappprobe mit Kehlnähten unter Zug- oder Biegebelastung ist i. a. allein der Beanspruchungsmodus I maßgebend (Modus II ist bei $135^{\circ}$ Kerböffnungswinkel nichtsingulär). Es ist zu erwarten, dass die Ermüdungsfestigkeit der Proben dominant von der Schwingbreite des NSIF abhängt. Die sich im Wöhler-Diagramm ergebenden Streubänder der

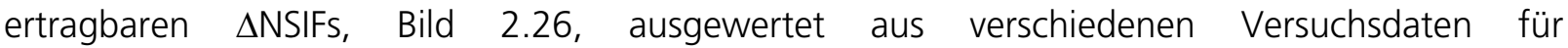
nichtlasttragende Kehlnähte an Proben aus Stählen und Aluminiumlegierungen, sind im Hinblick auf den erfassten Dickenbereich hinreichend eng. Der Dickeneffekt ist eingeschlossen: $t=13-100$ $\mathrm{mm}$ bei den Stählen und $\mathrm{t}=3-24 \mathrm{~mm}$ bei den Aluminiumlegierungen. Er wird durch den theoretischen Exponenten $\left(1-\lambda_{1}=0,326\right)$ gekennzeichnet - zu vergleichen mit dem Exponenten 0,1 $-0,3$ in bekannten Regelwerken. Das NSIF-Konzept gilt primär für die Risseinleitung $\left(a_{\mathrm{i}} \approx 0,3 \mathrm{~mm}\right)$, ist aber näherungsweise auf obige Versuchsdaten übertragbar. 


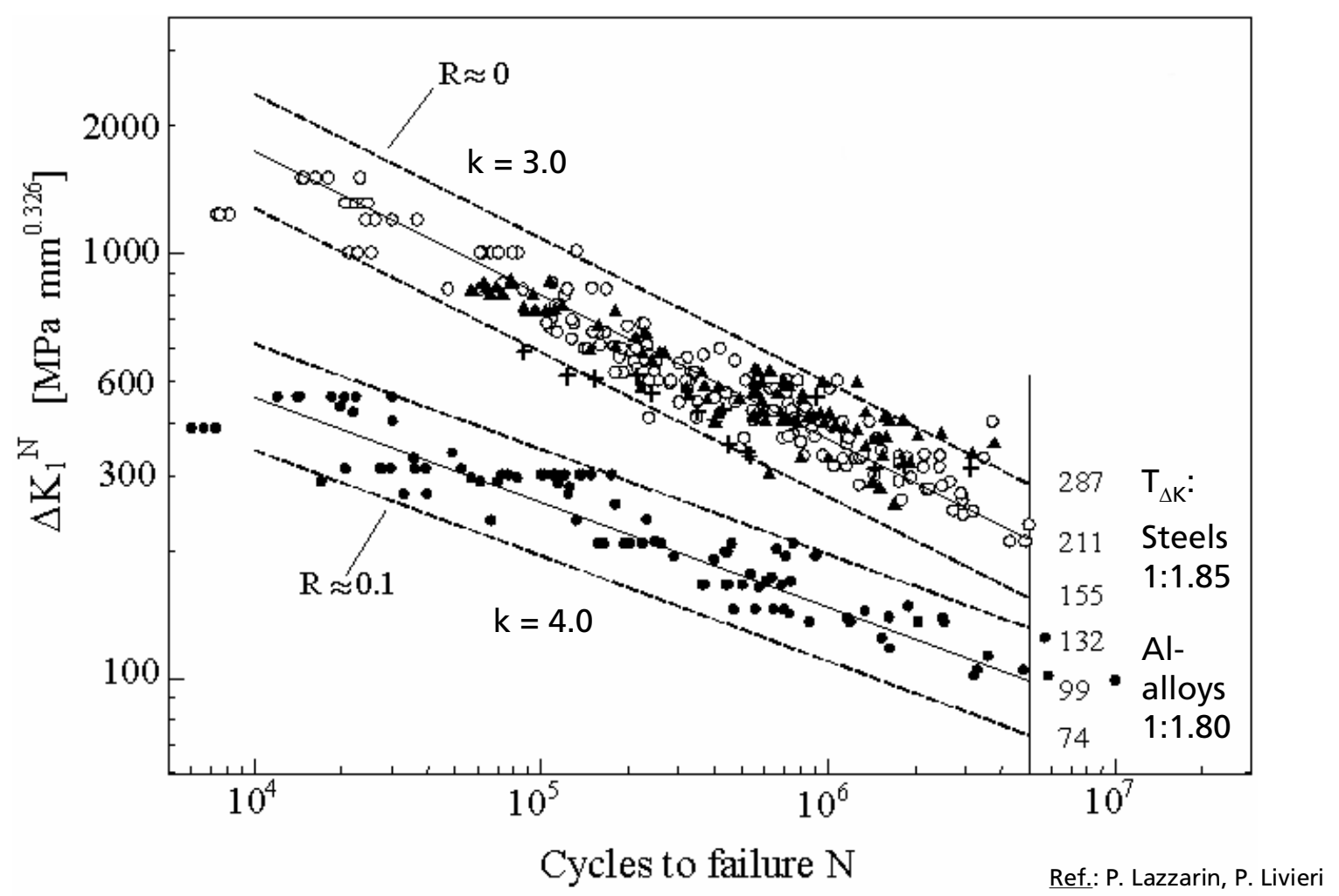

Bild 2.26: Ergebnisse nach dem Kerbspannungsintensitätskonzept für kehlnaht-geschweißte Quersteifen unter Axial- und Biegebelastung

Bei der Anwendung des NSIF-Konzepts ergeben sich dadurch Schwierigkeiten, dass die Dimension des NSIF vom Kerböffnungswinkel abhängt [Hen01]. Beispielsweise lassen sich die Spannungsintensitätsfaktoren von Nahtübergang $\left(135^{\circ}\right)$ und Nahtwurzel $\left(0^{\circ}\right)$ nicht direkt vergleichen. Abhilfe wurde durch Erweiterung des Konzepts auf das Kriterium der über einen kleinen Bereich an der Kerb- oder Rissspitze gemittelten Formänderungsenergiedichte geschaffen. Dieser Ansatz erlaubt es außerdem, den gemischten Beanspruchungsmodus, das lokale Fließen und die Rundung der Rissspitze in ihrer Auswirkung auf die Ermüdungsfestigkeit zu erfassen.

Das NSIF-Konzept (detailliertere Übersicht in [Rad05]) hat somit einen Entwicklungsstand erreicht, der sich für die praktische Anwendung anbietet, selbstverständlich nach weiterer Erprobung, insbesondere auch an Bauteilen. Eine weitere Einengung der Streubereiche ist anzustreben.

\subsection{Schadensparameter}

Durch die Einführung von Schadensparametern ist es insbesondere bei elastoplastischen Beanspruchungen möglich, neben der Spannungsamplitude auch auftretende Mittelspannungen und Mitteldehnungen bei der Berechnung der Schwingfestigkeit zu berücksichtigen, [Bes91, Hai76]. Verbreitet ist der Schadensparameter nach Smith, Watson und Topper - $P_{\text {SWT }}[$ [Smi70]:

$P_{S W T}=\sqrt{\left(\sigma_{m}+\sigma_{a}\right) \cdot \varepsilon_{a} \cdot E}$. 
Dieser berücksichtig das lokale Fließverhalten des Werkstoffe sowie den Einfluss der vorhandenen Mittelspannung auf die Lebensdauer; dies ist das am häufigsten angewandte Verfahren der Amplitudentransformation bei der Anwendung örtlicher Konzepte. Darüber hinaus kann es sinnvoll sein, bei großen Mitteldehnungen den Schadensparameter $P_{\varepsilon}$ zu verwendet,[Wer99-2]:

$P_{\varepsilon}=\sqrt{\left(\varepsilon_{m}+\varepsilon_{a}\right) \cdot \sigma_{a} \cdot E}$.

Dieser führt im Vergleich zum Parameter $\mathrm{P}_{\mathrm{sWT}} \mathrm{zu}$ besseren Ergebnissen, da hier die Dehnungen im Vergleich zu den Spannungen stärker gewichtet werden. Auch gibt es Schadensparameter, die bruchmechanische Kennwerte mit einbeziehen, wie der Parameter P, nach [Vor89].

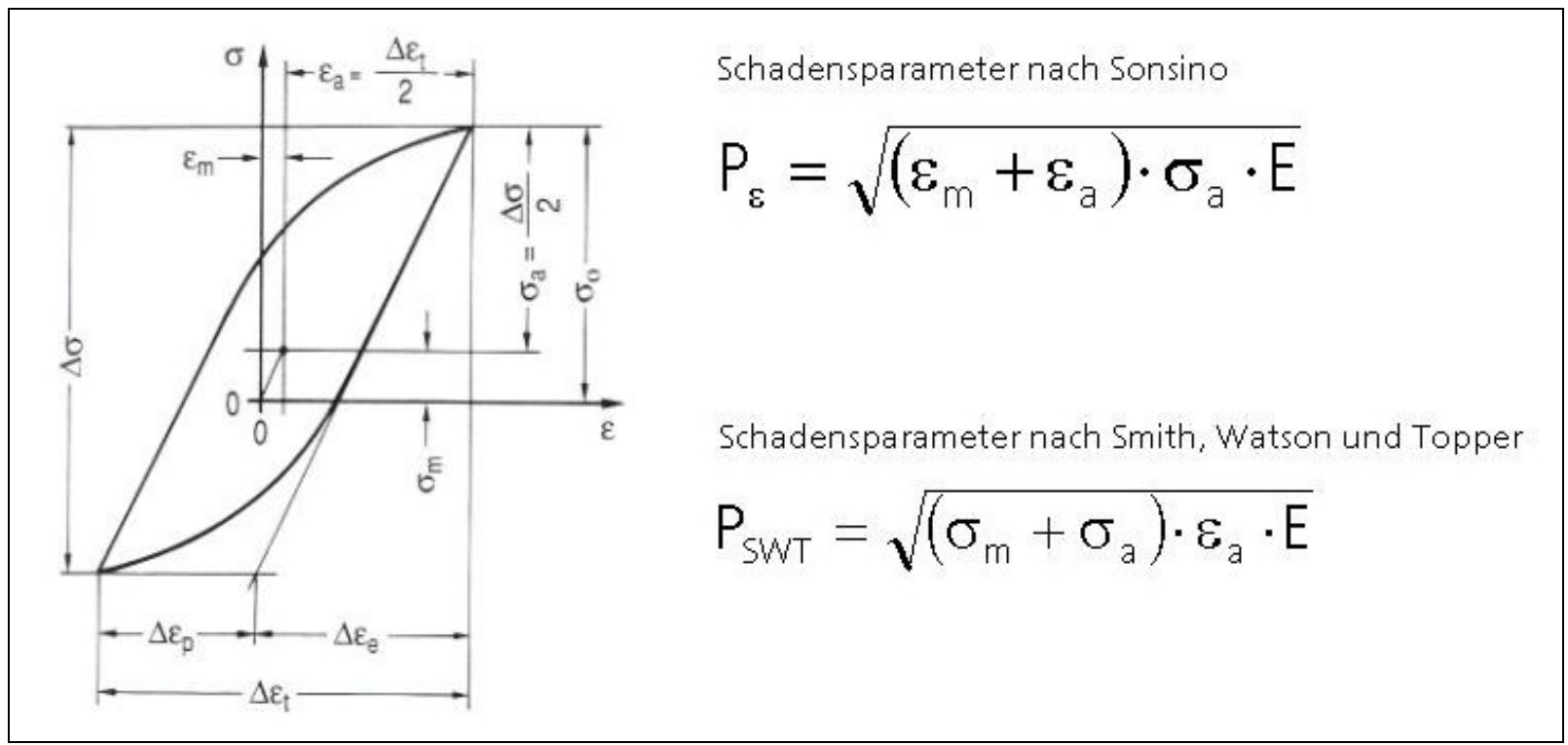

Bild 2.27: Berechnung der Schadensparameter $P_{S W T}$ und $P_{\varepsilon}$

Allerdings können, je nach Werkstoff, mehr oder weniger große Unterschiede in der Bewertung des Mittelspannungseinfluss nach den P-Konzepten und Mittelspannungsempfindlichkeiten mittels eines Haigh-Diagramms auftreten [Bux92, Hai02, Schü73].

\subsection{Lebensdauerabschätzung bei veränderlichen Betriebsbelastungen}

Die Schadensakkumulation ermöglicht den Übergang von der Lebensdauerabschätzung unter Belastung mit konstanten Amplituden zu der Lebensdauerabschätzung mit variablen Amplituden und kann prinzipiell bei allen zuvor dargestellten Konzepten angewendet werden. Dies bedeutet, dass tatsächlich im Betrieb auftretende unregelmäßige Belastungen berücksichtigt werden können. Bei der Schadensakkumulation wird jedem Schwingspiel bzw. der Anzahl der Schwingspiele auf einem Lasthorizont $n_{i}$ eine Teilschädigung $D_{i}$ zugewiesen, indem $n_{i}$ der auf diesem Belastungshorizont im Wöhlerversuch ertragbaren Schwingspielzahl $N_{i}$ gegenübergestellt wird. Die Einzelschädigungen werden zur Schadenssumme D aufaddiert, Bild 2.28. 


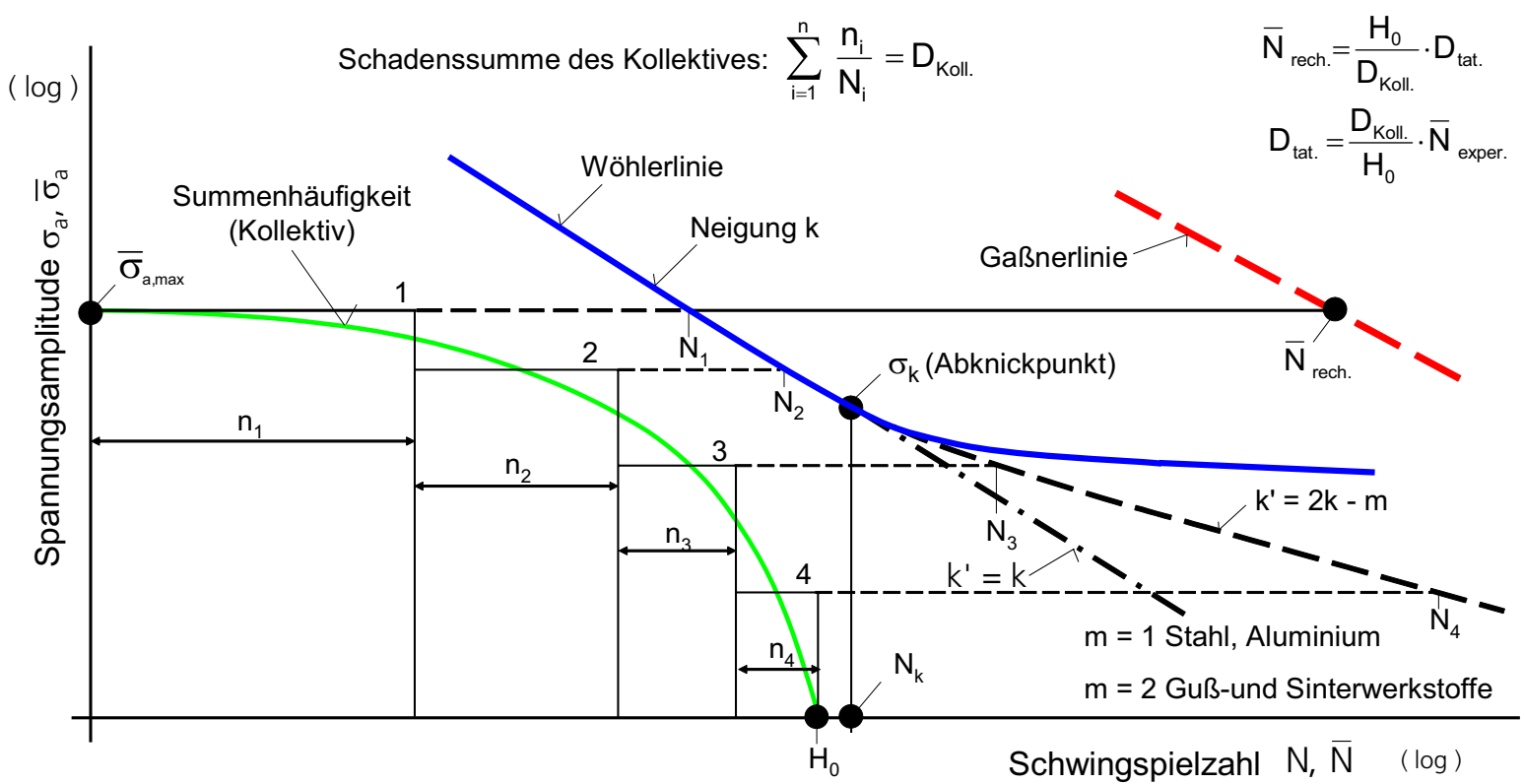

Bild 2.28: Berechnung der Lebensdauer (schematisch)

Ist die Schadenssummen $\mathrm{D}=1$ erreicht, tritt theoretisch das Versagen ein [Min45].

$D=\sum_{i} D_{i}=\sum_{i} \frac{n_{i}}{N_{i}}=1$

In der Praxis hat sich jedoch gezeigt, dass die tatsächliche Schadenssumme Werte zwischen 0,1 und 10 sowie darüber hinaus annehmen kann [Eul97-1, Eul99], Bild 2.28..

Deshalb wird aufgrund von Erfahrungswerten oder Experimenten für vergleichbare Belastungsverteilungen und vergleichbare Kerben eine zulässige Schadenssumme $D_{\text {tat }}$ festgelegt, die größer oder kleiner eins sein kann. Dies wird als Relativ-Miner-Regel bezeichnet [Low73, Schü73, Buc83, Son97-1, Son97-2, Gru88, Hai89, Bra95]:

$D_{\text {tat }}=\frac{D_{i}}{H_{T F}} \cdot \bar{N}_{\text {exp }}$ 


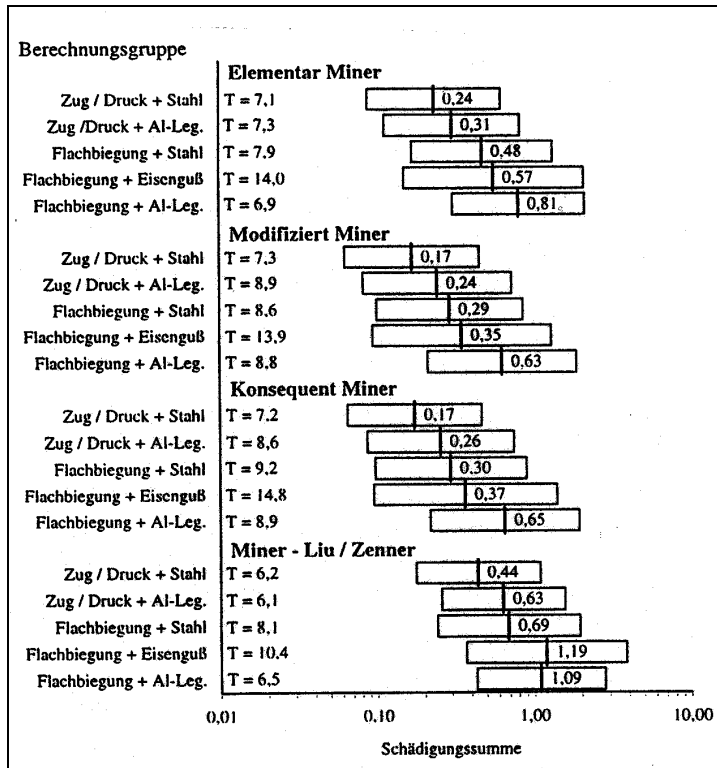

nach K.-G. Eulitz und K.L. Kotte

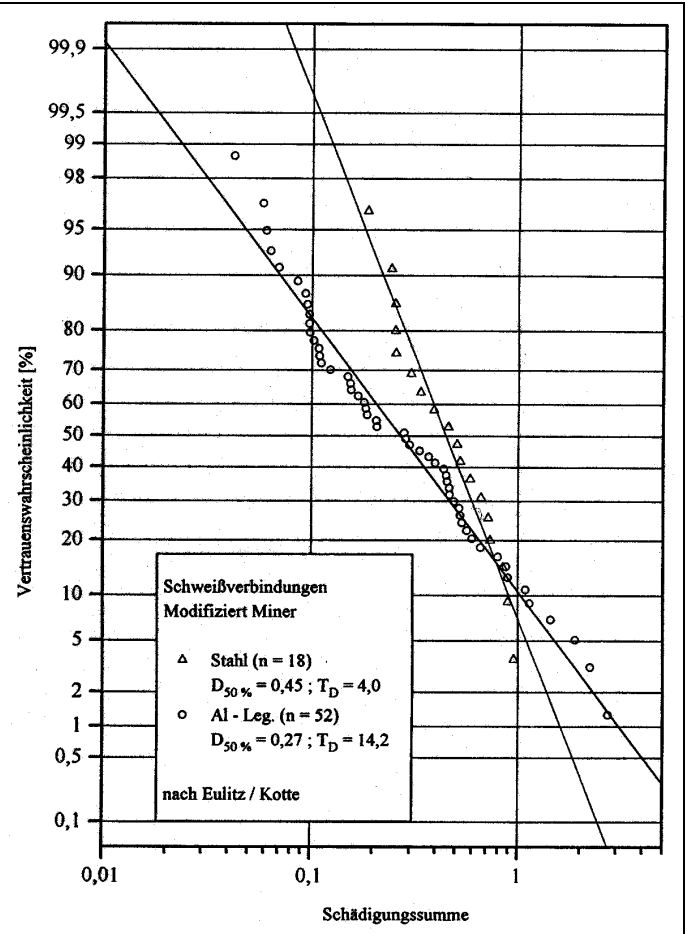

Bild 2.29: Mittelwerte und Streuung der Schadenssumme

Als $\mathrm{H}_{\mathrm{TF}}$ wird der Teilfolgenumfang bezeichnet; $\mathrm{N}_{\exp }$ stellt die im Schwingfestigkeitsversuch unter variabler Amplitude experimentell ermittelte Lebensdauer dar.

Nach der orginalen Miner-Regel bewirken Schwingspiele mit Beanspruchungen kleiner als die so genannte Dauerfestigkeit (Spannung unterhalb des Knickpunktes der Wöhlerlinie) keine Schädigungen, Bild 2.28. Es hat sich herausgestellt, dass dies nicht der Wirklichkeit entspricht. Zu der ursprünglich von Palmgren und Miner entwickelten linearen Schadensakkumulationshypothese existieren deshalb zahlreiche varianten und Weiterentwicklungen [Hai70, Fat98, Zen92, Bru90, Dow88], die das tatsächliche Werkstoffverhalten besser abbilden sollen, insbesondere, wenn nach dem Abknickpunkt auch bei hohen Schwingspielzahlen mit einem weiteren Abfall der Schwingfestigkeit zu rechnen ist.

Zur Erfassung der Schädigung von Amplituden unterhalb der so genannten Dauerfestigkeit können die Modifikationen nach Haibach, nach Corten und Dolan sowie nach Liu und Zenner angewandt werden [Zen92]. Bei dem von Haibach und Corten-Dolan vorgeschlagenen Verfahren wird oberhalb der so genannten Dauerfestigkeit gegen die ursprüngliche Wöhlerlinie gerechnet. Unterhalb der Dauerfestigkeit wird nach Haibach für die Schädigungsrechnung eine Wöhlerlinie mit einer Neigung von

$k^{\prime}=2 k-1 \quad$ für Stahl und Aluminium (geschmiedet, gewalzt, geknetet)

$k^{\prime}=2 k-2 \quad$ für den Schweiß- oder Gusszustand

angenommen, während nach Corten und Dolan die originäre Wöhlerlinie unterhalb der Dauerfestigkeit mit konstanter Neigung weiterläuft, es also keinen Abknickpunkt gibt. Diese Variante wird auch als Miner-Elementar bezeichnet. Bei der Modifikation nach Liu und Zenner wird die ursprüngliche Wöhlerlinie um den Kollektivhöchstwert gedreht, Bild 2.30. Die neue Neigung ist der Mittelwert aus der ursprünglichen Neigung und der Neigung der Rissfortschrittswöhlerlinie. Die für diese Rechnung zugrunde gelegte Wöhlerlinie knickt erst auf der Höhe der halben „Dauerfestigkeit” in die Horizontale ab. 


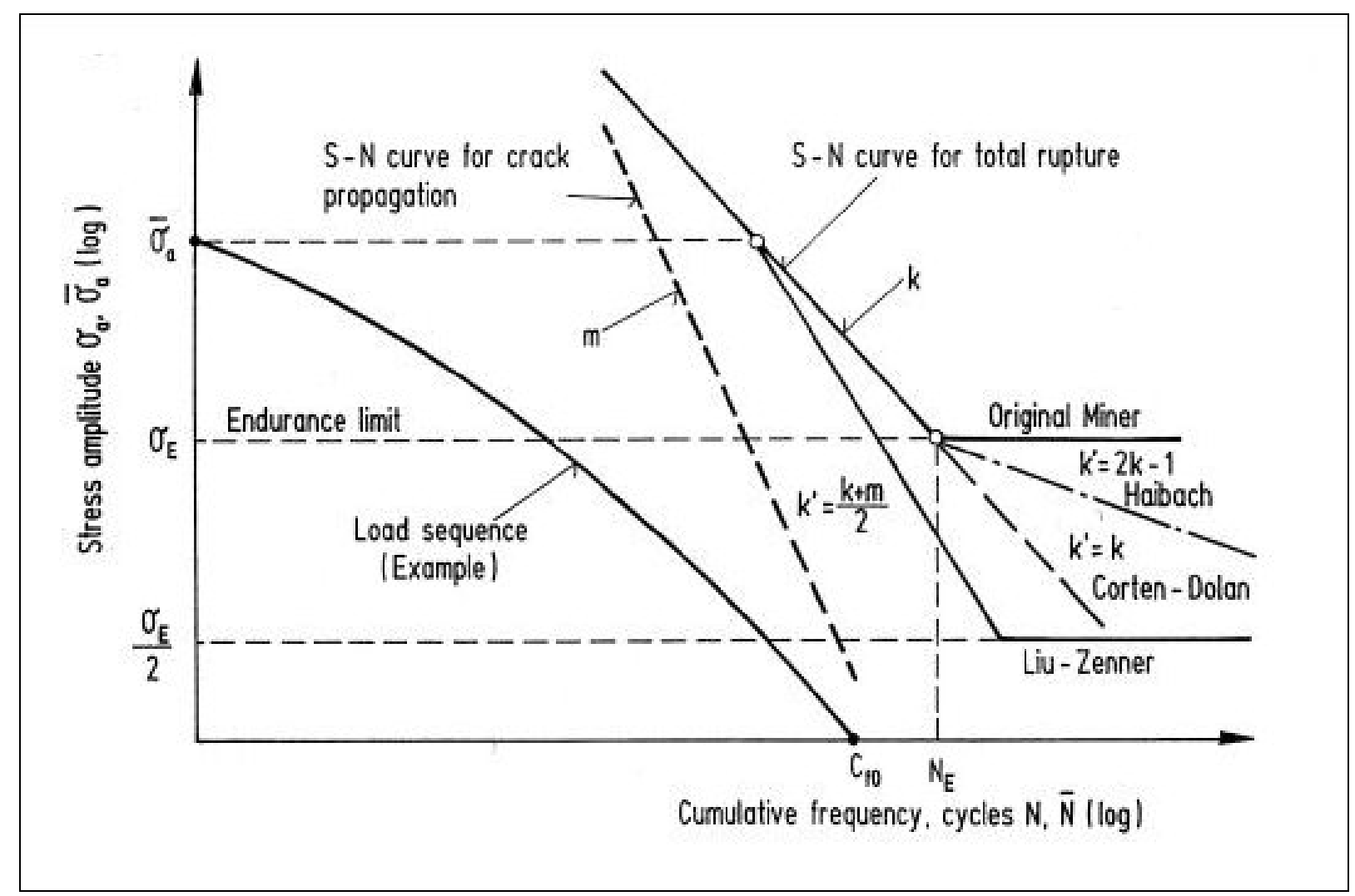

Bild 2.30: Ableitung der Wöhlerlinie für die Schadensakkumulation

Eulitz und Kotte haben eine umfangreiche Untersuchung über die Streuung der Abschätzung unter Belastung mit variablen Amplituden durchgeführt [Eul97-1, Eul97-2]. Dabei wurden verschiedene Werkstoffe (Stahl, Aluminium, Eisenguss) und Belastungsarten (Zug/Druck und Flachbiegung) getrennt nach vier unterschiedlichen Verfahren der Schadensrechnung erprobt, Bild 2.28. Es zeigt sich, dass die Streuung bei der Schadensrechnung nach Miner-Liu/Zenner insgesamt am kleinsten ausfällt. Für Aluminium schwankt die mittlere ermittelte Schadenssumme je nach Verfahren zwischen 0,24 für modifiziert Miner und 0,63 für Miner-Liu/Zenner. Bei der Untersuchung von Schweißverbindungen stellt sich heraus, dass die Streuung der Schadenssumme für Aluminium deutlich größer ist als für Stahl; die mittlere Schadenssumme liegt für Aluminiumschweißverbindungen mit 0,27 deutlich unter der für Stahl mit 0,45, Bild 2.28.

In den neusten Regelwerken [FKM03, Hob05] und aktuellen Veröffentlichungen [Son05-3] werden aufgrund der oben geschilderten Erkenntnisse folgende Empfehlungen gegeben: Für die Lebensdauerabschätzung von Schweißverbindungen, sowohl aus Stahl als auch aus Aluminium, wird mit einer tatsächlichen Schadenssumme von $D=0,5$ gegen eine Wöhlerlinie mit $k=3,0$ gerechnet. Die zugrunde gelegte Bemessungswöhlerlinie knickt bei $N=10^{7}$ Schwingspielen ab. Danach wird diese mit einer Neigung von $k^{\prime}=5,0$ weitergeführt, Bild 2.31. Darüber hinaus haben auch Mittellastschwankungen einen schädigenden Einfluß auf die Lebensdauer. Dieser wird durch eine empfohlene tatsächlichen Schadenssummen von $D=0,2$ berücksichtigt, was sowohl für Stahl als auch Aluminium gilt (Unterschied größer als 1:3 in der Schwingspielzahl beim Vergleich von Kollektiven nach der Überschreitungshäufigkeit und Bereichspaarzählung [Bux92]).

Andere Vorgehensweisen zur Lebensdauerabschätzung, wie sie z. B. im Eurocode [Eur92, Eur98] verankert sind, liefern ähnliche Ergebnisse [Son89]. 


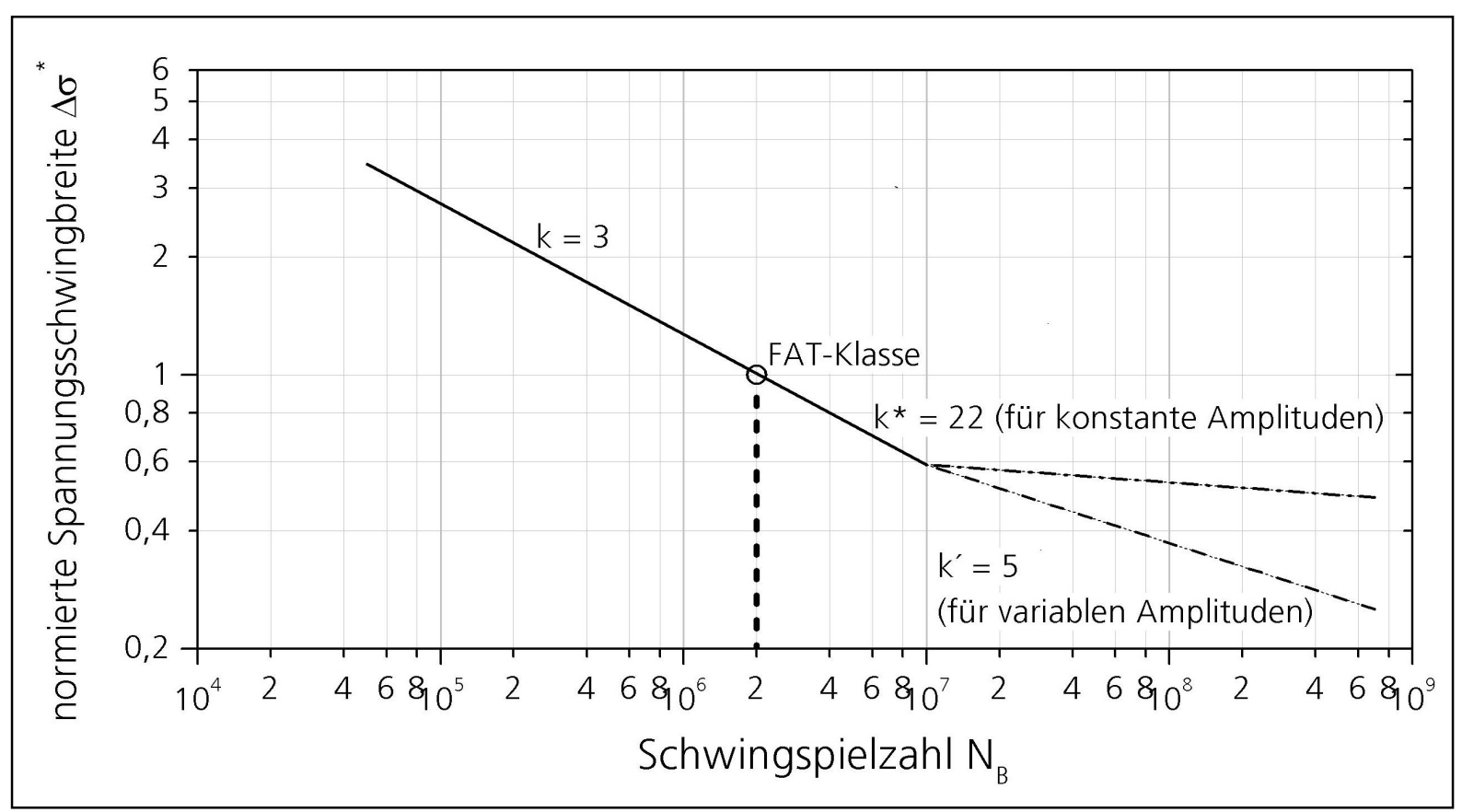

Bild 2.31: Normierte Wöhlerlinie für die Lebensdauerabschätzung nach IIW

Keines der dargestellten Konzepte oder Modelle führt für sich allein zum Erfolg. Es fehlt an gesicherten Erkenntnissen darüber, welche Berechnungsmodelle zu bevorzugen sind und welche Treffsicherheit mit innen erzielt werden kann. Um die Güte der Lebensdauerabschätzungen zu steigern, kann die Verknüpfung der verschiedenen Modelle erfolgreich sein. Es sei darauf hingewiesen, dass Werkstoffkennwerte ermittlungsbedingte Fehler beinhalten können, die unterschiedlich gravierende Auswirkungen auf die Abschätzung der Lebensdauer haben [Gra94]. Hierbei kommt es auch auf eine zutreffende Erfassung werkstoff- und fertigungsbedingter Streuungen an, die Grundlage für Sicherheitsbetrachtungen sind [Hai02, Hai89, Bux92].

Nach wie vor bestehen viele Unsicherheiten in der betriebsfesten Auslegung von Bauteilen, insbesondere bei Komponenten aus Aluminium. Dies ist zum einen auf die im Vergleich zu Stahl geringere Anzahl von Versuchen und Erfahrungen zurückzuführen, zum anderen auf die weniger eindeutige Bestimmung des Abknickpunktes der Wöhlerlinie und der so genannten Dauerfestigkeit. Mit Hilfe von Experimenten müssen deswegen weitere Erfahrungen gesammelt werden, um die verschiedenen Vorhersagekonzepte zu bestätigen oder besser zutreffende Konzepte, die weitere Parameter berücksichtigen, zu entwickeln. Bei Sicherheitsbauteilen, deren Versagen zu Schaden an Leib und Leben führen kann und der verantwortliche Hersteller mit staatsanwaltlichen Ermittlungen konfrontiert wird, darf kein Risiko eingegangen werden. Vor der Produktfreigabe kann auf die Durchführung eines Lebensdauernachweisversuches nicht verzichtet werden.

\subsection{Ansätze für die vorliegende Arbeit}

Auf den Erkenntnissen aus den vorgenannten Arbeiten aufbauend ergibt sich die Aufgabe, ein geeignetes Konzept für eine Bewertung des Festigkeitsverhaltens von Aluminiumschweißverbindungen unter konstanten Amplituden zur Verfügung zu stellen, welches Eingang in Regelwerke, wie z.B. den IIW-Empfehlungen [Hob03] oder den FKM-Richtlinien [FKM03] finden kann. 
Die Schwingfestigkeit der Schweißverbindungen wird durch die Geometrie (Spannungskonzentration), den Werkstoffzustand (Grundwerkstoff, Schweißgut, Wärmeeinflusszone), die Belastungshöhe und überlagerte Mittelspannungen beeinflusst. Da die Kenntnis dieser Einflussgrößen die Grundlage für die Entwicklung eines zutreffenden Bemessungskonzeptes darstellt, werden diese Größen durch Schwingfestigkeitsversuche mit konstanten Amplituden quantifiziert. Im einzelnen wurden folgende Versuche durchgeführt:

- Zunächst wurden Schwingfestigkeitsversuche am Grundwerkstoff, dem Schweißgut und der Wärmeeinflusszone der warmausgehärteten Legierung AlMgSi1 T6 (AW-6082 T6) mit ungekerbten und rissartig gekerbten Flachproben durchgeführt. Anhand dieser Untersuchung und mit den Ergebnisse der vorangegangenen Untersuchung [Wer01] zur naturharten Legierung AlMg4,5Mn (AW-5083) wurde die Ersatzstrukturlänge bestimmt, um das Konzept der Mikrostützwirkung bei Anwendung auf warmausgehärtete und naturharte Aluminiumlegierungen zu überprüfen.

- Es wurde das Schwingfestigkeitsverhalten von Aluminiumschweißverbindungen der Legierung AlMgSi1 T6 (AW-6082 T6) verschiedener Kerbschärfen im Einstufenversuch unter Wechselund Schwellbeanspruchung untersucht. Das Schwingfestigkeitsverhalten dieser Legierung wurde zusammen mit Ergebnissen der Legierung AlMg4,5Mn (AW-5083) bewertet.

- Ebenfalls wurden metallografische Untersuchungen zur Charakterisierung des Gefüges und des Vergleiches der Legierungstypen vorgenommen.

- An ungekerbten Flachproben wurden die zügigen und zyklischen Spannungs-DehnungsKurven sowie die dehnungs- und lastgesteuert aufgenommenen Anrisswöhlerlinien für Grundwerkstoff, Schweißgut und Wärmeeinflusszone ermittelt. Eine Gegenüberstellung mit den Werten der Legierung AlMg4,5Mn (AW-5083) wurde durchgeführt.

- Die bruchmechanischen Kennwerte für Grundwerkstoff, Schweißgut und Wärmeeinflusszone wurden aus Rissfortschrittsuntersuchungen mit den rissartig gekerbten Flachproben ermittelt. Zur Charakterisierung des Rissschließverhaltens wurden die Untersuchungen unter Wechselund Schwellbeanspruchungen durchgeführt. Auch hier erfolgte eine Gegenüberstellung mit den Ergebnissen der Legierung AlMg4,5Mn (AW-5083).

Die beiden letztgenannten Versuchsprogramme zur Ermittlung der zyklischen SpannungsDehungskurven bzw. der Dehnungswöhlerlinie sowie die bruchmechanischen Versuche dienen der umfassenden Werkstoffcharakterisierung, werden aber bei der Entwicklung der Berechnungskonzepte für Schweißverbindungen im Rahmen dieser Arbeit nicht verwendet.

Zunächst wurde das Konzept der Mikrostützwirkung dahingehend überprüft, ob es in der Lage ist, das Verhalten unterschiedlicher Aluminiumlegierungstypen - warmaushärtbare und naturharte - zu berücksichtigen.

Danach wurde das Konzept der Mikrostützwirkung soweit abstrahiert, dass versucht wurde, einen rein fiktiven Ersatzradius zu finden, der unabhängig vom Gefügezustand der betrachteten Legierung ist. Mit der Anwendung dieses Konzeptes des fiktiven Ersatzradius auf die hier ermittelten Ergebnisse und auf weitere Versuchsergebnisse aus anderen Untersuchungen zur Lebensdauerabschätzung von Schweißverbindungen aus Aluminium wurde eine regelwerkfähige Aufbereitung wie bei Stahl für diese Konzepte erarbeitet. 


\section{Werkstoffe, Proben und Versuchsdurchführung}

\subsection{Werkstoffe und Proben}

Für die Untersuchung wurde die warmausgehärtete Aluminiumknetlegierung AlMgSi1 T6 (AW6082 T6) verwendet. Der Werkstoff wurde als Platten in der Stärke $5 \mathrm{~mm}$ von Hoogovens Aluminium Produkte $\mathrm{GmbH}$, Koblenz, geliefert. Der Vergleichswerkstoff ist eine naturharte Aluminiumknetlegierung AlMg4,5Mn (AW-5083) in der Stärke $5 \mathrm{~mm}$, ebenfalls von Hoogovens Aluminium Produkte $\mathrm{GmbH}$, Koblenz, geliefert [Wer01]. Aus einer weiteren Untersuchung wurden Versuchsergebnisse mit Schweißverbindungen der Legierungen AlMgSi0,7 T6 (AW6005A T6) und AlMg4,5Mn0,7 (AW-5083) in einige Auswertungen mit einbezogen [Grz03, Son02]. Die chemischen Zusammensetzungen und die mechanischen Werkstoffkennwerte sind in Tabelle 3.1 und Tabelle 3.2 zusammengefasst.

\begin{tabular}{|l|l|c|c|c|c|c|c|c|}
\hline Werkstoff & & Si & Fe & Cu & Mn & Mg & Cr & Zn \\
\hline AlMgSi1 (AW-6082) & warmaushärtbar & 0,87 & 0,24 & 0,05 & 0,65 & 0,77 & 0,02 & 0,01 \\
\hline AlMgSi0,7 (AW-6005A) & warmaushärtbar & 0,57 & 0,16 & 0,11 & 0,10 & 0,64 & 0,06 & 0,01 \\
\hline AlMg4,5Mn (AW-5083), Charge A & naturhart & 0,30 & 0,23 & 0,09 & 0,63 & 4,65 & 0,09 & 0,13 \\
\hline AlMg4,5Mn0,7 (AW-5083), Charge B & naturhart & 0,13 & 0,22 & 0,03 & 0,66 & 4,42 & 0,10 & 0,01 \\
\hline
\end{tabular}

Tabelle 3.1: Chemische Zusammensetzung der Legierungen

\begin{tabular}{|l|l|c|c|c|c|}
\hline Werkstoff & Wärmebehandlung & $\begin{array}{c}\text { Dicke d } \\
{[\mathbf{m m}]}\end{array}$ & $\begin{array}{c}\mathbf{R}_{\mathbf{p 0 , 2}} \\
{[\mathbf{M P a}]}\end{array}$ & $\begin{array}{c}\mathbf{R}_{\mathbf{m}} \\
{[\mathbf{M P a}]}\end{array}$ & $\begin{array}{c}\mathbf{A}_{\mathbf{5}} \\
{[\%]}\end{array}$ \\
\hline AlMgSi1 T6 (AW-6082 T6) & 8h bei $185^{\circ} \mathrm{C}$ & 5 & 287 & 318 & 13,4 \\
\hline AlMgSi0,7 T6 (AW-6005A T6) & 8h bei $185^{\circ} \mathrm{C}$ & 5 & 254 & 285 & $\mathrm{k} . \mathrm{A}$. \\
\hline AlMg4,5Mn (AW-5083), Charge A & naturharte Legierung & 5 & 175 & 303 & 20,2 \\
\hline AlMg4,5Mn0,7 (AW-5083), Charge B & naturharte Legierung & 5 & 166 & 253 & 25,5 \\
\hline
\end{tabular}

Tabelle 3.2: Mechanische Kennwerte der Legierungen 


\subsubsection{Probenform und Fertigung}

Zur Ermittlung des Schwingfestigkeitsverhaltens für den Grundwerkstoff (GW) (Bild 3.1 und Bild 3.4), das Schweißgut (SG) (Bild 3.2 und Bild 3.5) und die Wärmeeinflusszone (WEZ) (Bild 3.3 und Bild 3.6) wurden sechs verschiedene Probenformen eingesetzt, jeweils ungekerbt und rissartig gekerbt. Für die Schwingfestigkeitsversuche mit dem Schweißgut wurden geschweißte Stumpfstoßproben verwendet, deren Schweißraupe beidseitig abgearbeitet wurde, so dass sie im versagenskritischen Bereich nur das Schweißgut ohne Nahtüberhöhung und Nahtwurzelkerbe aufweisen.

\begin{tabular}{|l|l|l|l|}
\hline Werkstoffzustand & Probenform & Kerbradius $\rho[\mathrm{mm}]$ & Formzahl $\mathrm{K}_{\mathrm{t}, \mathrm{ax}}$ \\
\hline GW, SG, WEZ & ungekerbte Flachproben $20 \times 5 \mathrm{~mm}^{2}$ & 50 & 1,0 \\
\hline GW, SG, WEZ & $\begin{array}{l}\text { rissartig gekerbte Flachproben } 40 \times 5 \mathrm{~mm}^{2} \\
\text { (Rissfortschrittsprobe) }\end{array}$ & $0,07^{1)} / 0,15^{2)}$ & $11,2^{1)} / 10,2^{2)}$ \\
\hline
\end{tabular}

1) Werkstoff AlMgSi1 T6 (AW-6082)

2) Werkstoff AlMg4,5Mn (AW-5083)

Die rissartigen Kerben der Proben wurden durch einen Sägeschnitt erzeugt. Durch die geringfügig unterschiedliche Geometrie der verwendeten Sägeblätter ergaben sich für beide Werkstoffe unterschiedliche Kerbradien und Kerbformen und damit unterschiedliche Kerbformzahlen. Dies erklärt auch den Unterschied zwischen den Zeichnungsangaben und den an den realen Proben gemessenen Geometrien. Die für die weiteren Untersuchungen verwendeten und oben aufgelisteten Geometriewerte entstammen gemittelten Messungen an Makroaufnahmen mehrere Proben.

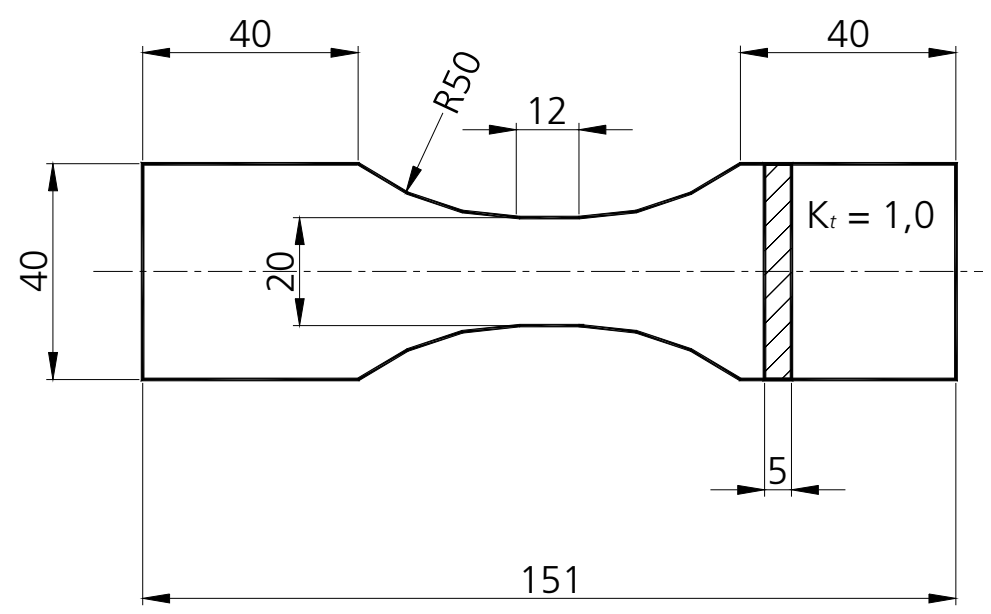

Bild 3.1: Geometrie Basisprobe - Grundwerkstoff ungekerbt 


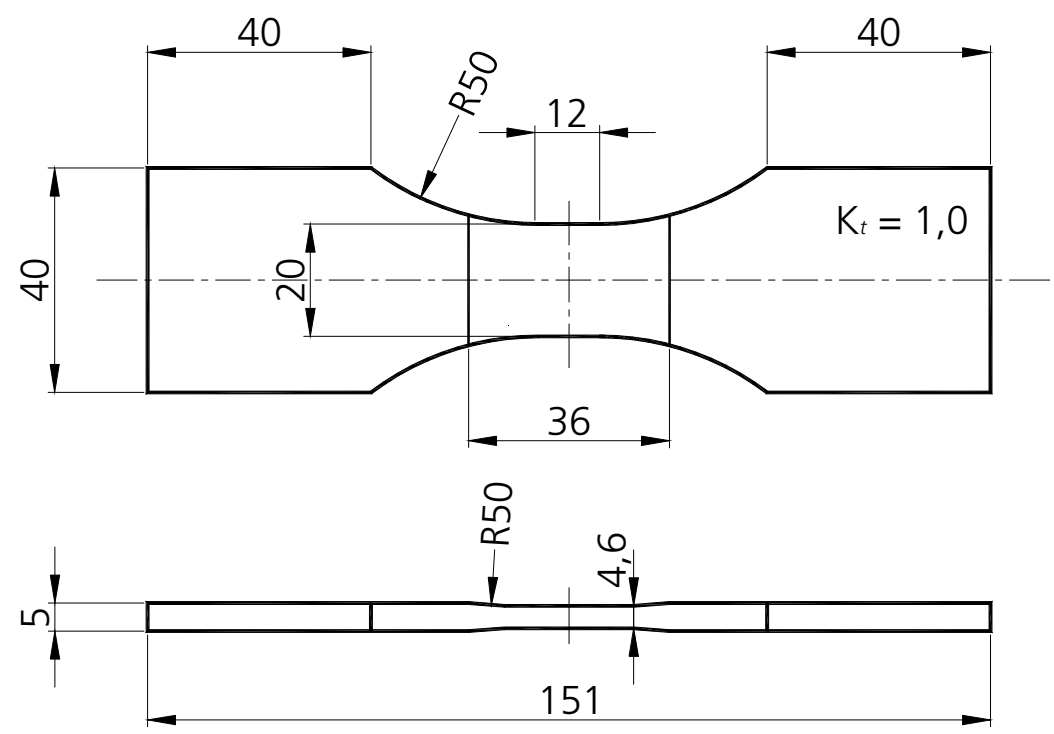

Bild 3.2: Geometrie Basisprobe - Schweißgut ungekerbt

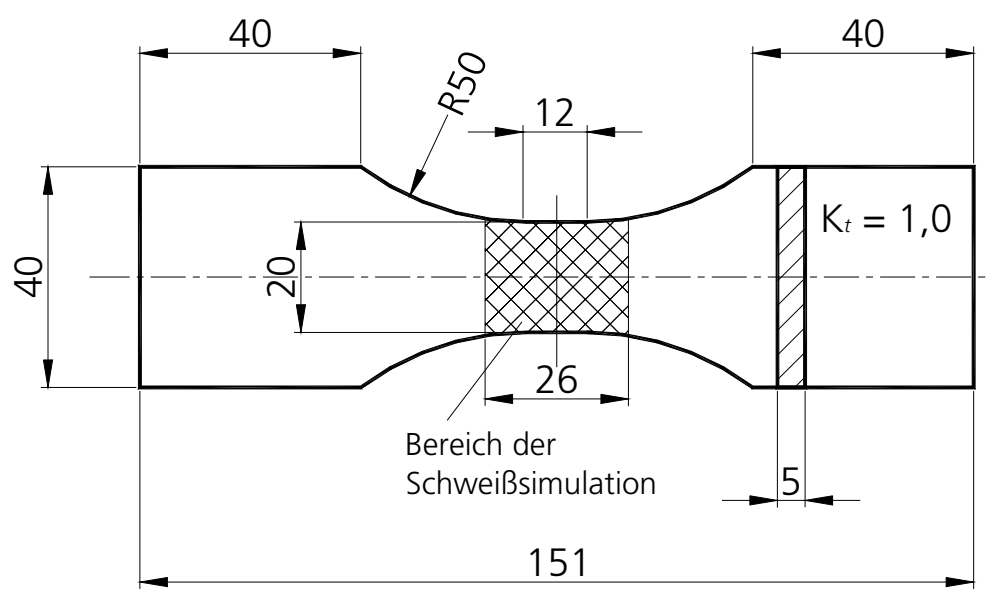

Bild 3.3: Geometrie Basisprobe - Wärmeeinflusszone ungekerbt 

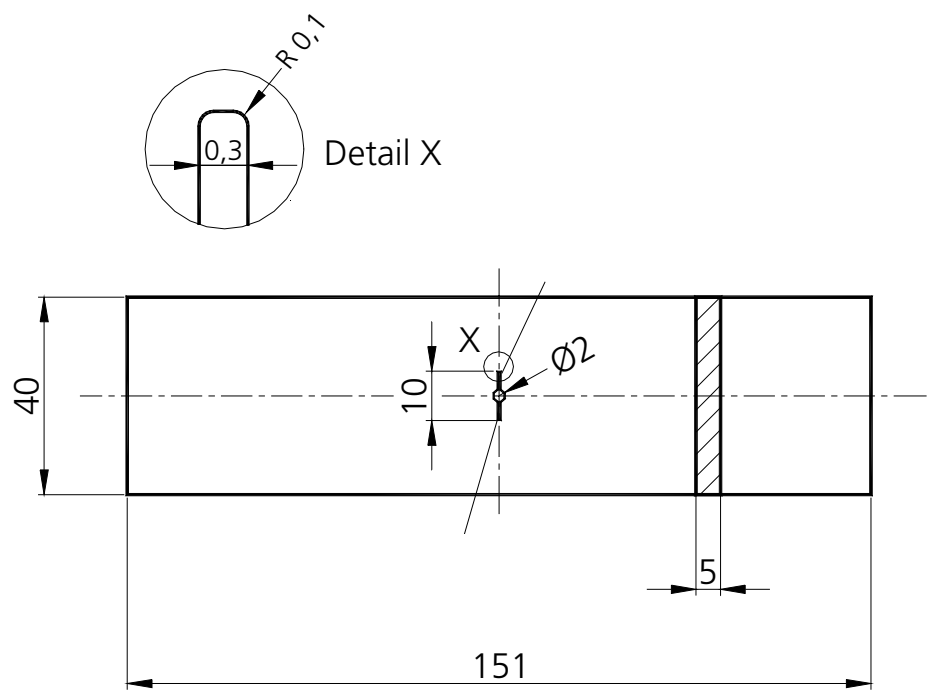

Bild 3.4: Geometrie Basisprobe - Grundwerkstoff scharf gekerbt
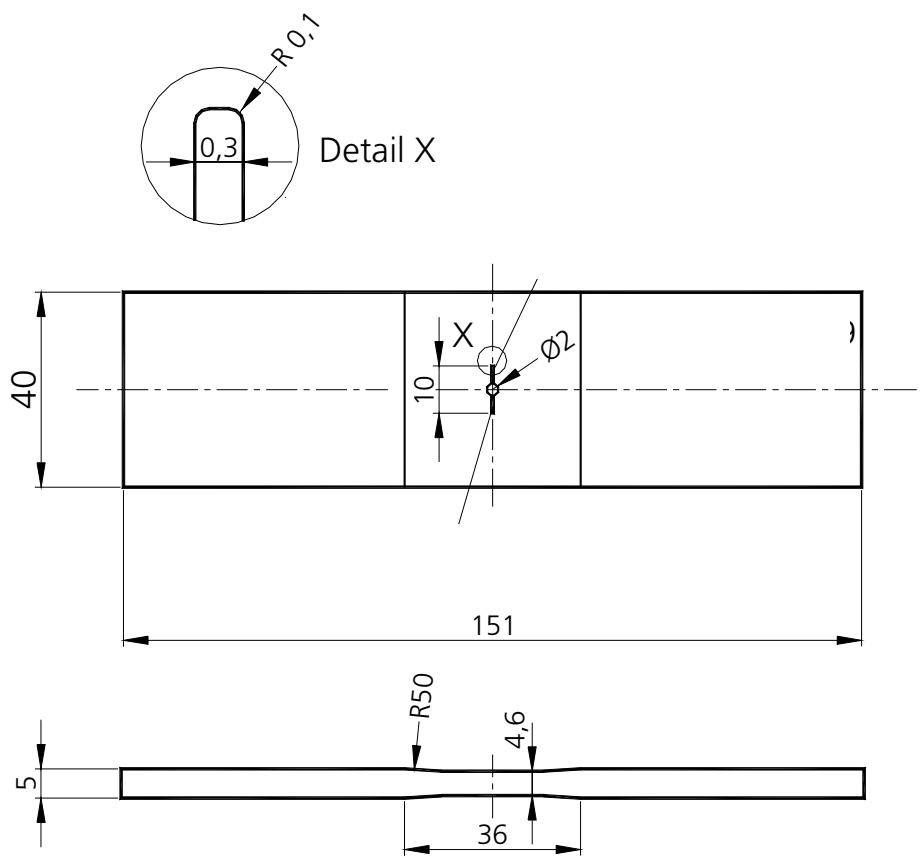

Bild 3.5: Geometrie Basisprobe - Schweissgut scharf gekerbt 


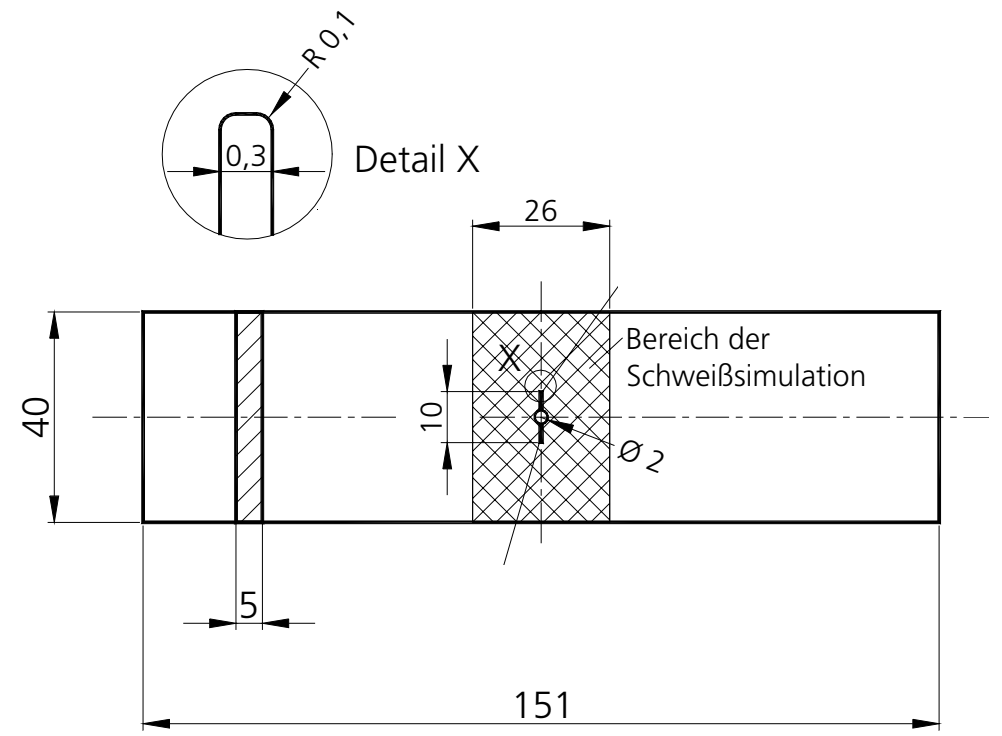

Bild 3.6: Geometrie Basisprobe - Wärmeeinflusszone scharf gekerbt

Die Formzahlen $\mathrm{K}_{\mathrm{t}, \mathrm{a}}$ der gekerbten Flachproben wurden rechnerisch mittels der Finite-ElementMethode (Programm Patran) und nach Peterson und Neuber ermittelt [Pet74, Neu85].

Weiterhin wurden Schwingfestigkeitsversuche mit geschweißten Verbindungen unterschiedlicher Geometrie, Bild 3.7 bis Bild 3.9, vorgenommen: 

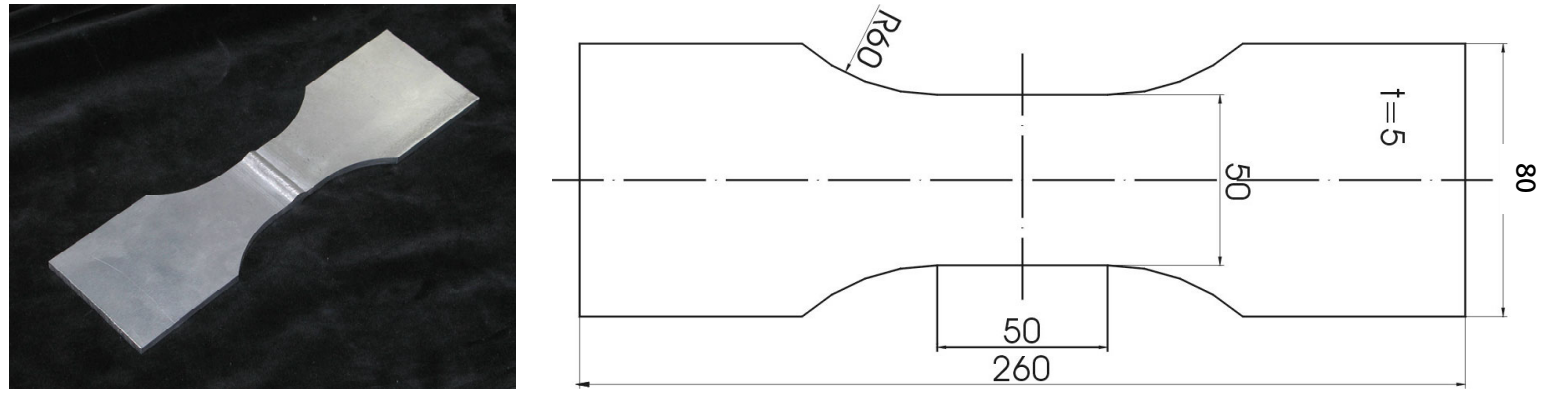

Bild 3.7: Stumpfstoß ohne Wurzelspalt; $r=1,5 \mathrm{~mm}, K_{t}=1,50$ (Geometrie und $K_{t}$-Werte nur für AlMgSi1 T6)
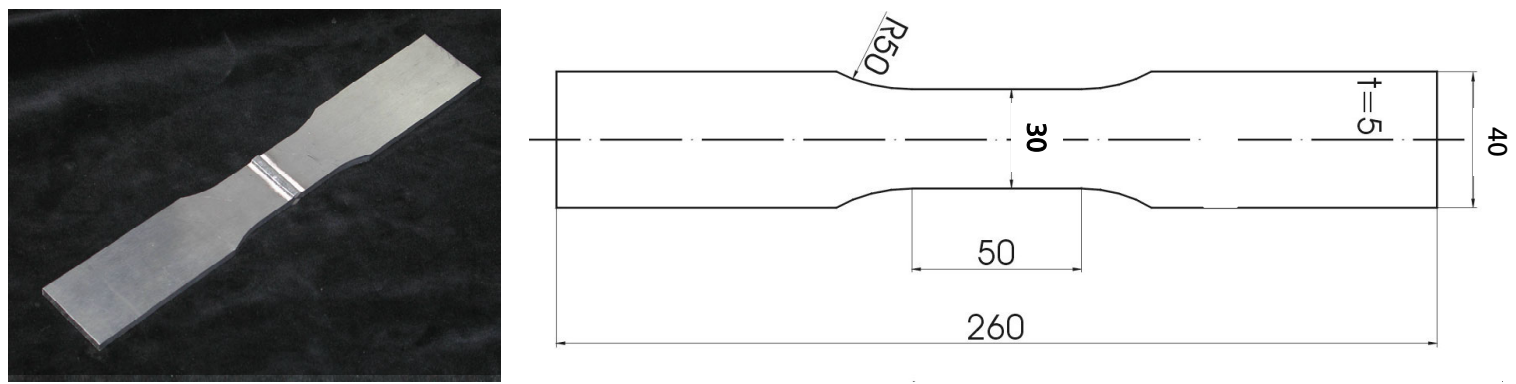

Bild 3.8: Stumpfstoß mit Wurzelspalt; Spalt: 1,7 - 2,2 mm, $r=0,1 \mathrm{~mm}, \mathrm{~K}_{\mathrm{t}, \max }=7,45$ (Geometrie und $\mathrm{K}_{\mathrm{t}}$-Werte nur für AlMgSi1 T6)
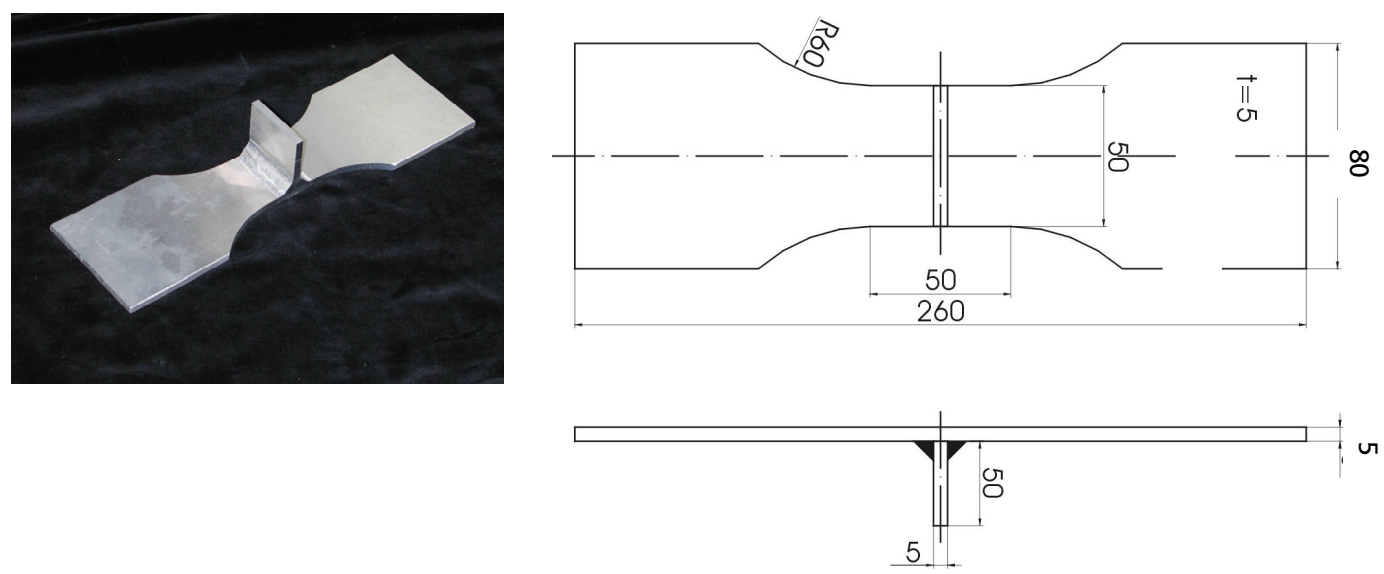

Bild 3.9: Quersteife; $r=1,2 \mathrm{~mm}, \mathrm{~K}_{\mathrm{t}}=1,36$ (Geometrie und $\mathrm{K}_{\mathrm{t}}$-Werte nur für AIMgSi1 T6) 


\section{Schweißnaht}

ohne Wurzelspalt

ohne Wurzelspalt

mit Wurzelspalt

mit Wurzelspalt

Quersteife

\section{Werkstoff}

AlMgSi1 T6

AlMg4,5Mn

AlMgSi1 T6

AlMg4,5Mn

AlMgSi1 T6
Dicke

t [mm]

5,0

5,0

5,0

5,0

5,0
Kerbradius $\rho$ [mm]

1,50

2,00

0,10

0,10

1,20
Formzahl

$\mathrm{K}_{\mathrm{t}, \mathrm{a}}$

1,50

1,40

7,45

6,72

1,36

Die Berechnung der Formzahlen der Schweißproben ist in Abschnitt 3.1.4 erläutert. Aus den unterschiedlichen geometrischen Ausbildungen der Nahtüberhöhung, der Nahtübergangskerben und der Nahtwurzelkerben ergibt sich, dass gleiche Kerbradien zu unterschiedlichen Kerbformzahlen bei verschiedenen Schweißverbindungen führen. Die ungeschweißten und geschweißten Proben wurden aus $5 \mathrm{~mm}$ dicken Aluminiumblechen entnommen, wobei die Walzrichtung quer zur späteren Belastungsrichtung lag.

Die Schweißung der Legierung AlMgSi1 T6 (AW-6082 T6) als Stumpfstoß mit und ohne Wurzelspalt und Quersteife erfolgte am Institut für Schweißtechnik (ifs) der Universität Braunschweig mit dem mechanisierten Metall-Inert-Gas-Schweißverfahren (MIG). Die Schweißungen der naturharten Vergleichslegierung AIMg4,5Mn (AW-5083) wurden von den Vereinigten Aluminium Werken AG (VAW), Bonn, ebenfalls mit dem MIG-Verfahren durchgeführt, um eine einheitliche Nahtgeometrie zu gewährleisten. Als Schweißzusatz wurde AlSi5 bei der Schweißung des AIMgSi1 T6 Werkstoffes und SG-AIMg4,5Mn bei den AlMg4,5MnVerbindungen eingesetzt. Die Schweißparameter sind in den Schweißprotokollen zusammengefasst, (siehe Anhang A: Schweißprotokolle).

\subsubsection{Härtemessungen}

An Schweißverbindungen wurden zur Gefügedokumentation lineare Kleinlasthärtemessungen (HV5) und flächige Mikrohärtemessungen (HV0,5) vorgenommen.

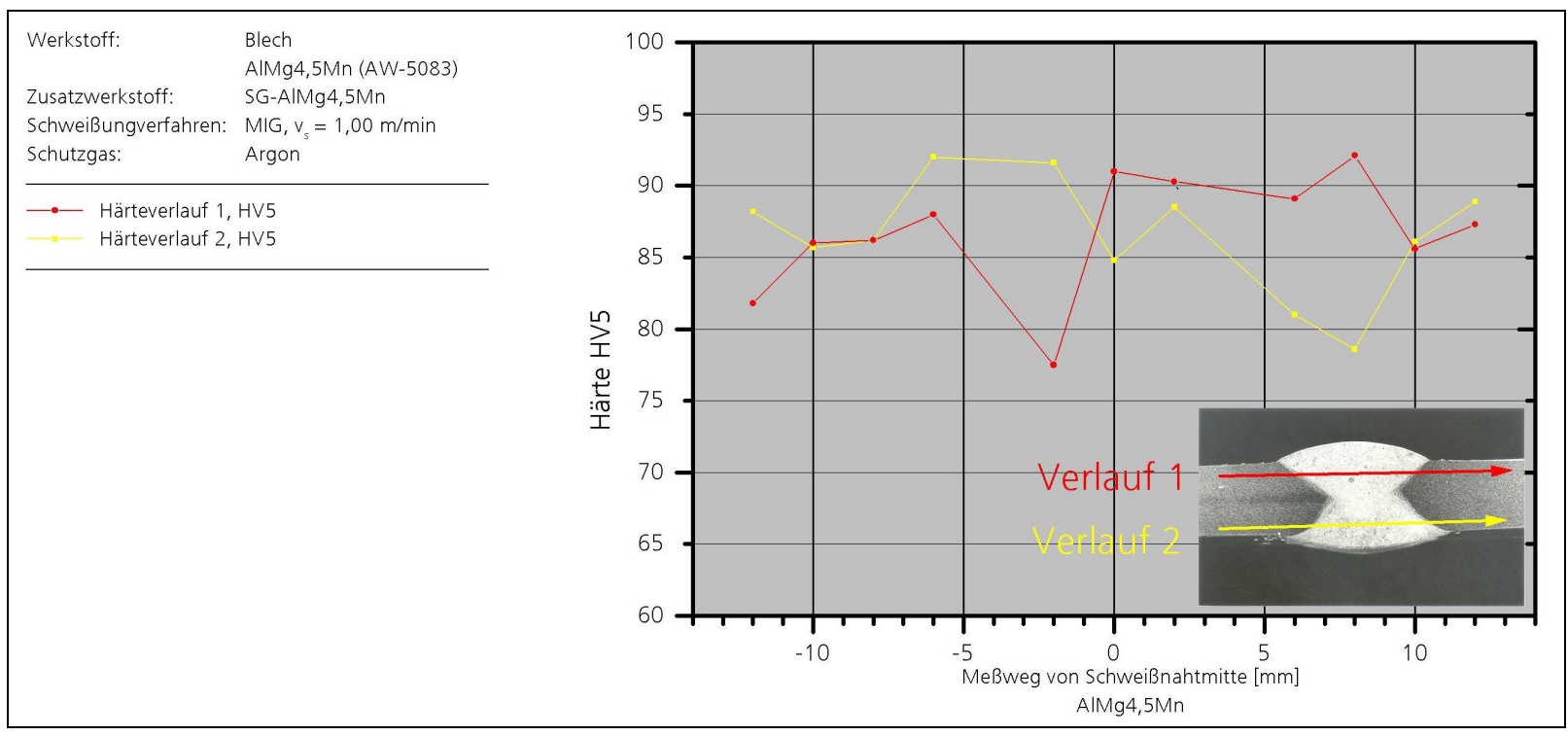

Bild 3.10: Lineare Härteverteilung über der Schweißnaht - Stumpfstoß AIMg4,5Mn 
An den linearen Härteverläufen erkennt man, im Rahmen der Streuung, den homogenen Verlauf der Härte HV5 über Grundwerkstoff - Wärmeeinflusszone - Schweißgut - Wärmeeinflusszone Grundwerkstoff bei der Legierung AlMg4,5Mn (AW-5083), Bild 3.10 und Bild 3.11. Diese beträgt im Mittel 85 HV5. Bei der Legierung AlMgSi1 T6 ist dagegen ein ausgeprägter inhomogener Verlauf über die Zone der Schweißnaht zu erkennen. Während im Grundwerkstoff im Mittel Werte von 100 bis 110 HV5 erreicht werden, fällt die Härte in der Wärmeeinflusszone und der Schweißnaht auf 70 HV5 ab. An der Schmelzlinie zwischen Wärmeeinflusszone und Schweißgut kann eine Aufhärtung bis zur Grundwerkstoffhärte beobachtet werden, Bild 3.11 bis Bild 3.13.

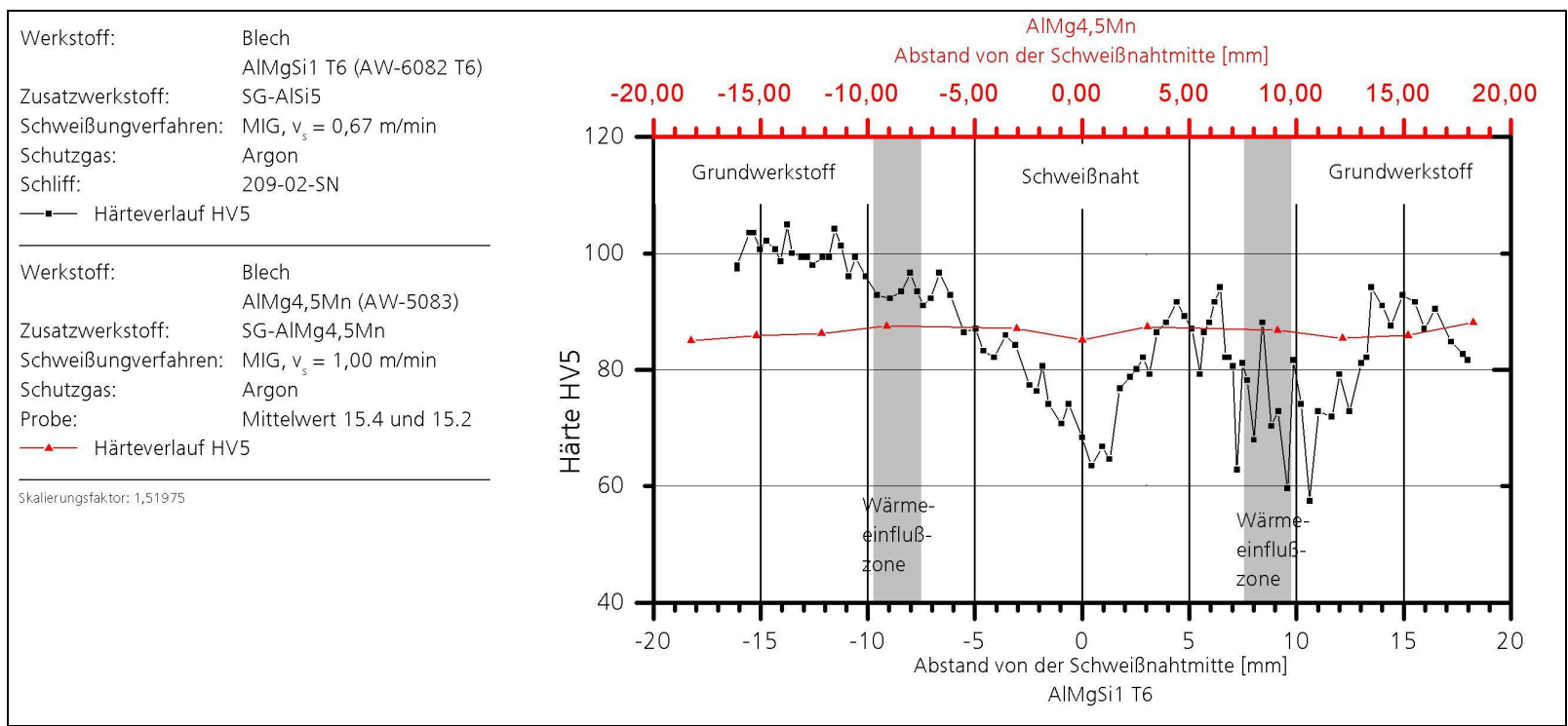

Bild 3.11: Lineare Härteverteilung über der Schweißnaht Vergleich

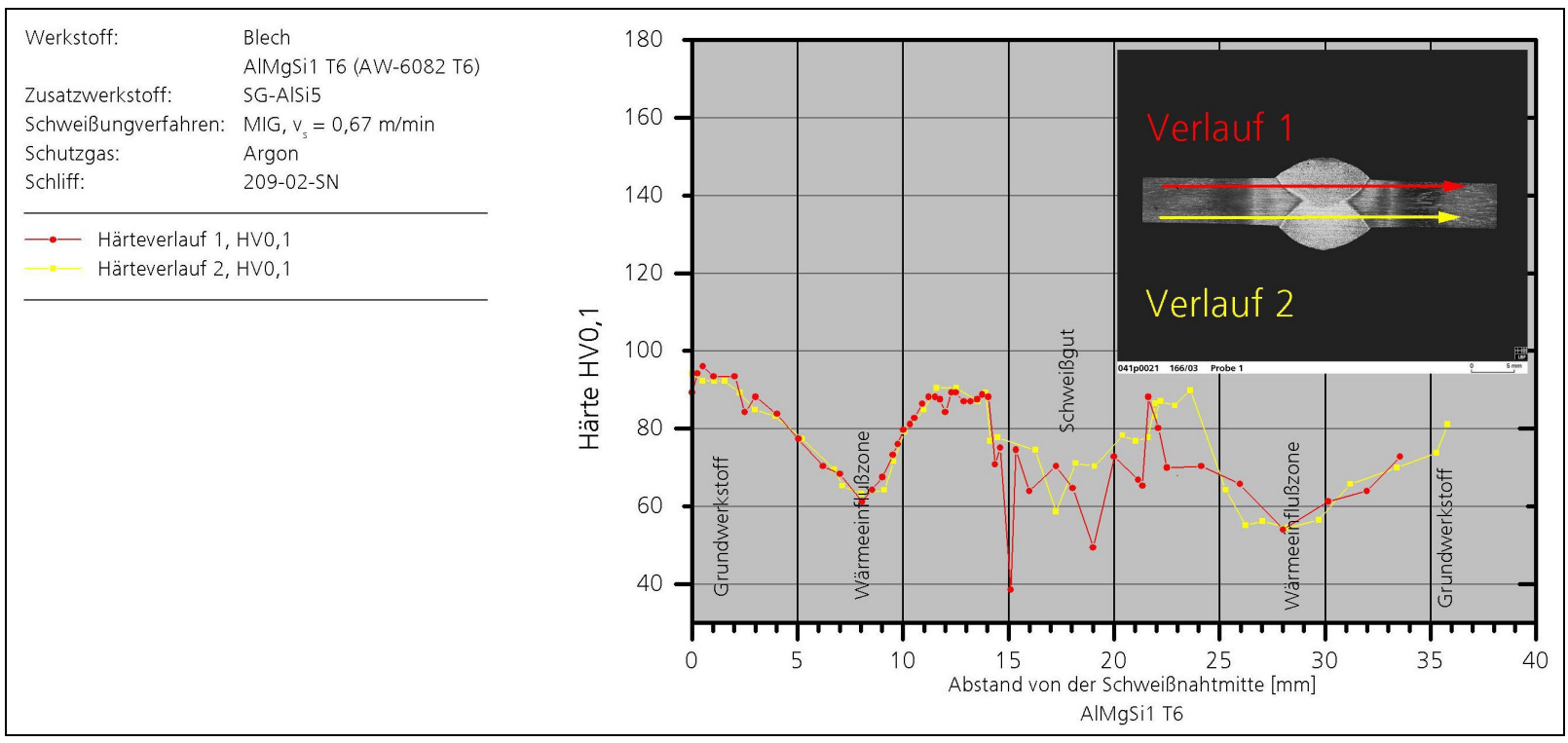

Bild 3.12: Lineare Härteverteilung über der Schweißnaht - Stumpfstoß AlMgSi1 T6 


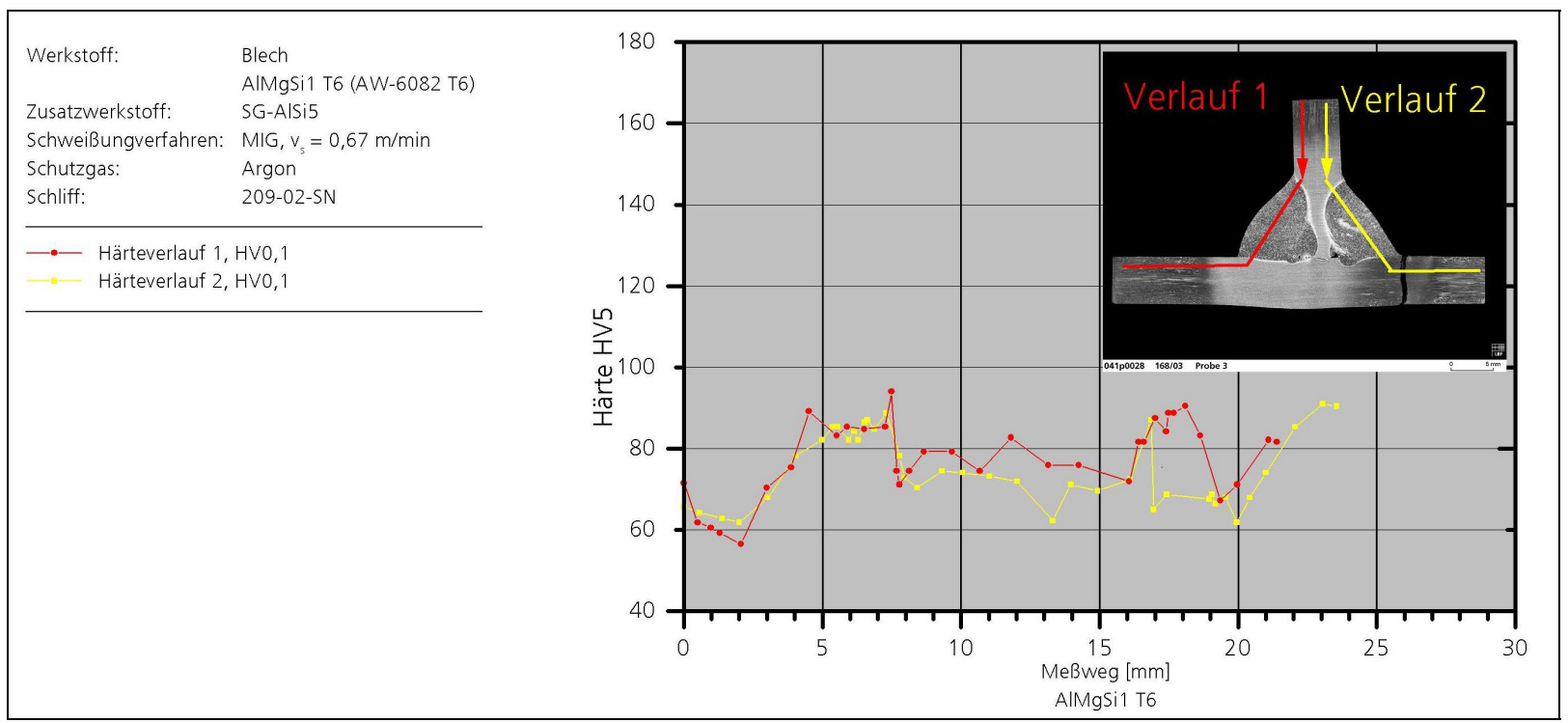

Bild 3.13: Lineare Härteverteilung über der Schweißnaht - Quersteife AIMgSi1 T6

Die flächige Mikrohärteverteilungen HV0,5 wurden am Institut für Schweißtechnik der Technischen Universität Braunschweig ermittelt. An den Messungen zeigt sich, wie oben beschrieben, die deutlich homogenere Härteverteilung bei der Legierung AlMg4,5Mn (AW-5083) gegenüber der Legierung AlMgSi1 T6 (AW-6082 T6).

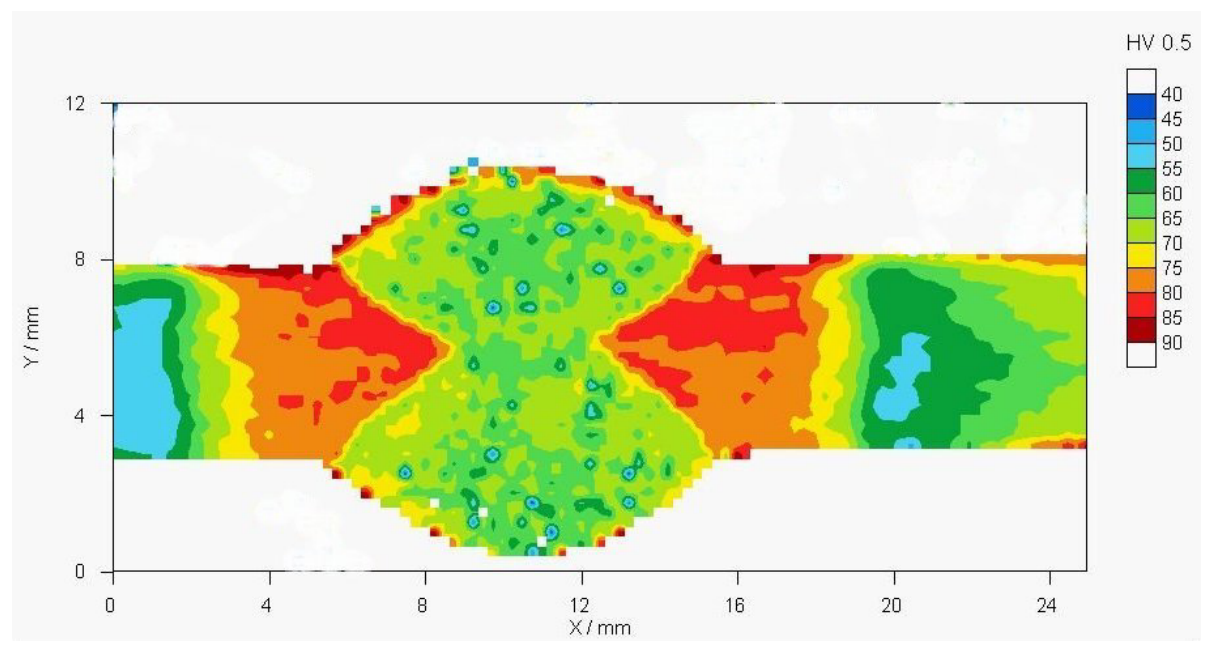

Bild 3.14: Flächige Härteverteilung - Stumpfstoß ohne Wurzelspalt AIMgSi1 T6 (AW-6082 T6) 


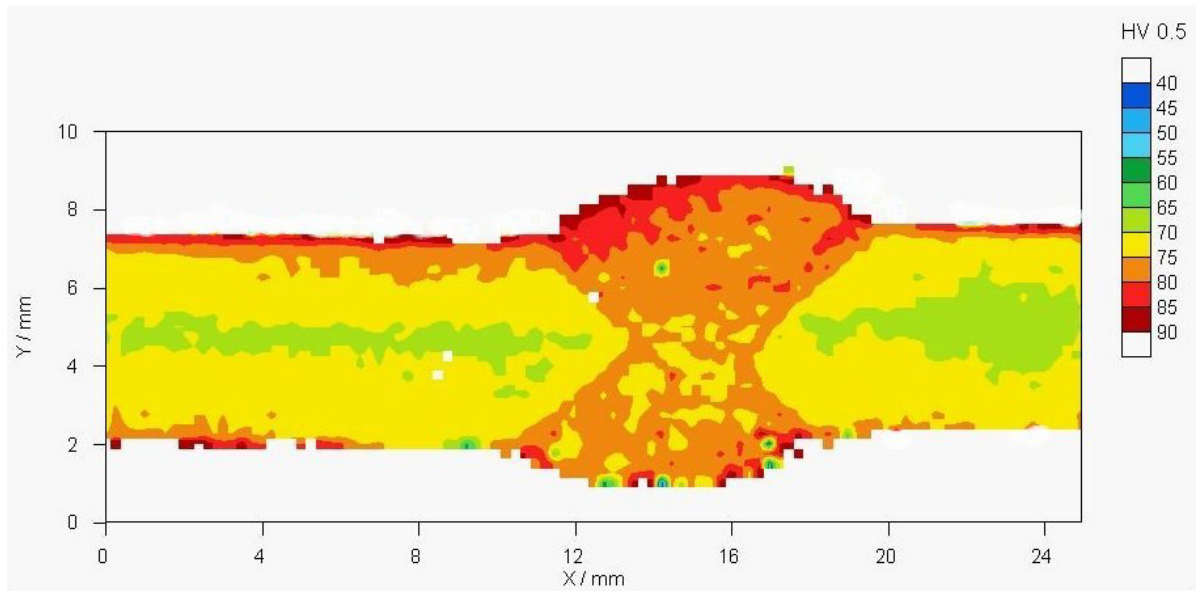

Bild 3.15: Flächige Härteverteilung - Stumpfstoß ohne Wurzelspalt AIMg4,5Mn (AW-5083)

Bei der Legierung AlMgSi1 T6 (AW-6082 T6) ergeben sich auch bei der Mikrohärtemessung Werte von $110 \mathrm{HV0,5}$. Die Härte des Schweißgutes beträgt in allen Fällen der Schweißnahtausbildung 60-80 HV0,5. Im Bereich der Wärmeeinflusszone, unmittelbar neben der Naht, hat während der Schweißung eine vollständige Rücklösung der festigkeitssteigernden MgSiAusscheidungen ( $\beta^{\prime \prime}$-Phase) stattgefunden. Nach entsprechender Auslagerungszeit steigt die Härte dort wieder auf Werte von ca. 80 HV0,5 an. In weiter von der Naht entfernten Bereichen der Wärmeeinflusszone hat aufgrund der etwas niedrigeren Temperaturen nur eine teilweise Rücklösung stattgefunden. Dies führt zu einer verstärkten Ausscheidung von nicht festigkeitssteigernden $\mathrm{Mg}_{2}$ Si-Ausscheidungen ( $\beta^{\prime}$-Phase) während des Schweißtemperaturzyklus.

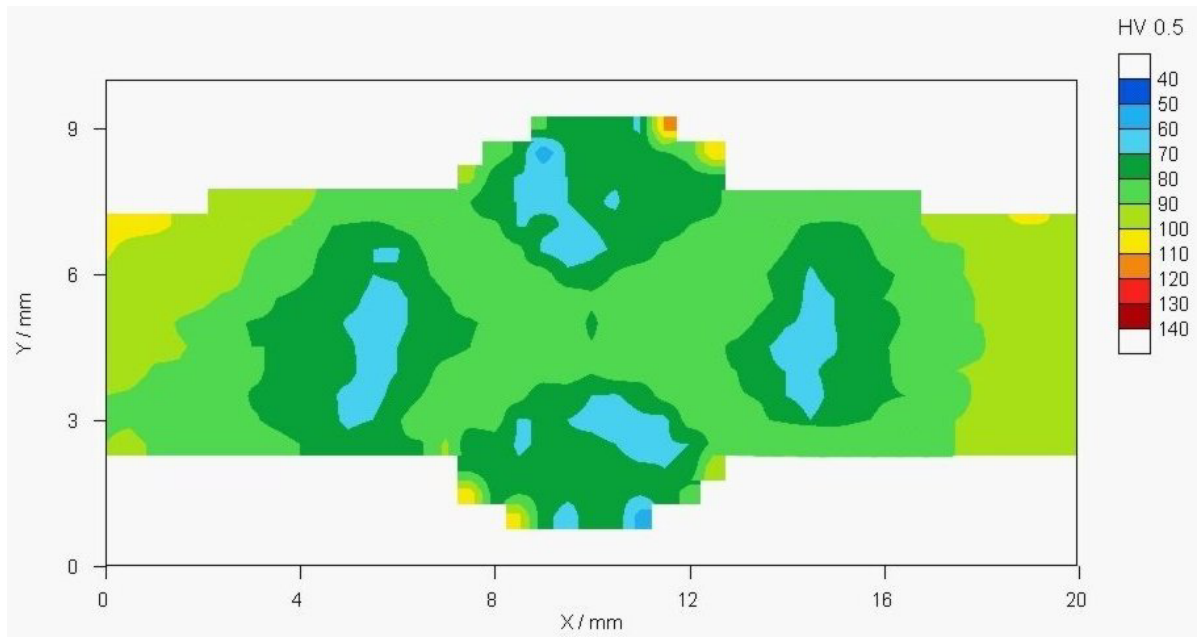

Bild 3.16: Flächige Härteverteilung - Stumpfstoß mit Wurzelspalt AIMgSi1 T6 (AW-6082 T6) 


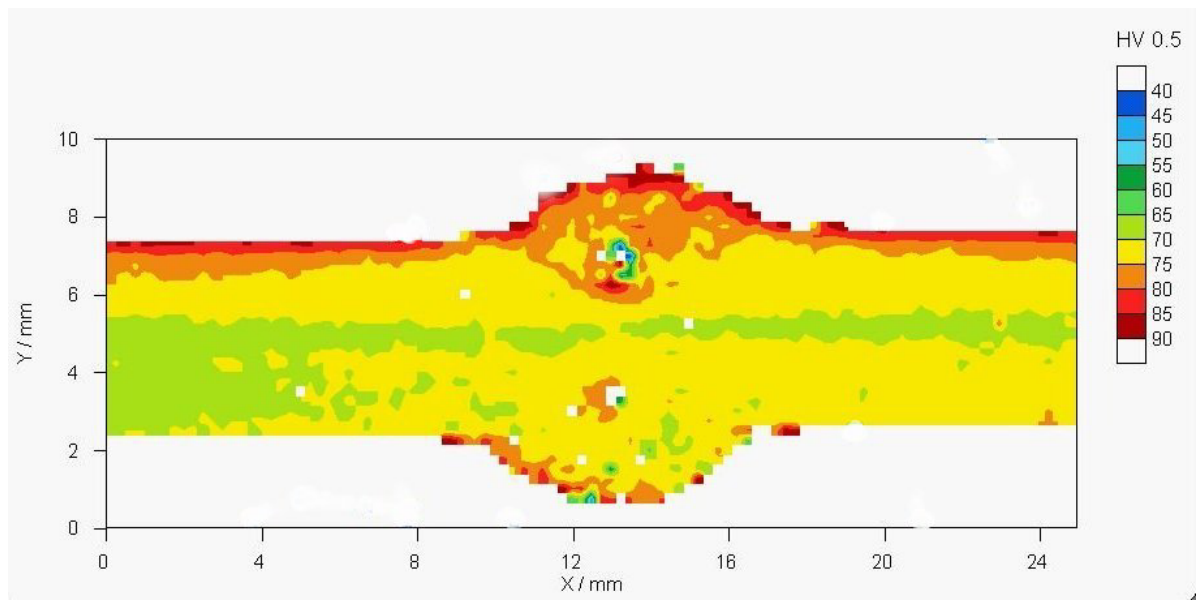

Bild 3.17: Flächige Härteverteilung - Stumpfstoß mit Wurzelspalt AlMg4,5Mn (AW-5083)

Die resultierende Härte in diesen Bereichen beträgt ca. 60 HV0,5. Aufgrund der im Vergleich zu den Schweißproben mit Wurzelspalt deutlich höheren Wärmeeinbringung bei der DV-Naht ohne Wurzelspalt und der Quersteife, Bild 3.14 und Bild 3.18, ist der Bereich der maximalen Rückhärtung $(80 \mathrm{HV} 0,5)$ hier sehr viel breiter. In der Härteverteilung der Schweißproben mit Wurzelspalt, Bild 3.16, ist der Bereich in dem keine maximalen Rückhärtung erreicht wurde, zu erkennen (blaue Bereiche in der WEZ).

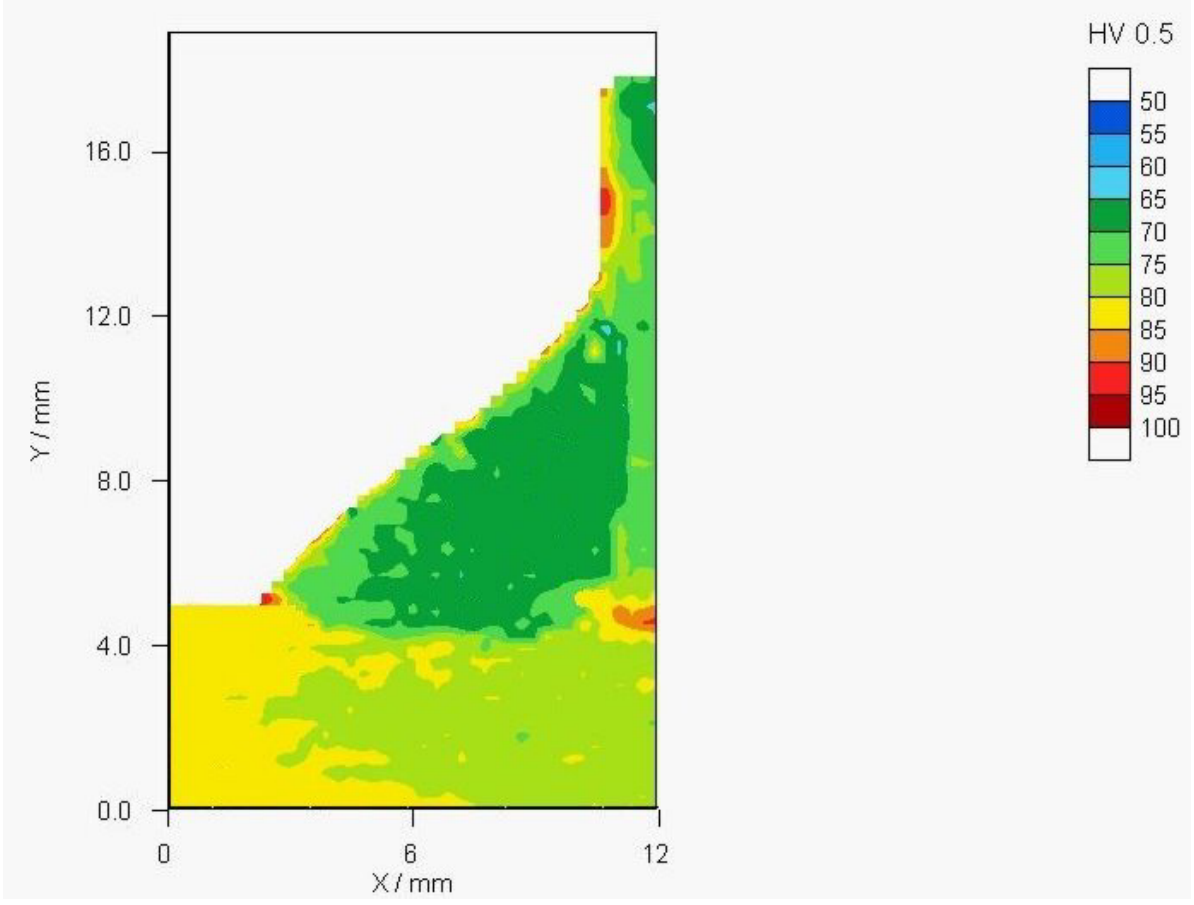

Bild 3.18: Flächige Härteverteilung - Quersteife AIMgSi1 T6 (AW-6082 T6) 